\title{
Best Estimate Radiation Flux Value-Added Procedure: Algorithm Operational Details and Explanations
}

October 2002

Y. Shi and C. N. Long

DOE, ARM, Pacific Northwest National Laboratory

Richland, Washington

Work supported by the U.S. Department of Energy,

Office of Energy Research, Office of Health and Environmental Research 


\section{Contents}

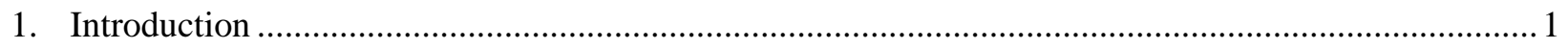

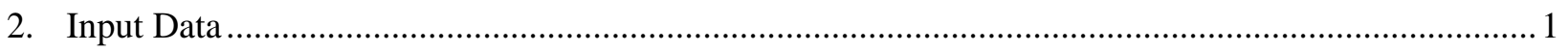

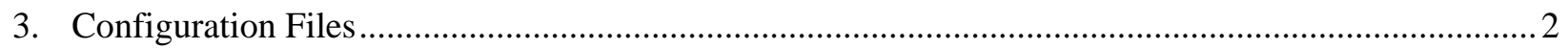

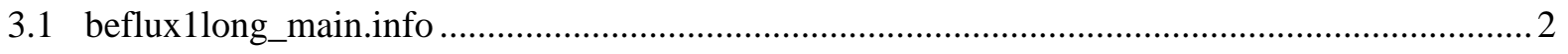

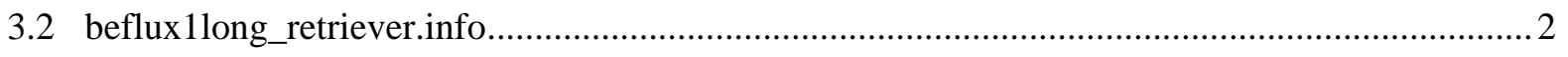

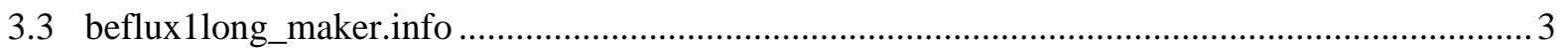

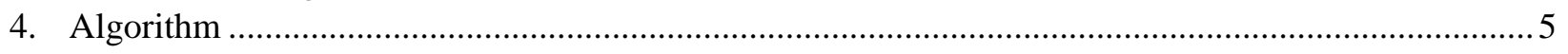

5. Criteria/Limits Used in Best Estimate Calculation...................................................................... 8

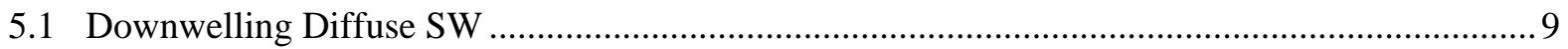

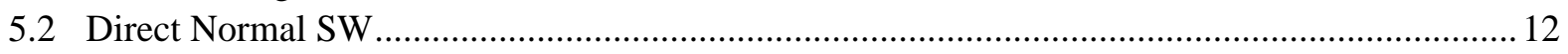

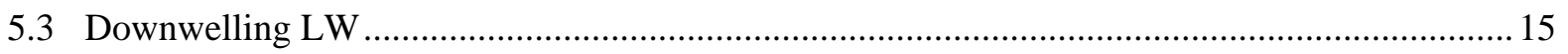

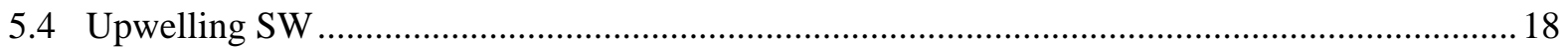

5.5 Upwelling LW

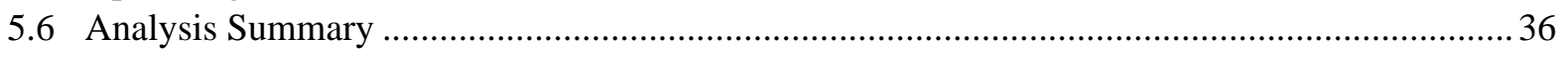

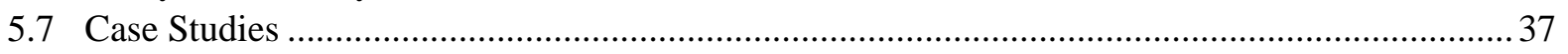

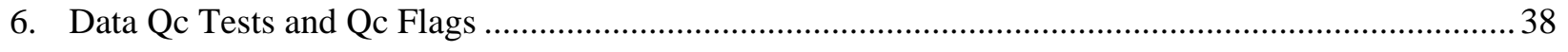

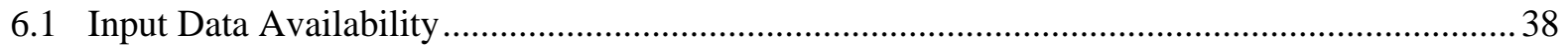

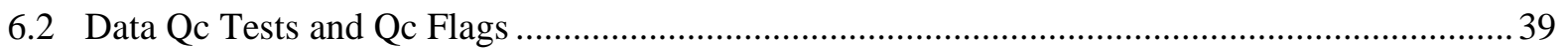

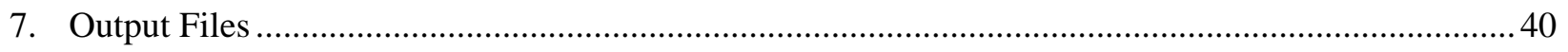

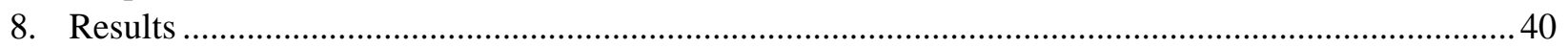

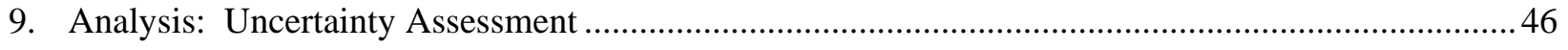

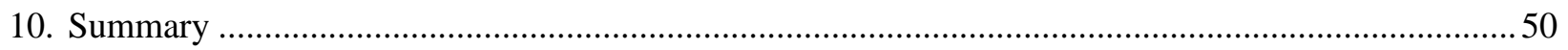

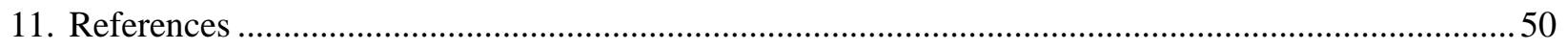




\section{Figures}

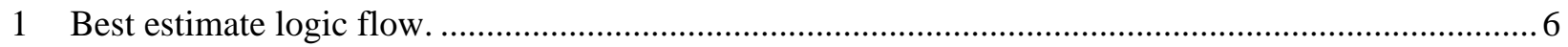

2 Probability distribution of diffuse SW percent differences for instrument pairs................................ 10

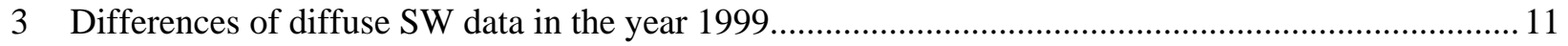

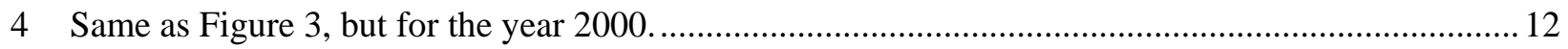

5 Probability distribution of direct normal SW percent differences for instrument pairs. ..................... 13

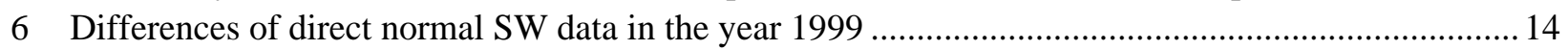

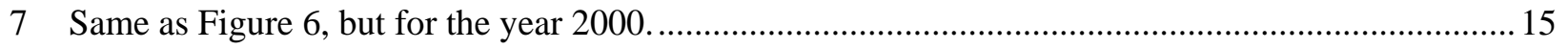

8 Probability distribution of downwelling LW percent differences for instrument pairs........................ 16

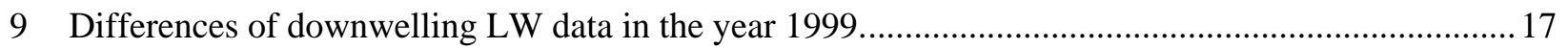

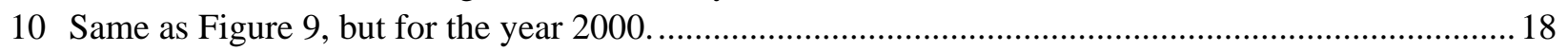

11 Probability distribution of upwelling SW percent differences for instrument pairs............................ 19

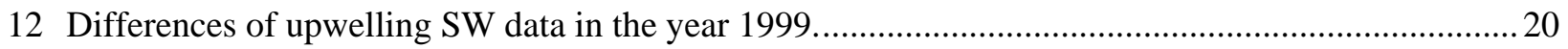

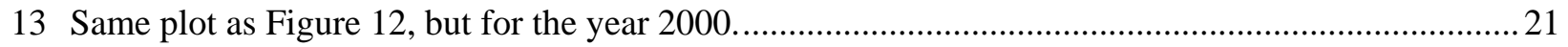

14 SIRS C1 vs. SIRS E13 Upwelling SW data for the year 1999........................................................ 22

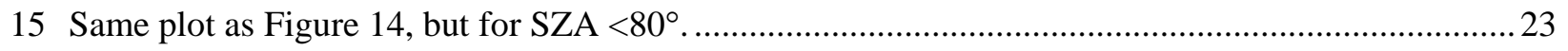

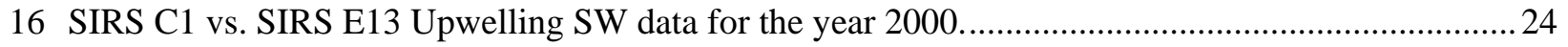

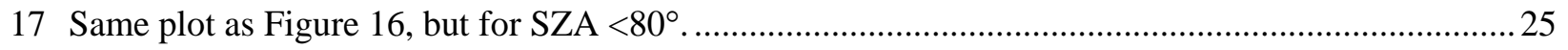

18 Probability distribution of upwelling LW percent differences for instrument pairs............................26

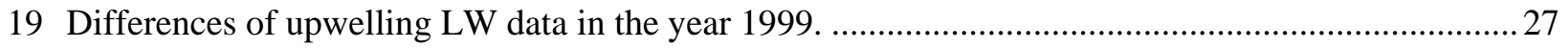

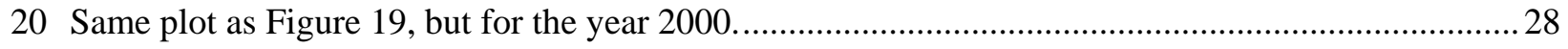

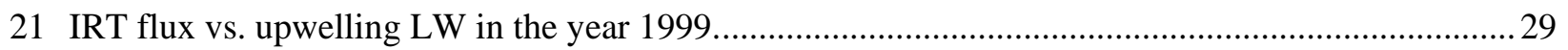

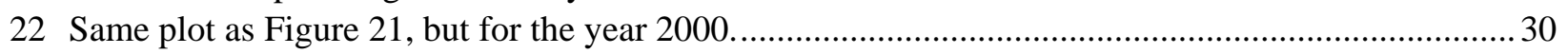

23 IRT flux vs. upwelling LW trend plot for the year 1999.............................................................. 31

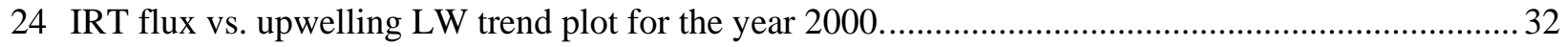

25 15-minute running standard deviation of the ratio of IRT flux over upwelling LW

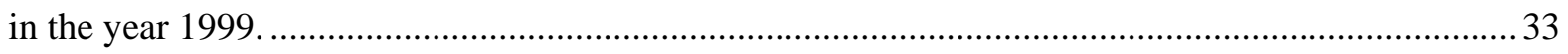

26 Frequency distribution of the standard deviation of the IRT flux vs. upwelling LW data

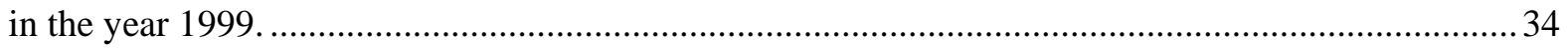

27 15-minute running standard deviation of the ratio of IRT flux over upwelling LW

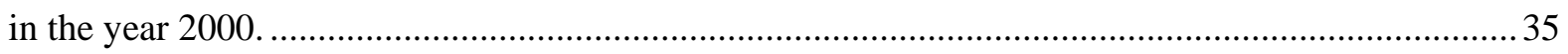

28 Frequency distribution of the standard deviation of the IRT flux vs. upwelling LW data

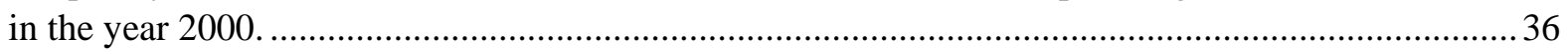

29 Best estimate of downwelling diffuse SW and the differences between best estimate

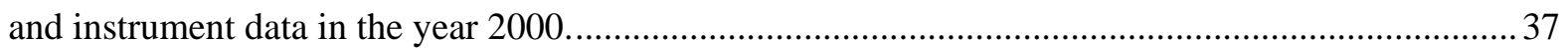

30 Best estimate of direct normal SW and the differences between best estimate

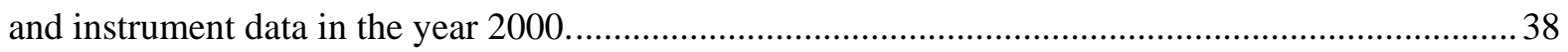

31 Percent of input data available by year and platform. .................................................................. 39

32 Best estimate of diffuse SW and differences between best estimate and instrument data

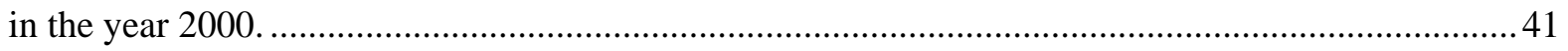

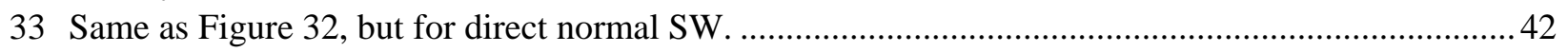

34 Short Direct Normal data measured at three instruments from day 207 to 213................................. 43

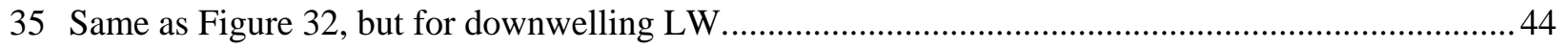

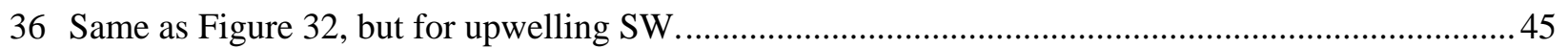




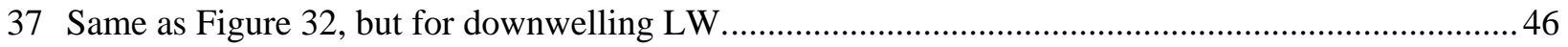

38 Cumulative frequency of instrument differences for diffuse SW in the year 2000 ......................... 47

39 Cumulative frequency of instrument differences for direct normal SW in the year 2000...................48

40 Cumulative frequency of instrument differences for downwelling LW in the year 2000.................. 49

Tables

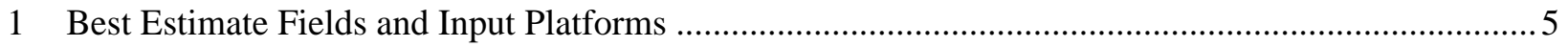

2 Best Estimate Limits and Criteria Used for Data QC Testing ........................................................ 7

3 From Ohmura et al. Showing Calibration Accuracy ...................................................................... 49

4 Summary of the 95\% Level Agreement from 1997 to 2001 ............................................................50 


\section{Introduction}

This document describes some specifics of the algorithm for best estimate evaluation of radiation fluxes at Southern Great Plains (SGP) Central Facility (CF). It uses the data available from the three co-located surface radiometer platforms at the SGP CF to automatically determine the best estimate of the irradiance measurements available. The Best Estimate Flux (BEFlux) value-added procedure (VAP) was previously named Best Estimate ShortWave (BESW) VAP, which included all of the broadband and spectral shortwave (SW) measurements for the SGP CF. In BESW, multiple measurements of the same quantities were handled simply by designating one as the primary measurement and using all others to merely fill in any gaps. Thus, this "BESW" is better termed "most continuous," since no additional quality assessment was applied. We modified the algorithm in BESW to use the average of the closest two measurements as the best estimate when possible, if these measurements pass all quality assessment criteria. Furthermore, we included longwave ( $\mathrm{LW}$ ) fields in the best estimate evaluation to include all major components of the surface radiative energy budget, and renamed the VAP to Best Estimate Flux (BEFLUX1LONG).

\section{Input Data}

The input data for this VAP are from standard Atmospheric Radiation Measurement (ARM) netCDF files. This VAP processes data starting from March 22, 1997, when data from the three CF radiometer systems, SIRS E13, C1, and "BSRN", are all available. In 2001, the diffuse SW instruments were switched to shaded B/W instruments, and the name "baseline surface radiation network (BSRN)" was switched to broadband radiometer station (BRS). Prior to that time, this VAP uses corrected diffuse SW from DiffCorr1Dutt VAP (sgpsirs1duttE13.c1, sgpsirs1duttC1.c1, sgpbsrn1duttC1.c1, and sgpsiros1duttE13.c1) as input. All the input files contain 24-hour data starting from midnight. The time interval for Solar Infrared Station (SIRS) and BSRN/BRS data are 1-minute, while multi-filter radiometer/infrared thermometer (MFR/IRT) data are 20 second.

The input files involved in this VAP are as follows:

1. sgpsirs1duttE13.c1 - used data from August 29, 1997 to February 22, 2001

2. sgpsirs1duttC1.c1 - used data from March 22, 1997 to April 13, 1999

3. sgpbsrn1duttC1.c1 - used data from March 22, 1997 to January 22, 2001

4. sgpsiros1duttE13.c1 - used data from March 22, 1997 to August 28, 1997

5. sgpsirsE13.a1 - always used (if data exists)

6. sgpsirsC1.a1 - always used (if data exists)

7. sgpbsrnC1.a1 - always used (if data exists)

8. sgpsirosE13.a1 - always used (if data exists)

9. sgpsirsE13.a2 - used only if sgpsirsE13.a1 data does not exist

10. sgpsirsC1.a2 - used only if sgpsirsE13.a1 data does not exist

11. sgpbrsC1.a2 - used only if sgpsirsE13.a1 data does not exist

12. sgpirt10mC1.a1 - always used (if data exists) 
13. sgpmfrirt10mC1.b1 - used if sgpirt10mC1.b1 doest not exist

14. sgpmfr10mC1.a1 - used if neither (12) nor (13) datastreams exists

This VAP uses BW library, which standardizes input and output between automated procedures and the netCDF files. It is a standard library used by developers in the ARM Experiment Center and External Data Center. The desired fields from each input file are listed in the beflux1long_retriever.info file described in the next section. All the above listed files can be ordered online from the ARM Archive Center (http://www.archive.arm.gov).

\section{Configuration Files}

The following three configuration files are used for this VAP:

1. beflux1long_main.info

2. beflux1long_retriever.info

3. beflux1long_maker.info

\section{1 beflux1long_main.info}

For a VAP, the value included in _main.info file is always "measurement."

\section{2 beflux1long_retriever.info}

The beflux1long_retriever.info file, specifies what input platforms and fields are included in this VAP. It informs BW to retrieve data from the datastore. We pull in data 7 minutes before and after midnight in order to do the 15-minute running standard deviation calculation later in the code (see Section 4).

The fields pulled in from the first four input files listed in Section 2 (The DiffCorr1Dutt output files) are:

\begin{tabular}{|l|l||}
\hline \multicolumn{1}{|c||}{ Field Name } & \multicolumn{1}{c||}{ Description } \\
\hline dsdh_full_corrected & Fully corrected diffuse SW \\
\hline dsdh_dutton_corrected & Dutton corrected diffuse SW \\
\hline down_short_diffuse_hemisp & Original diffuse SW \\
\hline
\end{tabular}

We use the fully corrected diffuse SW field if it exists (see Younkin and Long 2002, for information on why we choose the full corrected diffuse first, if available); otherwise, use the Dutton corrected diffuse SW. If neither exists, we will use the original diffuse SW data.

The input fields from SIRS/BRS files as listed in Section 2 (5)-(11) are:

\begin{tabular}{|l|l||}
\hline \multicolumn{1}{|c||}{ Field Name } & \multicolumn{1}{c|}{ Description } \\
\hline short_direct_normal & Shortwave Direct Normal Irradiance \\
\hline down_short_diffuse_hemisp & Downwelling Shortwave Diffuse Hemispheric Irradiance \\
\hline
\end{tabular}




\begin{tabular}{|l|l||}
\hline \multicolumn{1}{|c||}{ Field Name } & \multicolumn{1}{c||}{ Description } \\
\hline up_short_hemisp & Upwelling Shortwave Hemispheric Irradiance \\
\hline up_long_hemisp & Upwelling Longwave Hemispheric Irradiance \\
\hline down_long_hemisp & Downwelling Longwave Hemispheric Irradiance \\
\hline down_long_hemisp_shaded & Downwelling Longwave Hemispheric Irradiance \\
\hline \hline
\end{tabular}

The last two fields are the same but named differently in old (before April 1, 2001) and new netCDF files.

The field required from sgpmfr10mC1.a1 is:

\begin{tabular}{|c|l||}
\hline \multicolumn{1}{|c||}{ Field Name } & \multicolumn{1}{c|}{ Description } \\
\hline sfc_temp & Effective Surface (Skin) Temperature \\
\hline
\end{tabular}

The field required from sgpmfrirt10mC1.b1 and sgpirt10mC1.a1 is:

\begin{tabular}{|l|l|}
\hline \multicolumn{1}{|c|}{ Field Name } & \multicolumn{1}{c|}{ Description } \\
\hline sfc_ir_temp & Effective Surface (Skin) Temperature \\
\hline
\end{tabular}

\section{3 beflux1long_maker.info}

The beflux1long_maker.info file specifies the output netCDF file names and the fields included in the output files. It includes all the formatting information for the output netCDF files.

The best estimate Flux VAP produces two primary sets of 1-minute resolution output files: The first one (beflux1long) contains the best estimate of downwelling SW direct and diffuse, downwelling LW, and upwelling SW and LW irradiances, the net surface radiation, and the broadband SW surface albedo, along with the corresponding QC flag for each field, and the differences between the two instrument data used for averaging. The other file (qcflux1long) contains the above irradiance fields and the differences between the best estimate and each instrument data.

The output fields and their units and description in the first output netCDF file (beflux1long) are:

\begin{tabular}{|l|l|l||}
\hline \multicolumn{1}{|c|}{ Field Name } & \multicolumn{1}{c||}{ Units } & \multicolumn{1}{c||}{ Description } \\
\hline base_time & $\begin{array}{l}\text { Seconds } \\
\text { since }\end{array}$ & 1970-1-1 0:00:00 0:00 \\
\hline time_offset & Seconds & Time offset from base_time \\
\hline down_short_hemisp & $\mathrm{Wm}^{-2}$ & Downwelling Shortwave hemispheric Irradiance \\
\hline down_long_hemisp & $\mathrm{Wm}^{-2}$ & Downwelling Longwave Hemispheric Irradiance \\
\hline short_direct_normal & $\mathrm{Wm}^{-2}$ & Shortwave Direct Normal Irradiance \\
\hline down_short_diffuse_hemisp & $\mathrm{Wm}^{-2}$ & Downwelling Shortwave Diffuse Hemispheric Irradiance \\
\hline up_short_hemisp & $\mathrm{Wm}^{-2}$ & Upwelling Shortwave Hemispheric Irradiance \\
\hline up_long_hemisp & $\mathrm{Wm}^{-2}$ & Upwelling Longwave Hemispheric Irradiance \\
\hline net_surface_radiation & $\mathrm{Wm}^{-2}$ & Net Surface Radiation \\
\hline \hline
\end{tabular}




\begin{tabular}{|l|l|l||}
\hline \multicolumn{1}{|c|}{ Field Name } & \multicolumn{1}{c||}{ Units } & \multicolumn{1}{c||}{ Description } \\
\hline albedo & unitless & Broadband Shortwave Surface Albedo \\
\hline zenith & degrees & Solar Zenith Angle \\
\hline down_long_hemisp_flag & unitless & QC Flag for down_long_hemisp \\
\hline short_direct_normal_flag & unitless & QC Flag for short_direct_normal \\
\hline down_short_diffuse_hemisp_flag & unitless & QC Flag for down_short_diffuse_hemisp \\
\hline up_short_hemisp_flag & unitless & QC Flag for up_short_hemisp \\
\hline up_long_hemisp_flag & unitless & QC Flag for up_long_hemisp \\
\hline short_direct_normal_diff & Wm ${ }^{-2}$ & $\begin{array}{l}\text { Difference between the two instrument data used for } \\
\text { averaging }\end{array}$ \\
\hline down_short_diffuse_hemisp_diff & Wm ${ }^{-2}$ & $\begin{array}{l}\text { Difference between the two instrument data used for } \\
\text { averaging }\end{array}$ \\
\hline down_long_hemisp_diff & Wm ${ }^{-2}$ & $\begin{array}{l}\text { Difference between the two instrument data used for } \\
\text { averaging }\end{array}$ \\
\hline up_short_hemisp_diff & Wm ${ }^{-2}$ & $\begin{array}{l}\text { Difference between the two instrument data used for } \\
\text { averaging }\end{array}$ \\
\hline up_long_hemisp_diff & Wm ${ }^{-2}$ & $\begin{array}{l}\text { Difference between the two instrument data used for } \\
\text { averaging }\end{array}$ \\
\hline lat & degrees & North latitude \\
\hline lon & degrees & East longitude \\
\hline alt & meters $^{\text {Altitude }}$ \\
\hline
\end{tabular}

The output fields in the second output netCDF file (qcflux1long) are:

\begin{tabular}{|l|l|l||}
\hline \multicolumn{1}{|c|}{ Field Name } & \multicolumn{1}{|c||}{ Units } & \multicolumn{1}{c||}{ Description } \\
\hline base_time & $\begin{array}{l}\text { Seconds } \\
\text { since }\end{array}$ & $1970-1-1$ 0:00:00 0:00 \\
\hline time_offset & Seconds & Time offset from base_time \\
\hline down_short_hemisp & $\mathrm{Wm}^{-2}$ & Downwelling Shortwave hemispheric Irradiance \\
\hline down_long_hemisp & $\mathrm{Wm}^{-2}$ & Downwelling Longwave Hemispheric Irradiance \\
\hline short_direct_normal & $\mathrm{Wm}^{-2}$ & Shortwave Direct Normal Irradiance \\
\hline down_short_diffuse_hemisp & $\mathrm{Wm}^{-2}$ & Downwelling Shortwave Diffuse Hemispheric Irradiance \\
\hline up_short_hemisp & $\mathrm{Wm}^{-2}$ & Upwelling Shortwave Hemispheric Irradiance \\
\hline up_long_hemisp & $\mathrm{Wm}^{-2}$ & Upwelling Longwave Hemispheric Irradiance \\
\hline net_surface_radiation & $\mathrm{Wm}^{-2}$ & Net Surface Radiation \\
\hline albedo & $\mathrm{unitless}^{-2}$ & Broadband Shortwave Surface Albedo \\
\hline zenith & $\mathrm{degrees}^{-2}$ & Solar Zenith Angle \\
\hline qcdirsirse & $\mathrm{Wm}^{-2}$ & $\begin{array}{l}\text { difference between best estimate and SIRS E13 } \\
\text { short_direct_normal }\end{array}$ \\
\hline qcdirsirsc & $\begin{array}{l}\text { difference between best estimate and SIRS C1 } \\
\text { short_direct_normal }\end{array}$ \\
\hline qcdirbrs & $\mathrm{Wm}^{-2}$ & $\begin{array}{l}\text { difference between best estimate and BRS } \\
\text { short_direct_normal }\end{array}$ \\
\hline \hline
\end{tabular}




\begin{tabular}{|l|l|l||}
\hline \multicolumn{1}{|c|}{ Field Name } & \multicolumn{1}{|c||}{ Units } & \multicolumn{1}{c||}{ Description } \\
\hline qcdifsirse & $\mathrm{Wm}^{-2}$ & $\begin{array}{l}\text { difference between best estimate and SIRS E13 } \\
\text { down_short_diffuse_hemisp }\end{array}$ \\
\hline qcdifsirsc & $\mathrm{Wm}^{-2}$ & $\begin{array}{l}\text { difference between best estimate and SIRS C1 } \\
\text { down_short_diffuse_hemisp }\end{array}$ \\
\hline qcdifbrs & $\mathrm{Wm}^{-2}$ & $\begin{array}{l}\text { difference between best estimate and BRS } \\
\text { down_short_diffuse_hemisp }\end{array}$ \\
\hline qcdlfsirse & $\mathrm{Wm}^{-2}$ & $\begin{array}{l}\text { difference between best estimate and SIRS E13 } \\
\text { down_long_hemisp }\end{array}$ \\
\hline qcdlfsirsc & $\mathrm{Wm}^{-2}$ & $\begin{array}{l}\text { difference between best estimate and SIRS C1 } \\
\text { down_long_hemisp }\end{array}$ \\
\hline qcdlfbrs & $\mathrm{Wm}^{-2}$ & $\begin{array}{l}\text { difference between best estimate and BRS } \\
\text { down_long_hemisp }\end{array}$ \\
\hline qcuhfsirse & $\mathrm{Wm}^{-2}$ & $\begin{array}{l}\text { difference between best estimate and SIRS E13 } \\
\text { up_short_hemisp }\end{array}$ \\
\hline qcuhfsirsc & $\mathrm{Wm}^{-2}$ & $\begin{array}{l}\text { difference between best estimate and SIRS C1 } \\
\text { up_shorthemisp }\end{array}$ \\
\hline qculfsirse & $\mathrm{Wm}^{-2}$ & $\begin{array}{l}\text { difference between best estimate and SIRS E13 } \\
\text { up_long_hemisp }\end{array}$ \\
\hline qculfsirsc & $\mathrm{Wm}^{-2}$ & $\begin{array}{l}\text { difference between best estimate and SIRS C1 } \\
\text { up_long_hemisp }\end{array}$ \\
\hline lat & $\mathrm{degrees}^{\text {North latitude }}$ \\
\hline lon & $\mathrm{degrees}^{\text {alt }}$ & $\mathrm{meters}^{\text {East longitude }}$ \\
\hline
\end{tabular}

\section{Algorithm}

This VAP uses the data available from the three co-located surface radiometer platforms at the SGP CF to automatically determine the best irradiance measurements available. It processes data starting from March 22, 1997, when data from all three radiometer systems, SIRS E13, C1, and "BSRN," are available. In 2001, the diffuse SW instruments were switched to shaded B/W instruments, and the name "BSRN" was switched to BRS. Prior to that time, this VAP uses corrected diffuse SW from the DiffCorr1Dutt VAP (sgpsirs1duttE13.c1, sgpsirs1duttC1.c1, and sgpbsrn1duttC1.c1) as input.

Table 1 lists the fields being calculated in this VAP and the input platforms involved. The data from the input platforms are first pegged down to 1-minute samples. Then they are compared to decide which will be used in the best estimate calculation.

Table 1. Best Estimate Fields and Input Platforms

\begin{tabular}{|l|l||}
\hline \multicolumn{1}{|c||}{ Field Description } & \multicolumn{1}{c|}{ Input Platforms } \\
\hline Downwelling Shortwave Diffuse Hemispheric Irradiance & SIRS E13, C1, \& BSRN/BRS \\
\hline Shortwave Direct Normal Irradiance & SIRS E13, C1, \& BSRN/BRS \\
\hline Downwelling Shortwave Hemispheric Irradiance & Sum of Direct \& Diffuse \\
\hline Downwelling Longwave Hemispheric Irradiance & SIRS E13 \& C1, BSRN/BRS \\
\hline
\end{tabular}




\begin{tabular}{|l|l||}
\hline Upwelling Shortwave Hemispheric Irradiance & SIRS E13 \& C1, MFR10M \\
\hline Upwelling Longwave Hemispheric Irradiance & SIRS E13 \& C1, IRT \\
\hline Net Surface Radiation & $\begin{array}{l}\text { (Downwelling SW - Upwelling SW) } \\
\text { + (Downwelling LW - Upwelling LW) }\end{array}$ \\
\hline Broadband Shortwave Surface Albedo & Upwelling/Downwelling SW \\
\hline
\end{tabular}

Figure 1 shows the logic flow for this VAP. The upper chart is for diffuse and direct normal SW, and downwelling LW, where three measurement data from SIRS E13, C1, and BRS are available. The lower chart describes the logic flow for upwelling LW and SW, where only two measurements from SIRS E13 and $\mathrm{C} 1$ are available.

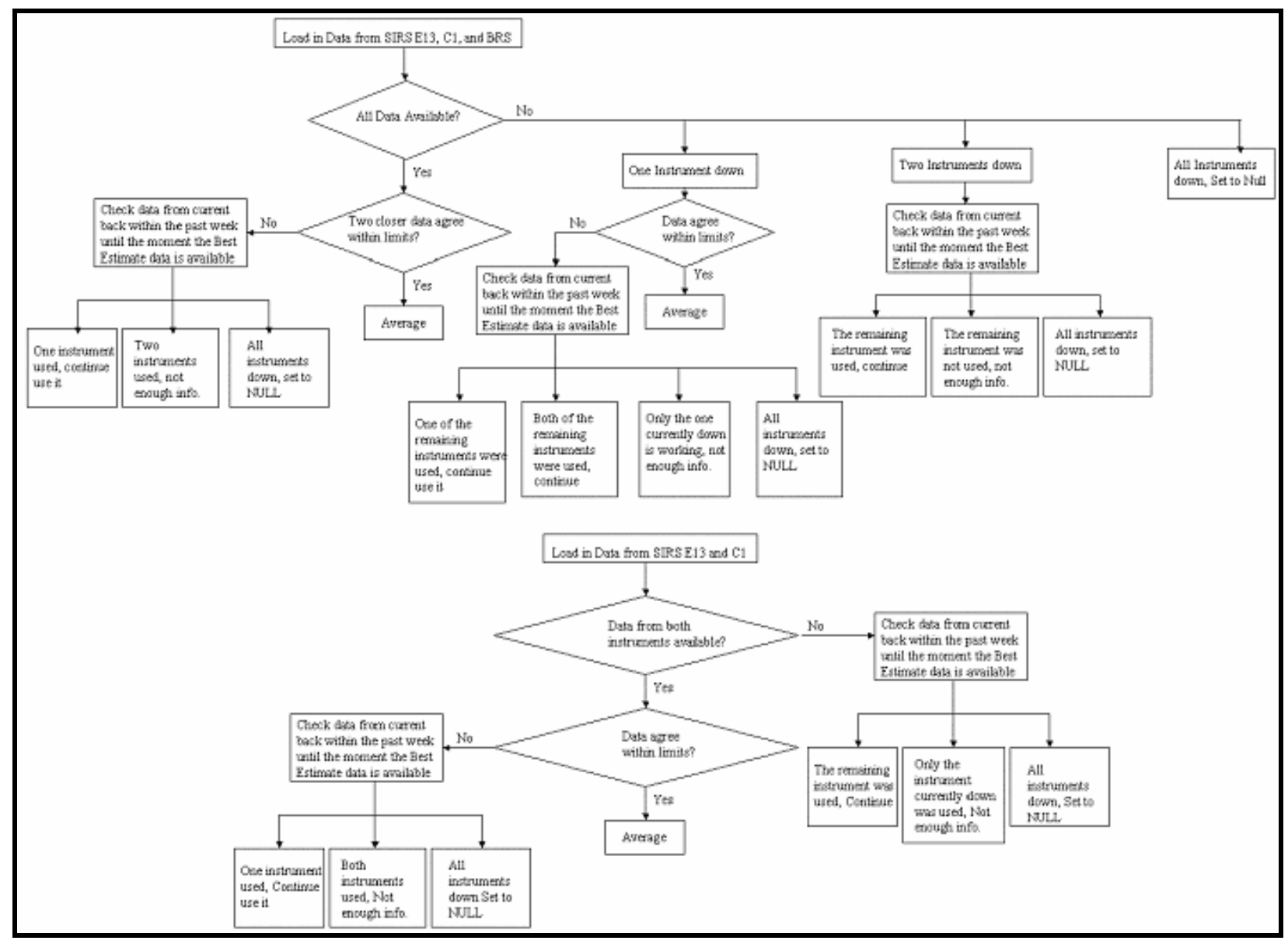

Figure 1. Best estimate logic flow. Upper chart: Diffuse and direct normal SW, and downwelling LW. Lower chart: Upwelling LW and SW. The limits for each field are listed in Table 2. 
Table 2. Best Estimate Limits and Criteria Used for Data QC Testing

\begin{tabular}{|c|c|c|c|c|c|c|c|c|}
\hline \multirow[t]{2}{*}{ Field Name } & \multicolumn{2}{|c|}{$\begin{array}{c}\text { Prct of Data } \\
\text { meet the } \\
\text { Criteria }\end{array}$} & \multirow[t]{2}{*}{ Criteria } & \multicolumn{2}{|c|}{ Prct of Data meet the Criteria } & \multirow[t]{2}{*}{ Criteria } & \multicolumn{2}{|c|}{$\begin{array}{l}\text { Prct of Data } \\
\text { meet either } \\
\text { Criteria }\end{array}$} \\
\hline & 1999 & 2000 & & 1999 & 2000 & & 1999 & 2000 \\
\hline Diffiuse SW & $54.8 \%$ & $54.3 \%$ & Data Diff. $<10 \%$ & $93.6 \%$ & $86.1 \%$ & Dat a Diff. $<5 \mathrm{~W} / \mathrm{m}^{2}$ & $99.6 \%$ & $99.9 \%$ \\
\hline Direct Normal SW & $46.0 \%$ & $43.2 \%$ & Data Diff. $<5 \%$ & $96.5 \%$ & $86.4 \%$ & Dat a Diff. $<5 \mathrm{~W} / \mathrm{m}^{2}$ & $99.5 \%$ & $99.5 \%$ \\
\hline Dowrwilling LW & $99.6 \%$ & $99.2 \%$ & Data Diff $<2 \%$ & $98.8 \%$ & $94.9 \%$ & Dat a Diff. $<5 \mathrm{~W} / \mathrm{m}^{2}$ & $99.7 \%$ & $99.2 \%$ \\
\hline Upwelling SW & $98.8 \%$ & $97.9 \%$ & $\begin{array}{l}\mathrm{ABS}(\mathrm{C} 1 / \mathrm{E} 13-1)<0.2 \text { for } \\
\text { zenith }<80\end{array}$ & $99.5 \%$ & $99.7 \%$ & $\begin{array}{l}\text { Dat a Diff. }<10 \% \text { or } 5 \mathrm{~W} / \mathrm{m} 2 \text { for } \\
\text { zenith } 280\end{array}$ & $98.8 \%$ & $97.9 \%$ \\
\hline Upwelling LW & $99.7 \%$ & $90.5 \%$ & Data Diff. $<4 \%$ & C1: $98.0 \%$ E13: $99.0 \%$ & $\begin{array}{ll}\text { C1: } 90.2 \% & \text { E13: } 99.9 \%\end{array}$ & STD DEV $<0.01$ & $99.7 \%$ & $90.5 \%$ \\
\hline
\end{tabular}

A brief description of the logic used in the best estimate calculation follows:

For fields measured by three instruments (i.e., diffuse and direct normal SW, and downwelling LW, upper part of the flow chart).

1. If data from all three instruments are available, then check to see if the two closest data meet the criteria (for criteria/limits used for each field, see Table 2). If the two closest data meet the criteria, average the two; else check the data from the current back within the past week until the moment the best estimate data is available

a. if only one instrument data was used as the best estimate at that moment, continue use it

b. if two instrument data were averaged as the best estimate at that moment, then we do not have enough information to decide the best estimate value

c. if the best estimate value is unavailable even until the last iteration of checking, then set the current estimate data to NULL.

2. If only one instrument is down, check to see if the two remaining data meet the criteria. If yes, average the two; else, check the data from the current back within the past week until the moment the best estimate data is available. As an example, say that the BRS data is missing. Then if the data from SIRSC \& SIRSE agree within the criteria, average the two. If they do not agree to within the allowable criteria, look back and
a. if SIRSE and SIRSC data were averaged, continue
b. if BRS and SIRSC data were averaged, use SIRSC
c. if BRS and SIRSE data were averaged, use SIRSE
d. if only SIRSE or SIRSC was used, continue
e. if only BRS was used, not enough information to decide
f. if none was working, set to NULL.

3. If only one instrument is working, e.g., only BRS is available. Check the data from the current back within the past week until the moment the best estimate data is available

a. if at least one instrument was working and BRS was used in the calculation, then continue use it 

b. if at least one instrument was working and BRS was not used in the calculation, then we do not have enough information to decide
c. if all instruments were down, set to NULL.

4. If all instruments are down, set to NULL.

For fields measured by two instruments (upwelling SW and LW, lower part of the flow chart).

1. If data from both instruments are available, then check to see if the two datasets meet the criteria (see Section 5), if yes, average, else, check the data from the current back within the past week until the moment the best estimate data is available
a. if only one instrument was working, continue use it
b. if both instruments were working or best estimate value is undecided due to lack of information, then current best estimate value cannot be decided since there is not enough information
c. if averaged data is missing until the end of the iteration, then set the current best estimate value to NULL.

2. If only one instrument is working, e.g., SIRSE is working, check the data from the current back within the past week until the moment the best estimate data is available
a. if both instruments were working or only SIRSE was working, continue use SIRSE
b. if only SIRSC was working, we do not have enough information to decide the best estimate value
c. if both instruments were down until the end of the iteration, then set the current best estimate value to NULL.

\section{Criteria/Limits Used in Best Estimate Calculation}

It is an axiom in data quality that if one has only one measurement of a quantity, then quality assessment beyond testing against known physical limits is problematic (case 1). If one has two measurements of the same or very similar quantities, then one can both test against physical limits, and compare the two measurements (case 2). If both measurements are physically possible, but disagree beyond the expected accuracy of the measuring instruments, then it is difficult to identify which is the "good" measurement. If one has three or more measurements of the same or very similar quantities, and one measurement disagrees with the rest beyond the expected accuracy of the measuring instruments, then probability arguments can be used to infer that the "odd" measurement is most likely the "bad" one (case 3). In the BE Flux VAP, the best estimate of diffuse and direct normal SW, and downwelling LW fall under case 3, where the parameters were measured by three CF radiometer systems - SIRS E13, C1, and "BRS". Upwelling SW and LW data only involve two measurements from SIRS E13 and C1, thus fall under case 2. In the case of upwelling LW, the two measurements are compared with the downward facing IRT measurement to achieve more control over data quality.

The criteria/limits used in the best estimate calculation were obtained by statistical analysis with 2 years of data in 1999 and 2000. Frequency distributions of agreement between the two that agreed the best over 
these 2 years were examined. In general, it was easy to pick out a percent or $\mathrm{Wm}^{-2}$ level where the agreement covered the "good" data, as shown in Table 2. For diffuse SW, we set the criteria as the data differences $<10 \%$ or $5 \mathrm{Wm}^{-2}$. 99.6\% data in 1999 and 99.9\% data in 2000 fall within this criteria. For direct normal SW, 99.5\% data in both 1999 and 2000 fall within the criteria of data differences $<5 \%$ or $5 \mathrm{Wm}^{-2}$. In the case of downwelling, LW, 99.7\% data in 1999 and $99.2 \%$ data in 2000 meet the criteria of data difference $<2 \%$ or $5 \mathrm{Wm}^{-2}$. For upwelling SW, only two instruments were used for these measurements and we do not have a third measurement as a reference. After comparing the two measurements from SIRS E13 and SIRS C1, we decided the ratio of these two measurements is a good indicator of data agreement during daytime (solar zenith angle $[\mathrm{SZA}]<80^{\circ}$ ). For upwelling $\mathrm{LW}$, the two measurements from SIRS E13 and C1 were compared with the downward facing IRT measurement. The results show that the standard deviation of the ratio of brightness temperature calculated from the upwelling pyrgeometer over the corresponding downward facing IRT measurement is a good indicator of data agreement. The following Sections (5.1 to 5.5) discussed the detailed analysis of the 1999 and 2000 data and the criteria/limits listed in Table 2.

\subsection{Downwelling Diffuse SW}

Figure 2 shows the probability distributions of the percent differences between the diffuse SW data of the instrument pairs used for the best estimate calculation, for 1999 and 2000. The upper plot is for year 1999 and the lower plot is for year 2000. We see that the percent of data differences less than $10 \%$ are $98 \%$ for both years. Note that this plot only included the daytime measurements (SZA less than $80^{\circ}$ ), since the daytime data is what we really concerned about.

Figures 3 and 4 are the differences between the two instruments used for averaging, in 1999 and 2000, respectively. Similar to Figure 2, only daytime measurements (SZA less than $80^{\circ}$ ) are analyzed here. For data with best estimate greater than $20 \mathrm{Wm}^{-2}$ (colored red in the upper plots of Figures 3 and 4), the differences are mostly less than $10 \%$. For data with best estimate less than or equal to $20 \mathrm{Wm}^{-2}$ (colored blue in the lower plots of Figures 3 and 4), the differences of the two instrument data used for averaging are generally well below $5 \mathrm{Wm}^{-2}$.

In conclusion, the best estimate calculation criterion is set to $10 \%$ or $5 \mathrm{Wm}^{-2}$ i.e., we take the average of the two instrument data as the best estimate only if the differences between the two instruments are less than $10 \%$ or $5 \mathrm{Wm}^{-2}$, whichever is greater. Otherwise, we will check back through the past week's data and decide what to do. 
Downwelling Shortwave Diffuse Hemispheric Irradiance
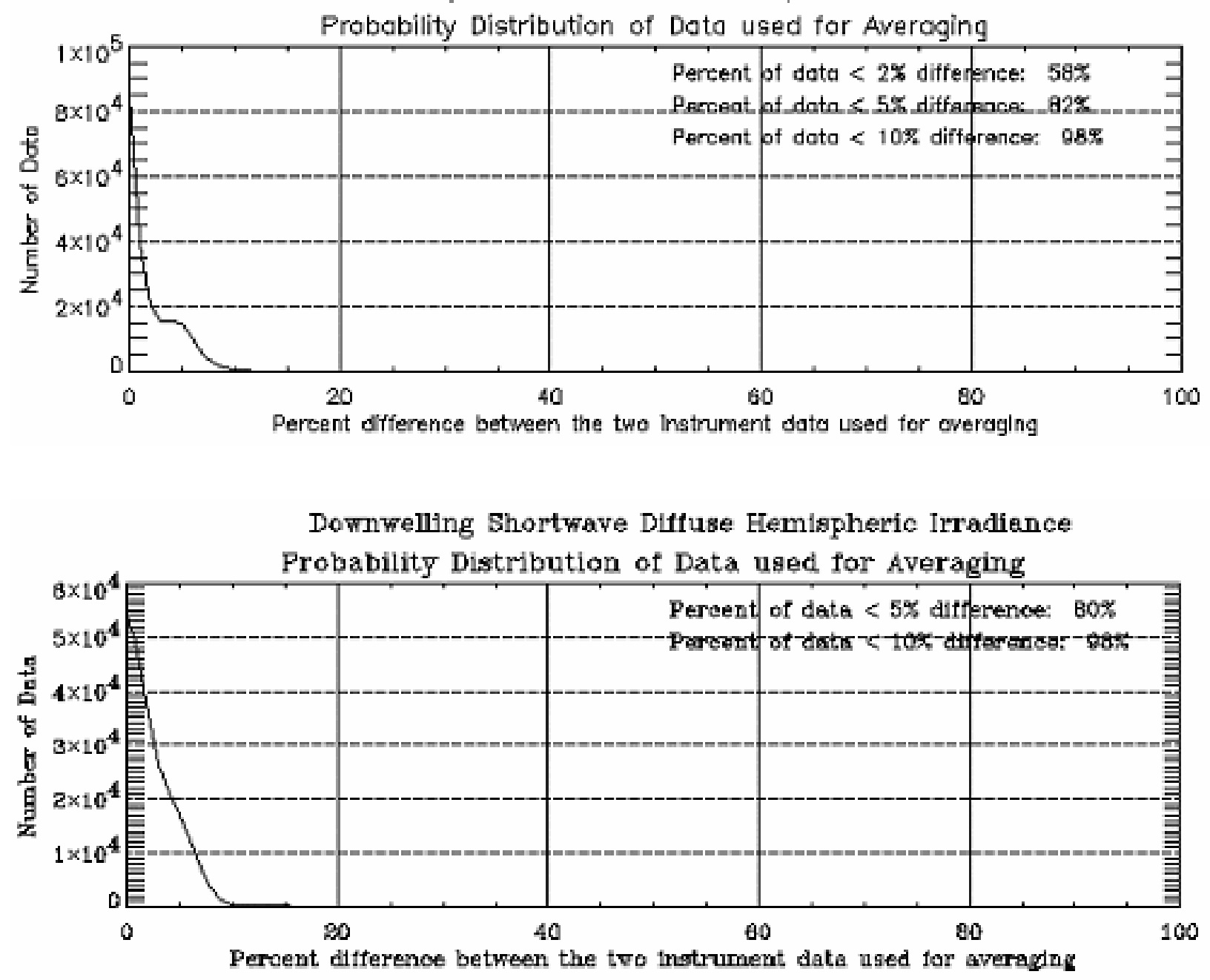

Figure 2. Probability distribution of diffuse SW percent differences for instrument pairs. Upper plot: year 1999; Lower plot: year 2000. 
Downwelling Shortwaye Diftuge Hemlspherie Irradiance
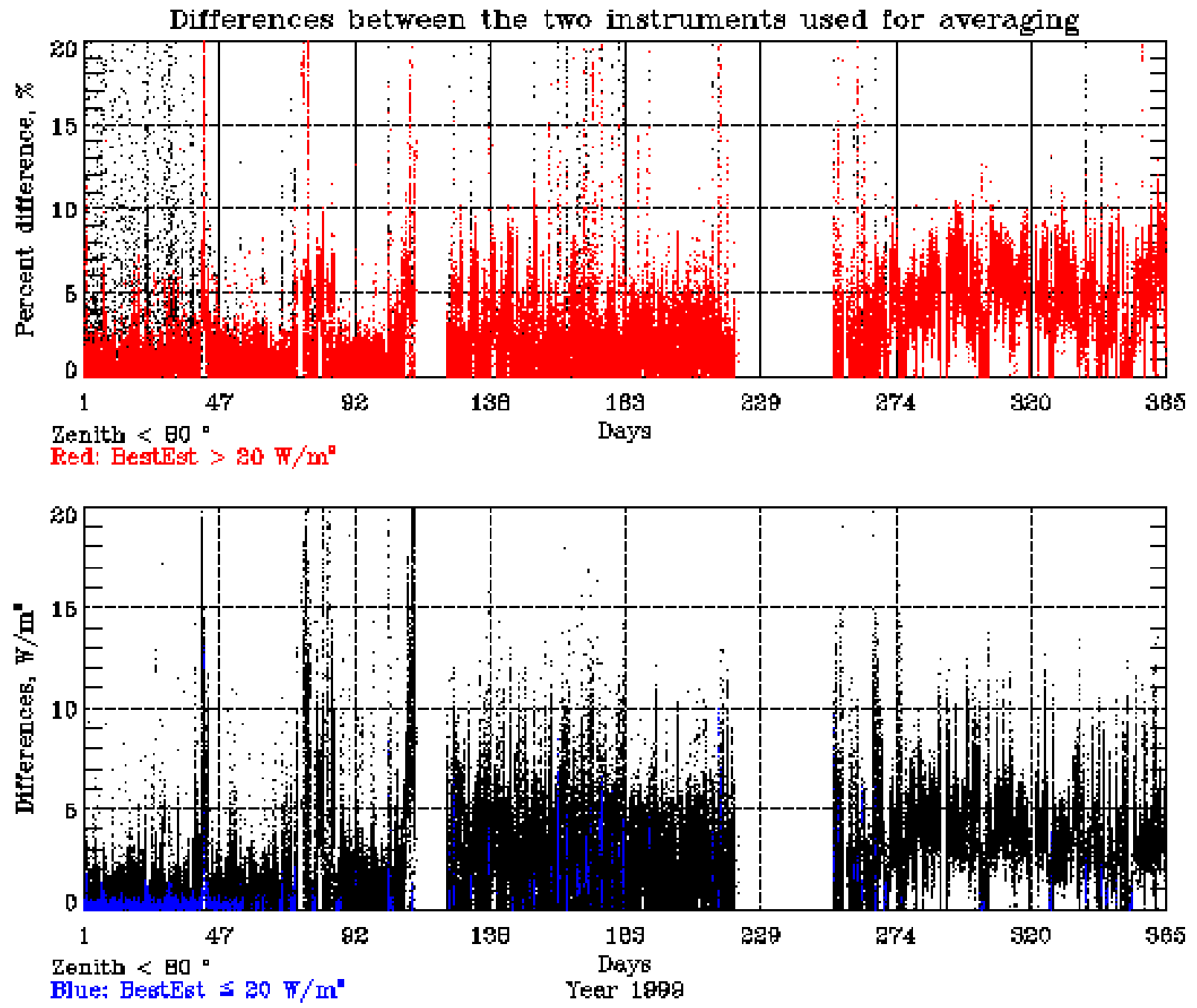

Figure 3. Differences of diffuse SW data in the year 1999. Upper plot: percent differences of the data with the SZA less than $80^{\circ}$, the data with the best estimate value greater than $20 \mathrm{Wm}^{-2}$ are colored red; Lower plot: absolute differences of the data with the SZA less than $80^{\circ}$, the data with the best estimate value less or equal to $20 \mathrm{Wm}^{-2}$ are colored blue. 

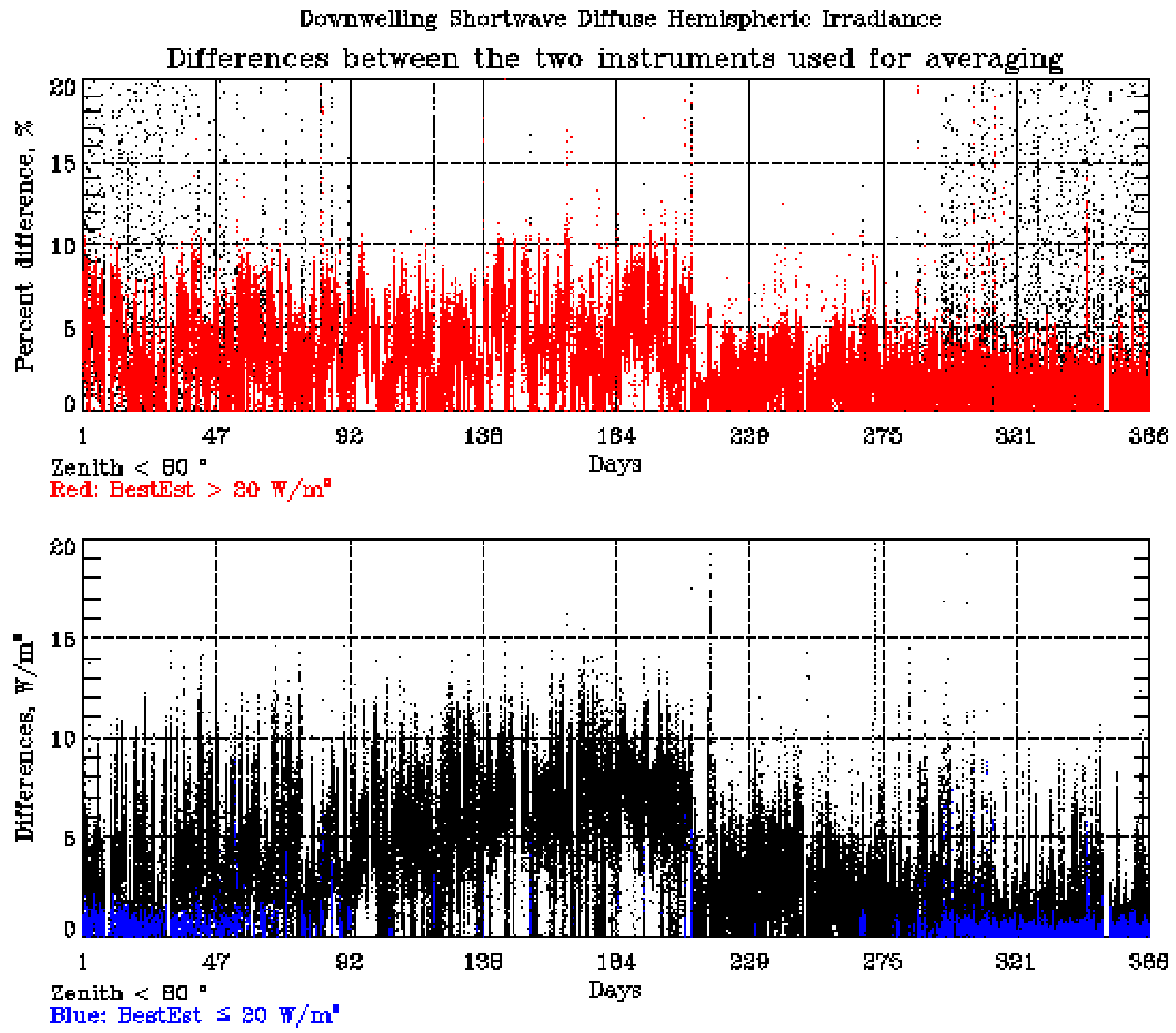

Figure 4. Same as Figure 3, but for the year 2000.

\subsection{Direct Normal SW}

Figure 5 shows the percent differences between the direct normal SW data of the instrument pairs used for the best estimate calculation, for 1999 and 2000. Here the data are also screened for daytime measurements only SZA less than $80^{\circ}$ ), as with the diffuse SW data. The upper plot is for year 1999 and the lower plot is for year 2000. The percent of data differences less than $5 \%$ are $82 \%$ for year 1999 and $79 \%$ for year 2000. Note that these data are unscreened for magnitude, thus, includes periods when the direct normal SW magnitude was small and 5\% of the value is smaller still.

Similar to Figures 3 and 4, Figures 6 and 7 are the differences between the two instruments used for averaging for direct normal SW. Most data differences fall below $5 \%$ when the best estimate values are greater than $20 \mathrm{Wm}^{-2}$ (colored red in the upper plots of Figures 6 and 7). In the year 2000 from day 200 to day 366, the absolute differences between the data are up to $15 \mathrm{Wm}^{-2}$ or more. However, the percent 
differences remain low (less than 5\%). For data with best estimate less than or equal to $20 \mathrm{Wm}^{-2}$ (colored blue in the lower plots of Figures 6 and 7), the differences are well below $3 \mathrm{Wm}^{-2}$.

Thus, the best estimate calculation criteria for direct normal SW is set to $\underline{5 \% \text { or } 5 \mathrm{Wm}^{-2}}$, whichever is greater.
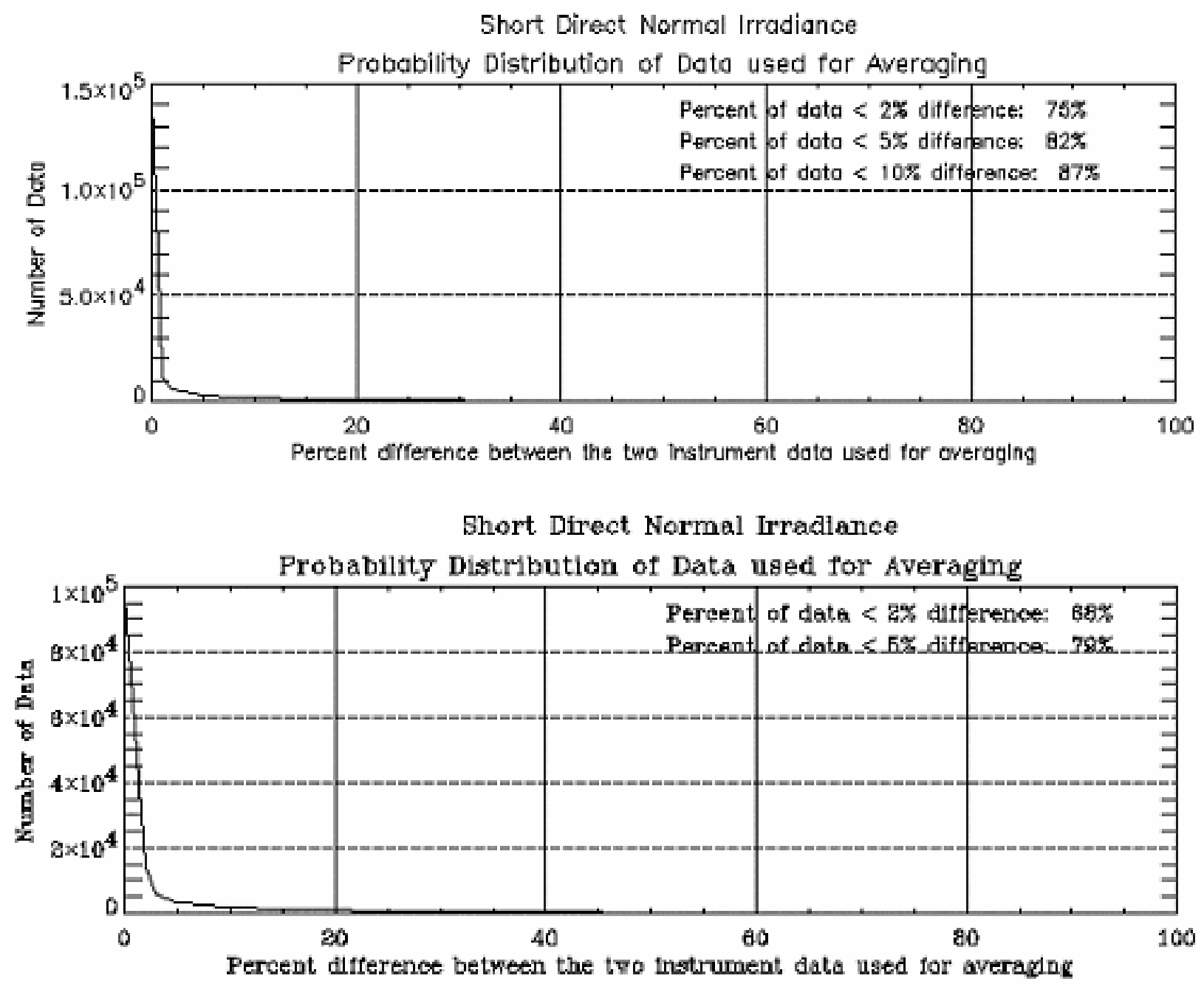

Figure 5. Probability distribution of direct normal SW percent differences for instrument pairs. Upper plot: year 1999; Lower plot: year 2000. 
Short Direat Normal Irradiance

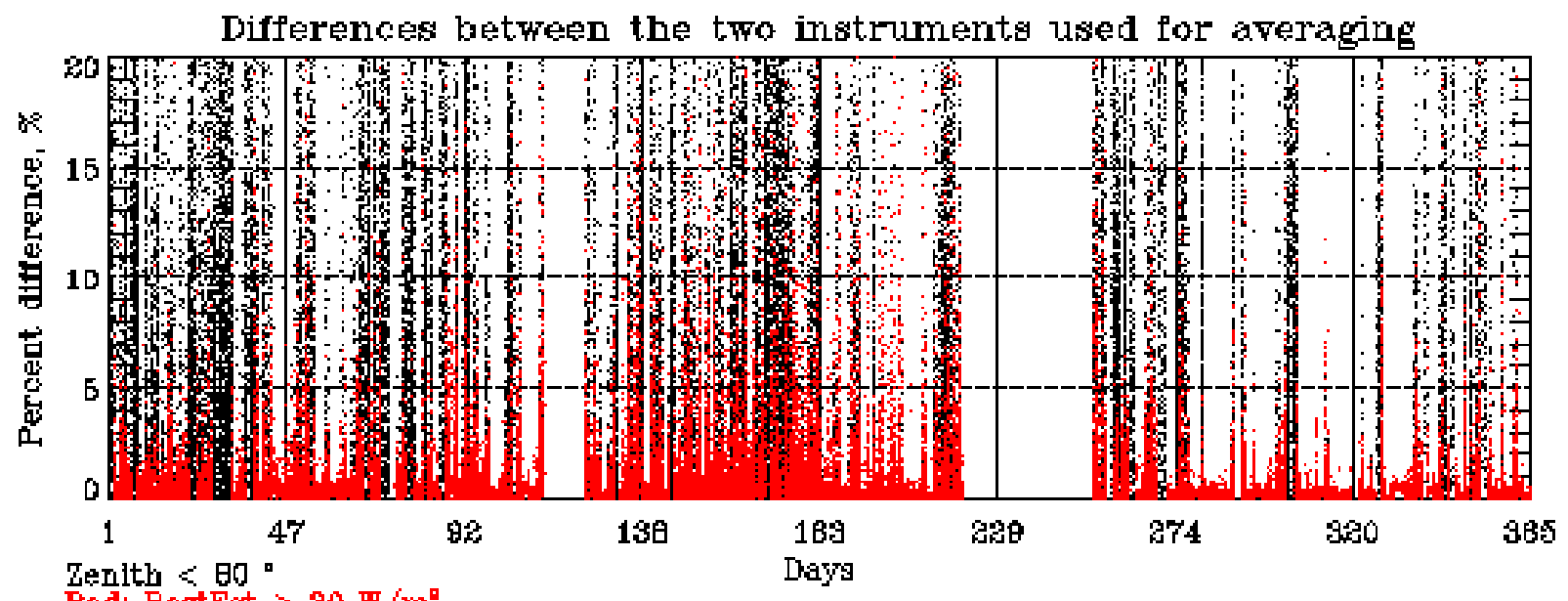

Red: Bestest $>20 \mathrm{~T} / \mathrm{m}^{\mathrm{D}}$

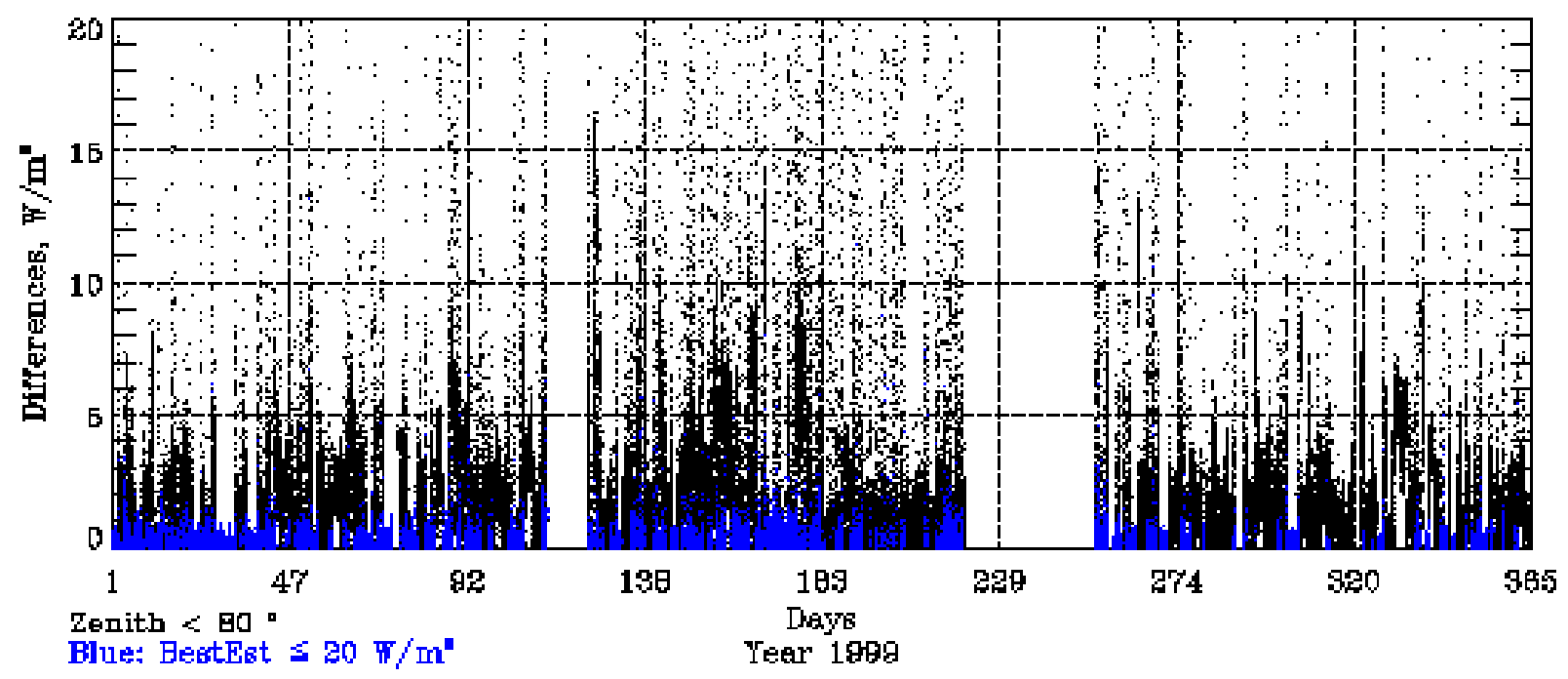

Figure 6. Differences of direct normal SW data in the year 1999. Upper plot: percent differences of the data with the SZA less than $80^{\circ}$, the data with the best estimate value greater than $20 \mathrm{Wm}^{-2}$ are colored red; Lower plot: absolute differences of the data with the SZA less than $80^{\circ}$, the data with the best estimate value less or equal to $20 \mathrm{Wm}^{-2}$ are colored blue. 
Short Direat Normal Irradiance
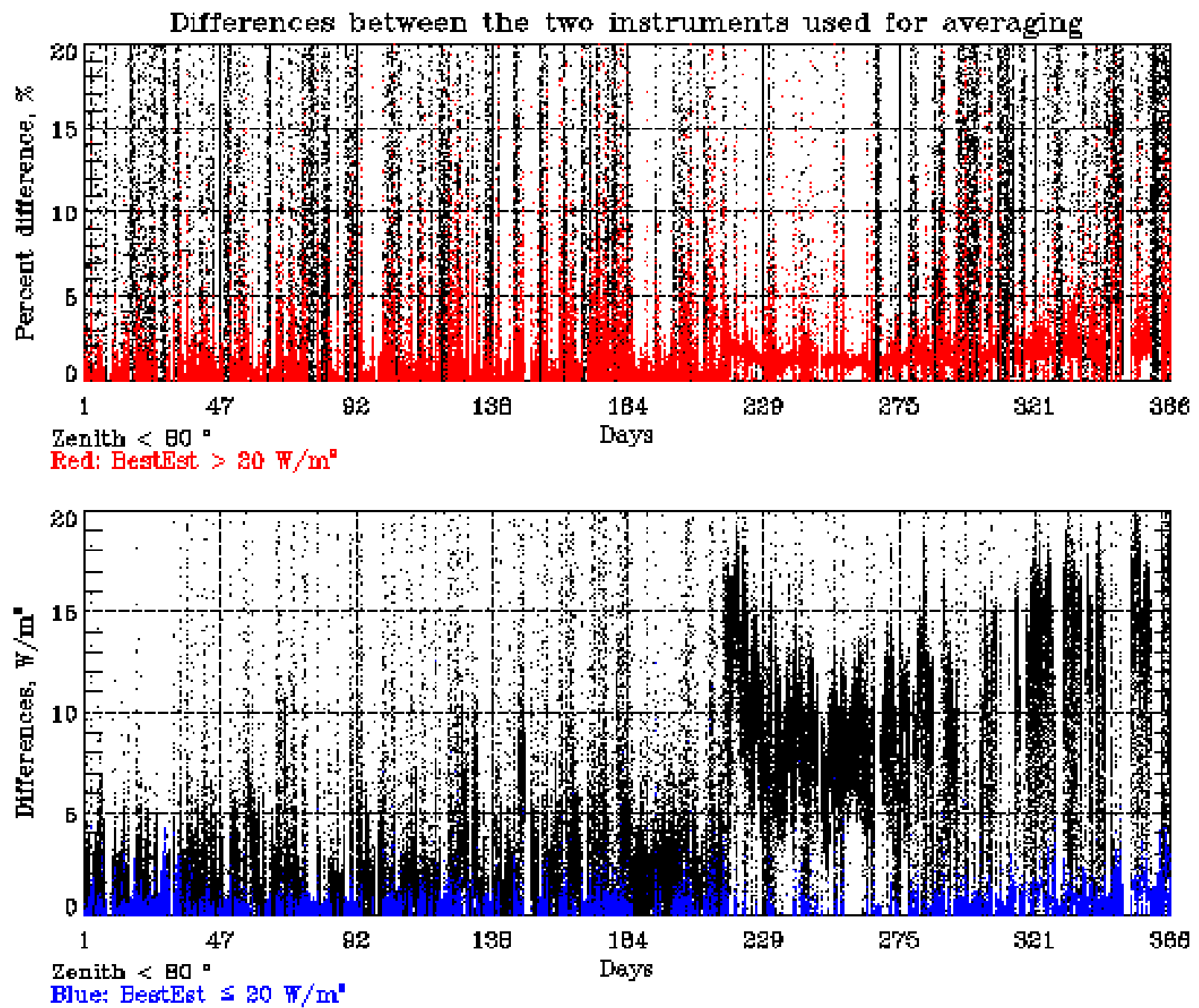

Figure 7. Same as Figure 6, but for the year 2000.

\subsection{Downwelling LW}

Same as Figure 2 to Figure 4, Figure 8 to Figure 10 are plots of the percent differences between the downwelling LW data of the instrument pairs used for the best estimate calculation. The plots show that most of the data fall within a 2\% limit (99\% for 1999 and 98\% for 2000). Figures 9 and 10 indicate most of the data differences are below $2 \%$ or $5 \mathrm{Wm}^{-2}$. In Figure 10, we see that the data differences from day 138 to 184 are apparently greater than other time period. By checking the original SIRS and BRS data we found that from 17:30 GMT on May 9, 2000, to 16:30 GMT on June 30, 2000, SIRS C1 data were well over $500 \mathrm{Wm}^{2}$, while SIRS E13 and BRS data were both around $400 \mathrm{Wm}^{-2}$. Therefore, SIRS C1 data is considered an outlier at this time period and the greater differences shown in Figure 10 is the differences of SIRS E13 and BRS data, compared to the smaller differences between SIRS E13 and C1 before this time period. After day 184, SIRS C1 data returned to normal but still not behaving very steady. This is shown in the data differences immediately following this time period. 
In conclusion, the criteria set for downwelling $\mathrm{LW}$ is $\underline{2 \%}$ or $5 \mathrm{Wm}^{-2}$, whichever is greater.
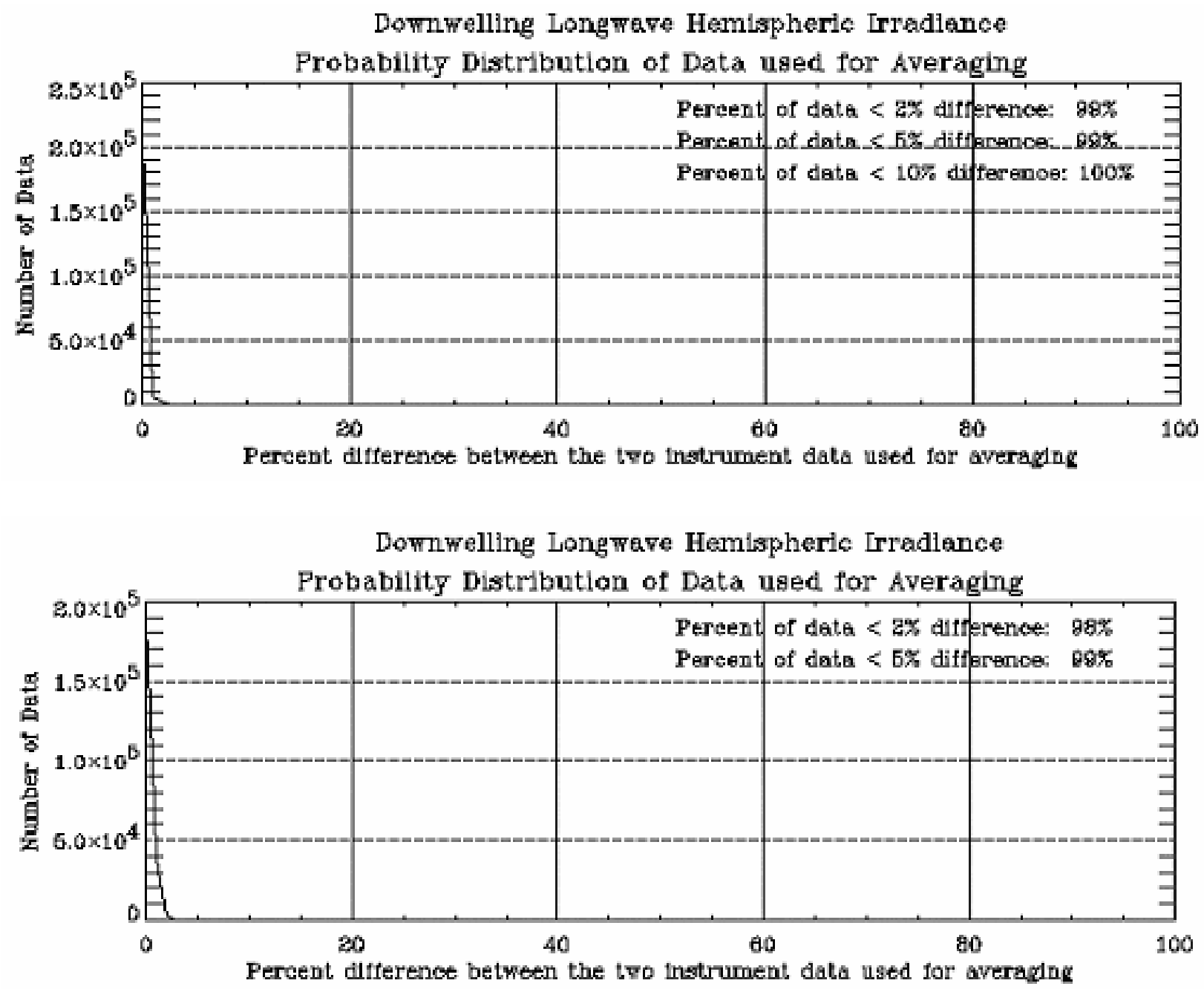

Figure 8. Probability distribution of downwelling LW percent differences for instrument pairs. Upper plot: year 1999; Lower plot: year 2000. 
Dotnwelling Langwave Hemispherie Irradiance

Differences between the two instruments used for averaging
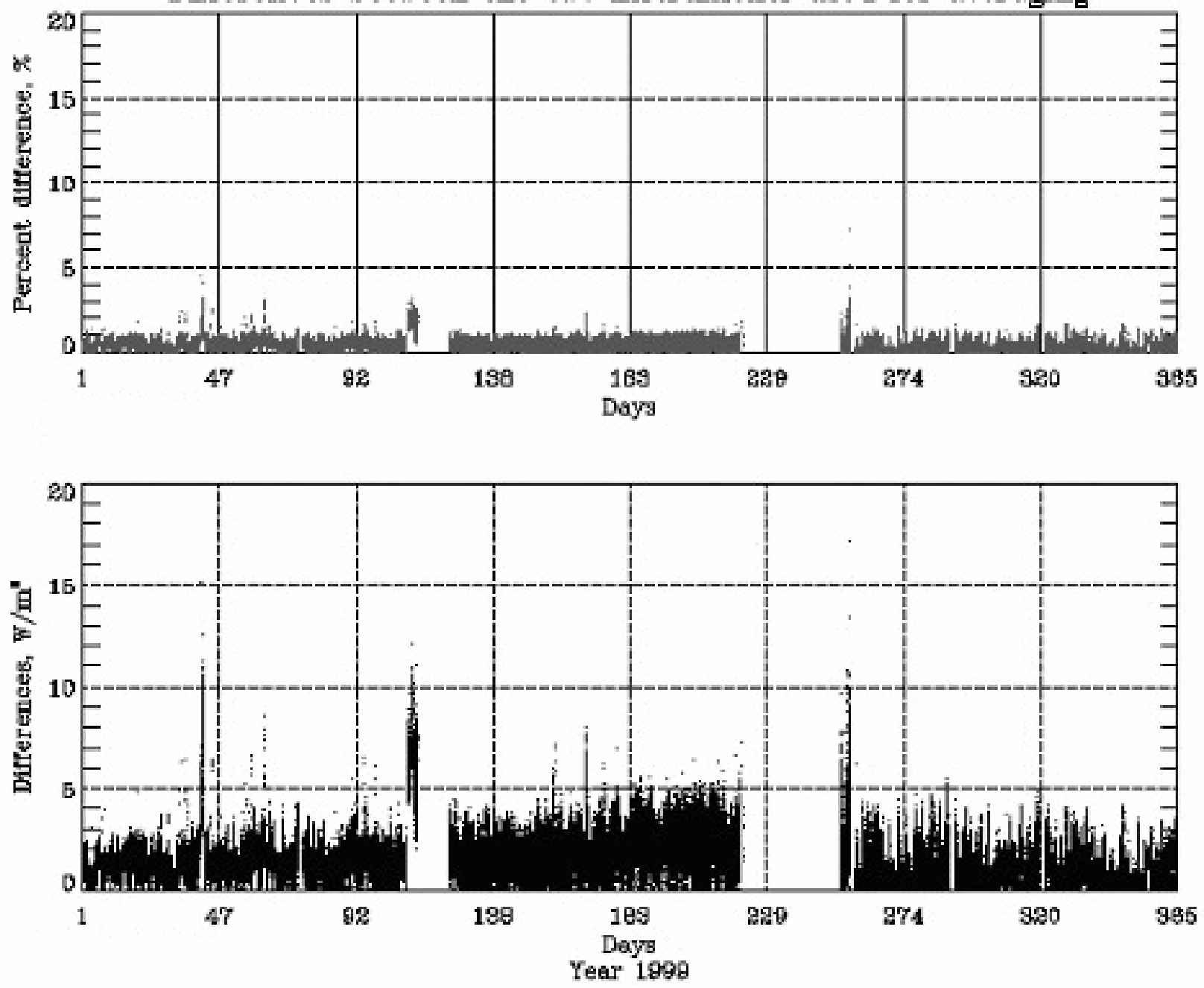

Figure 9. Differences of downwelling LW data in the year 1999. Upper plot: percent differences of the data with the SZA less than $80^{\circ}$, the data with the best estimate value greater than $20 \mathrm{Wm}^{-2}$ are colored red; Lower plot: absolute differences of the data with the SZA less than $80^{\circ}$, the data with the best estimate value less or equal to $20 \mathrm{Wm}^{-2}$ are colored blue. 

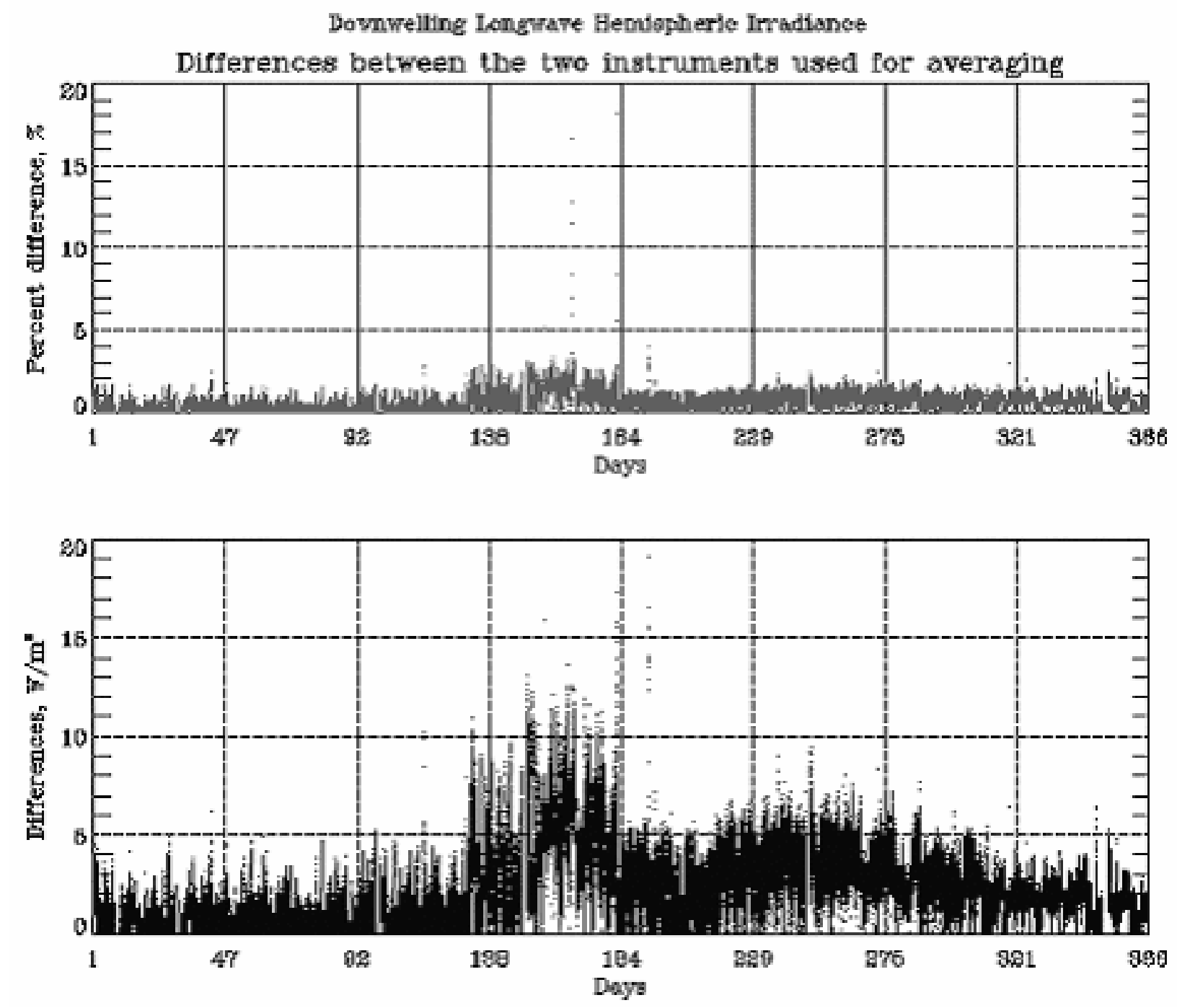

Figure 10. Same as Figure 9, but for the year 2000.

\section{$5.4 \quad$ Upwelling SW}

Figure 11 to Figure 13 are similar plots as Figure 2 to Figure 4, but for upwelling SW. The plots show that most data fall within $20 \%$ or $15 \mathrm{Wm}^{-2}$. After examining the ratio of the two instrument data, i.e., (SIRS C1 data) / (SIRS E13 data), we decided the ratio is a better data quality check. Figures 14 and 15 show the ratio between SIRS C1 and E13 and the probability distribution of the ratio for year 1999. Figure 14 plotted all the data for year 1999 and Figure 15 only plotted data with SZA less than 80 degrees. Figures 16 and 17 are the same plots as Figures 14 and 15 but for year 2000. We can see that the increased distribution appearing at abs $(\mathrm{C} 1 / \mathrm{E} 13-1)=1$ in Figure 14 disappears in Figure 15. This indicates that the increased distribution represents data of SZA greater than 80 degrees. This phenomenon is also true for year 2000 data, as shown in Figures 16 and 17 . Figures 15 and 17 also show that the percentage of the data falling within 0.2 of C1/E13 = 1 is $98 \%$ for year 1999 and $96 \%$ for year 2000. 
Therefore, the C1/E13 ratio is a good criterion to use for determining good data when the SZA is less than $80^{\circ}$. Thus, for data with SZA $<80^{\circ}$, we use abs(C1/E13-1) $<0.2$ as a check for data quality. For data with $\mathrm{SZA}>=80^{\circ}$, we choose the criteria of the data differences less than $10 \%$ or $5 \mathrm{Wm}^{-2}$, whichever is greater.
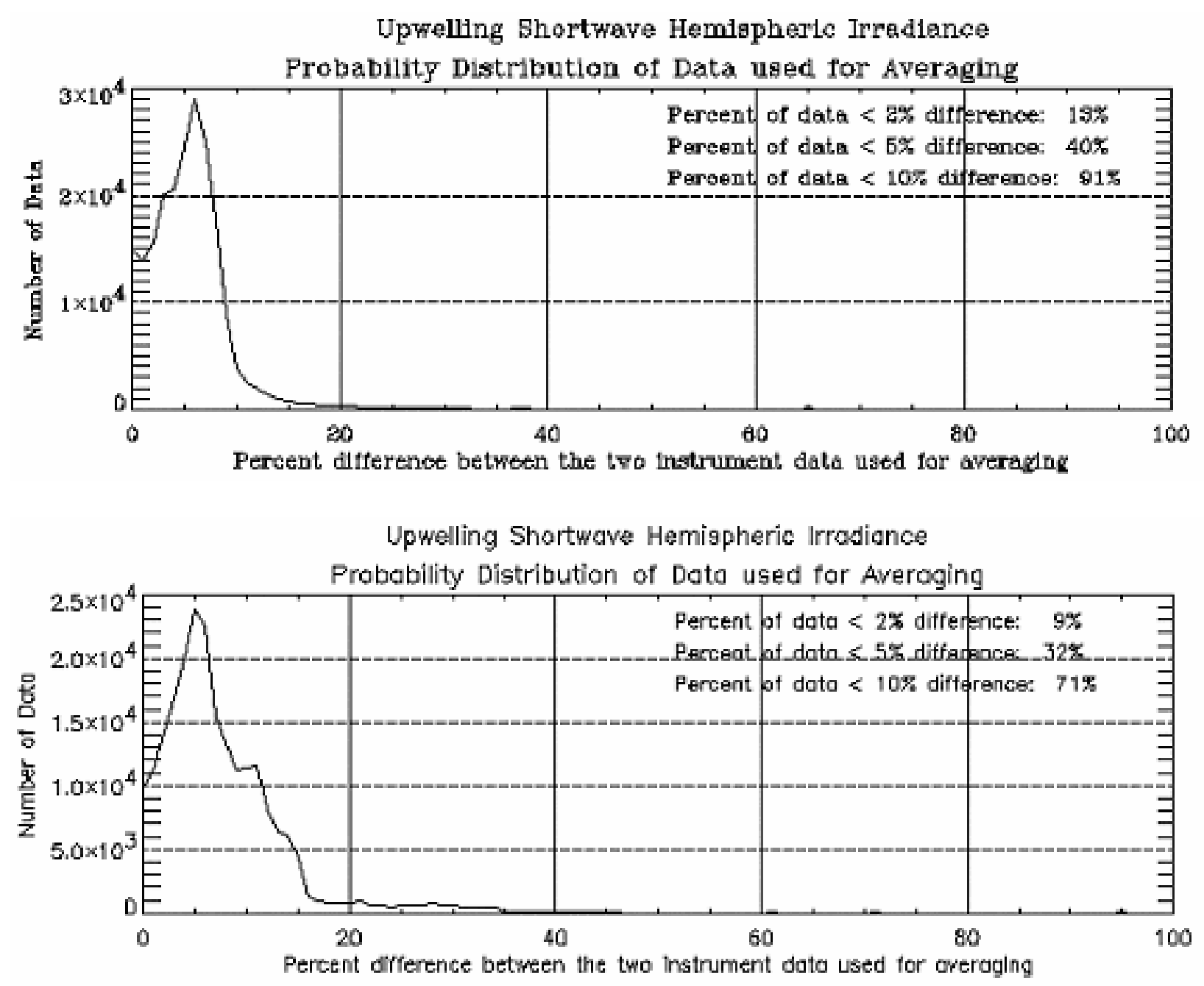

Figure 11. Probability distribution of upwelling SW percent differences for instrument pairs. Upper plot: year 1999; Lower plot: year 2000. 
Upwelling Shortwave Hemispherle Irradiance

Differences between the two instruments used for averaging

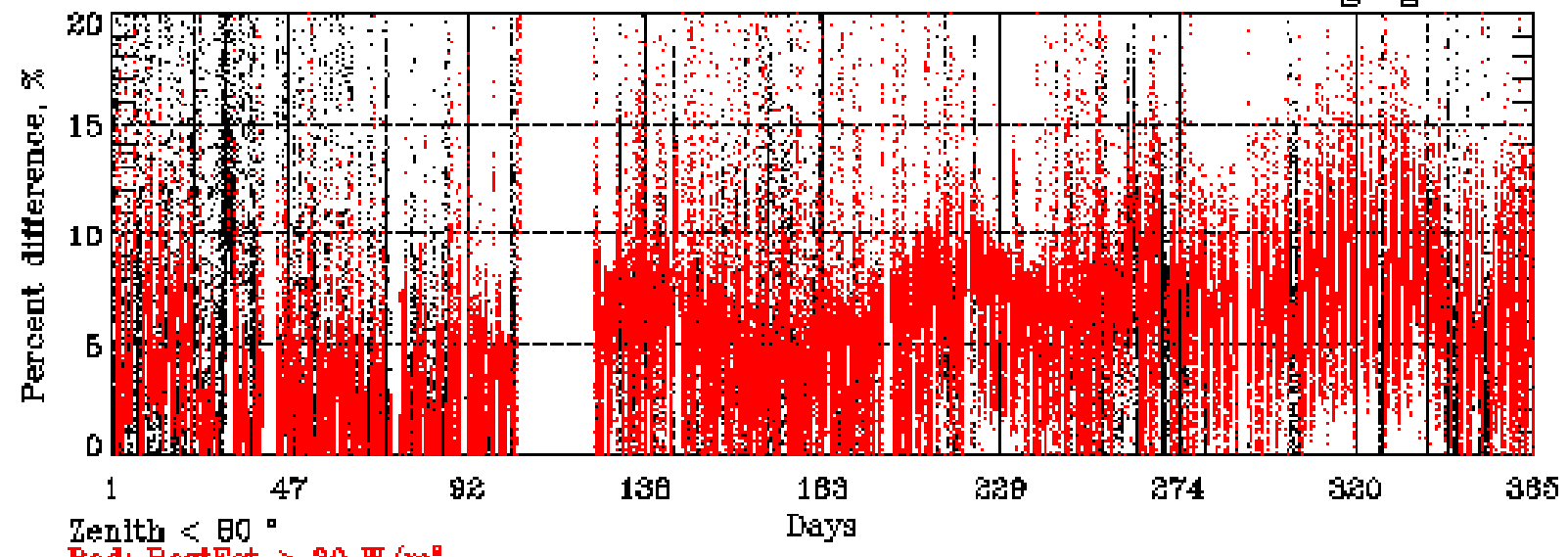

Red: Best.tist $>20$ T $/ \mathrm{m}^{\mathrm{D}}$

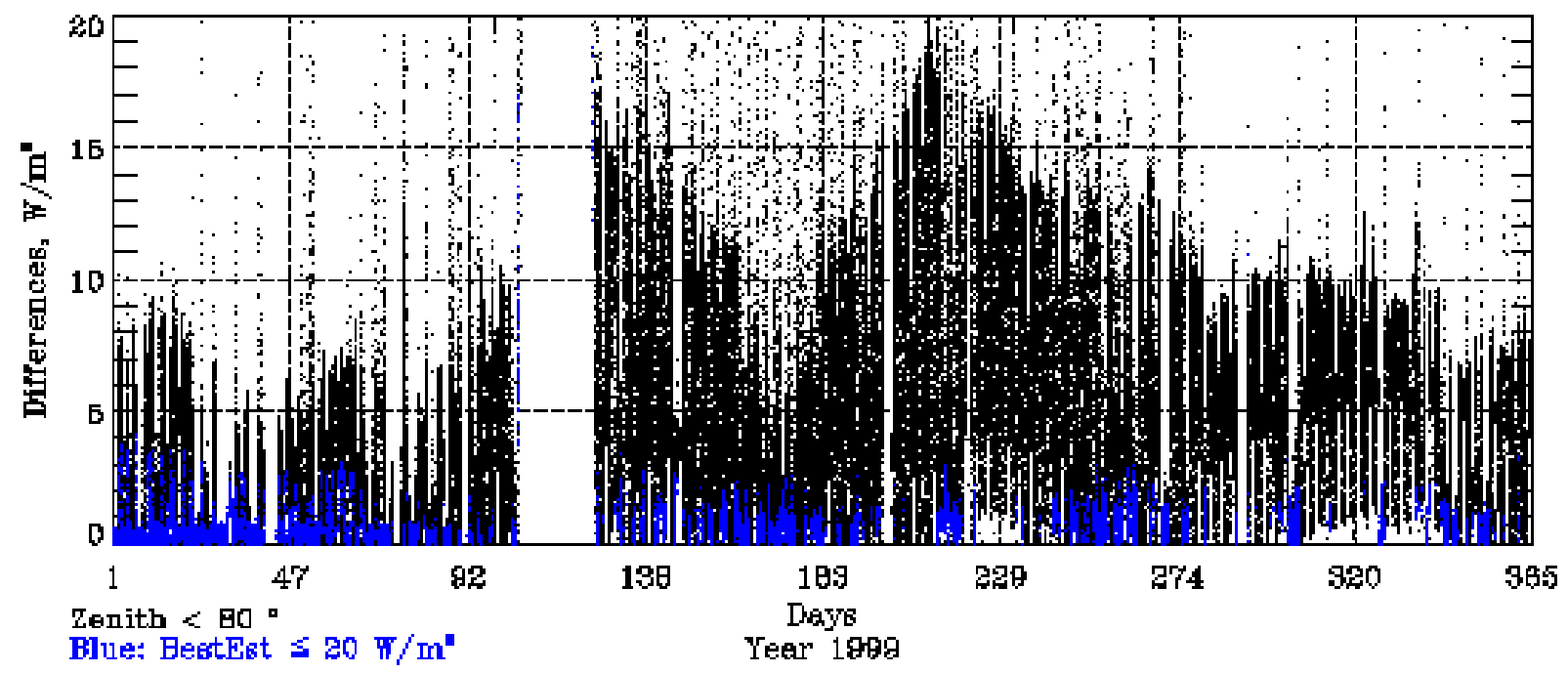

Figure 12. Differences of upwelling SW data in the year 1999. Upper plot: percent differences of the data with the SZA less than $80^{\circ}$, the data with the best estimate value greater than $20 \mathrm{Wm}^{-2}$ are colored red; Lower plot: absolute differences of the data with the SZA less than $80^{\circ}$, the data with the best estimate value less or equal to $20 \mathrm{Wm}^{-2}$ are colored blue. 

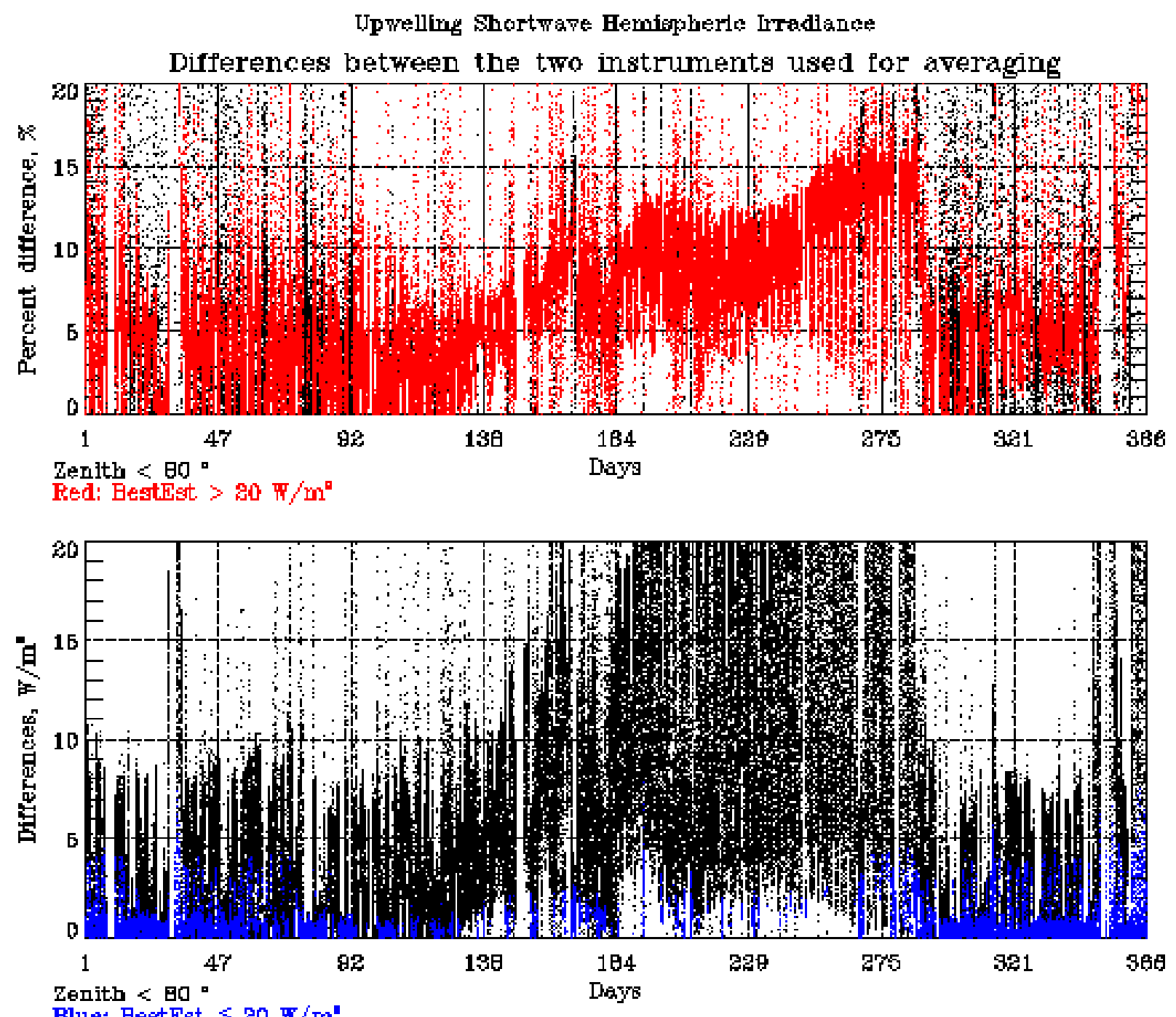

Figure 13. Same plot as Figure 12, but for the year 2000. 

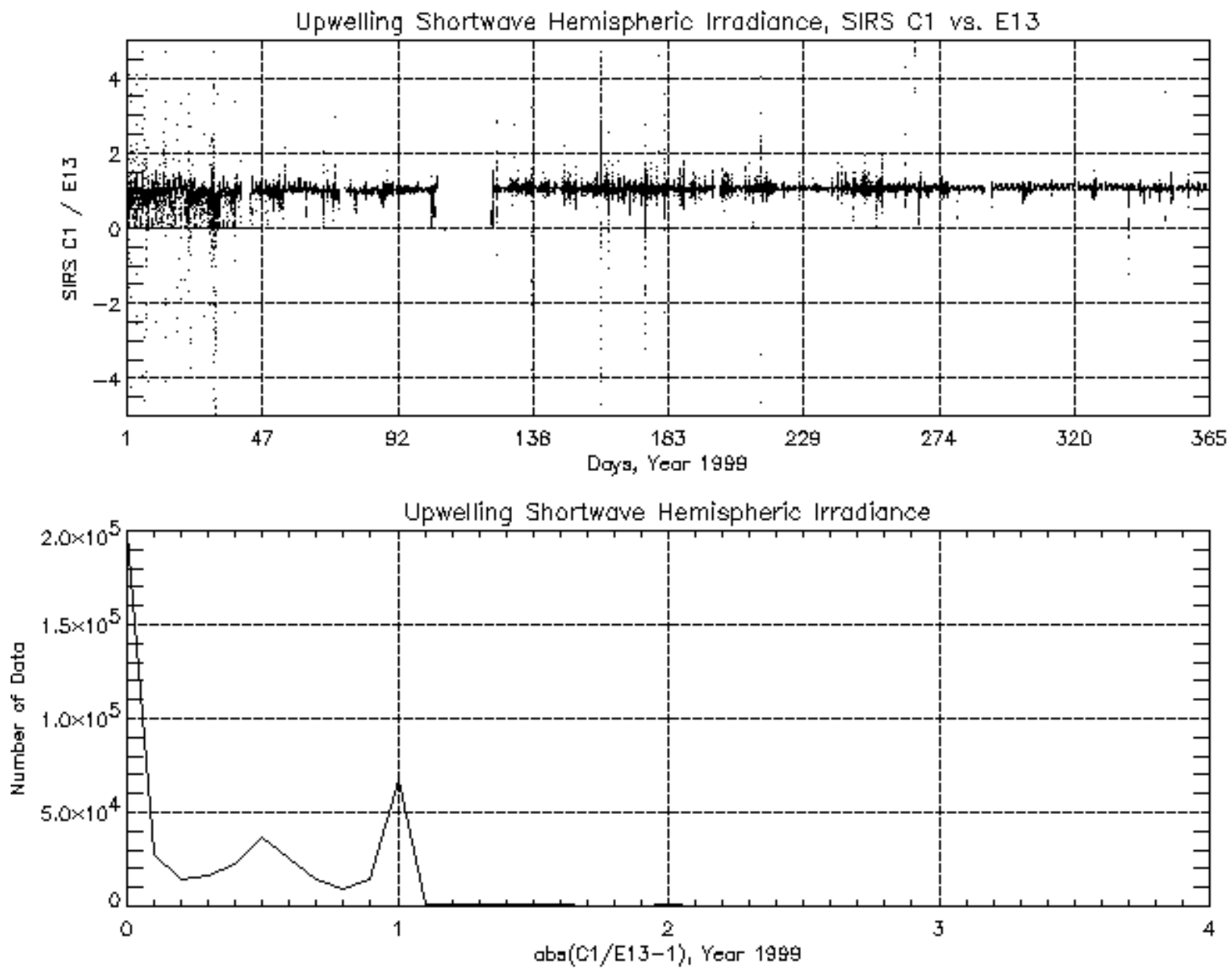

Figure 14. SIRS C1 vs. SIRS E13 Upwelling SW data for the year 1999. Top plot: the ratio of SIRS C1/E13 data; Bottom plot: probability distribution of the ratio. 

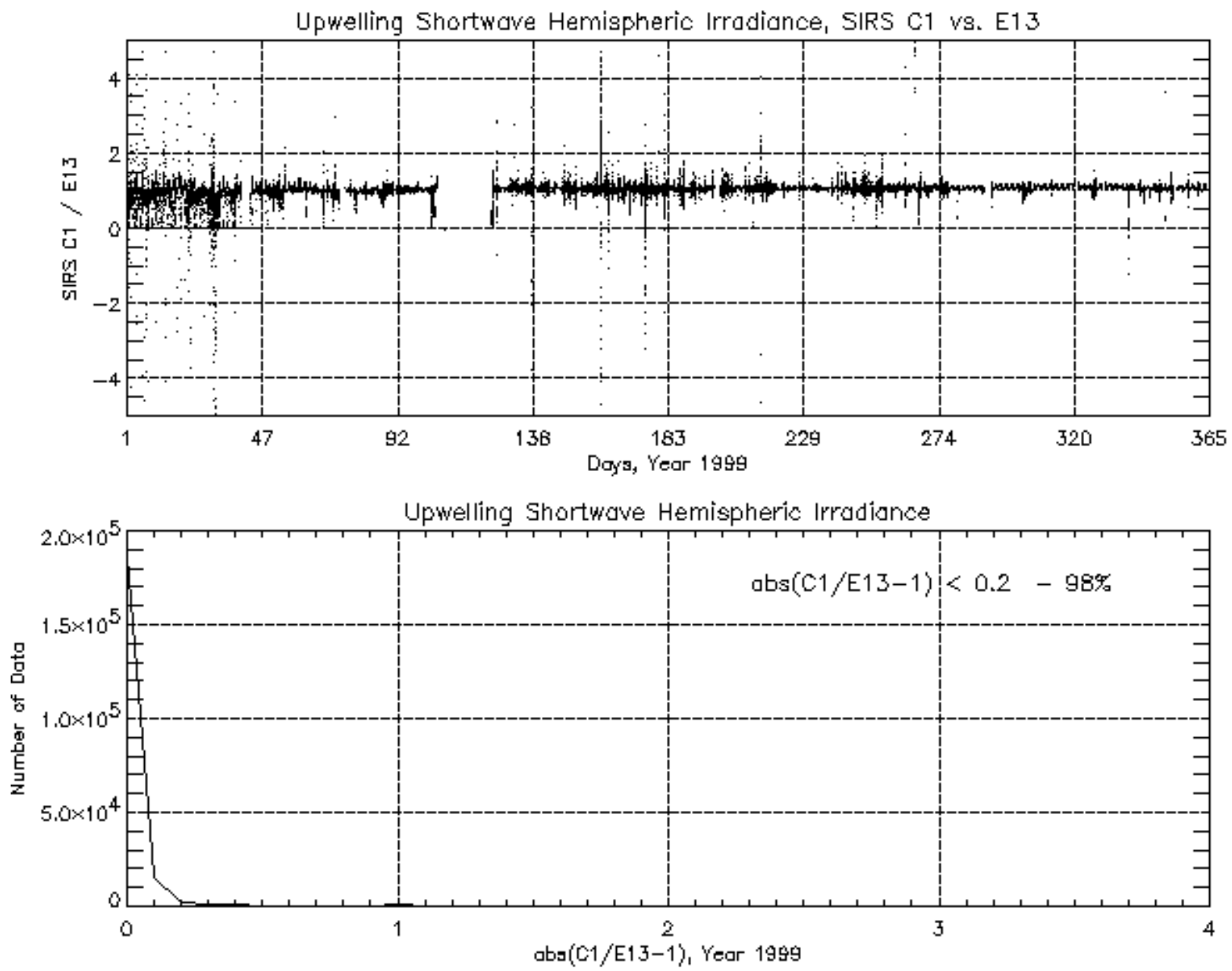

Figure 15. Same plot as Figure 14, but for SZA $<80^{\circ}$. 

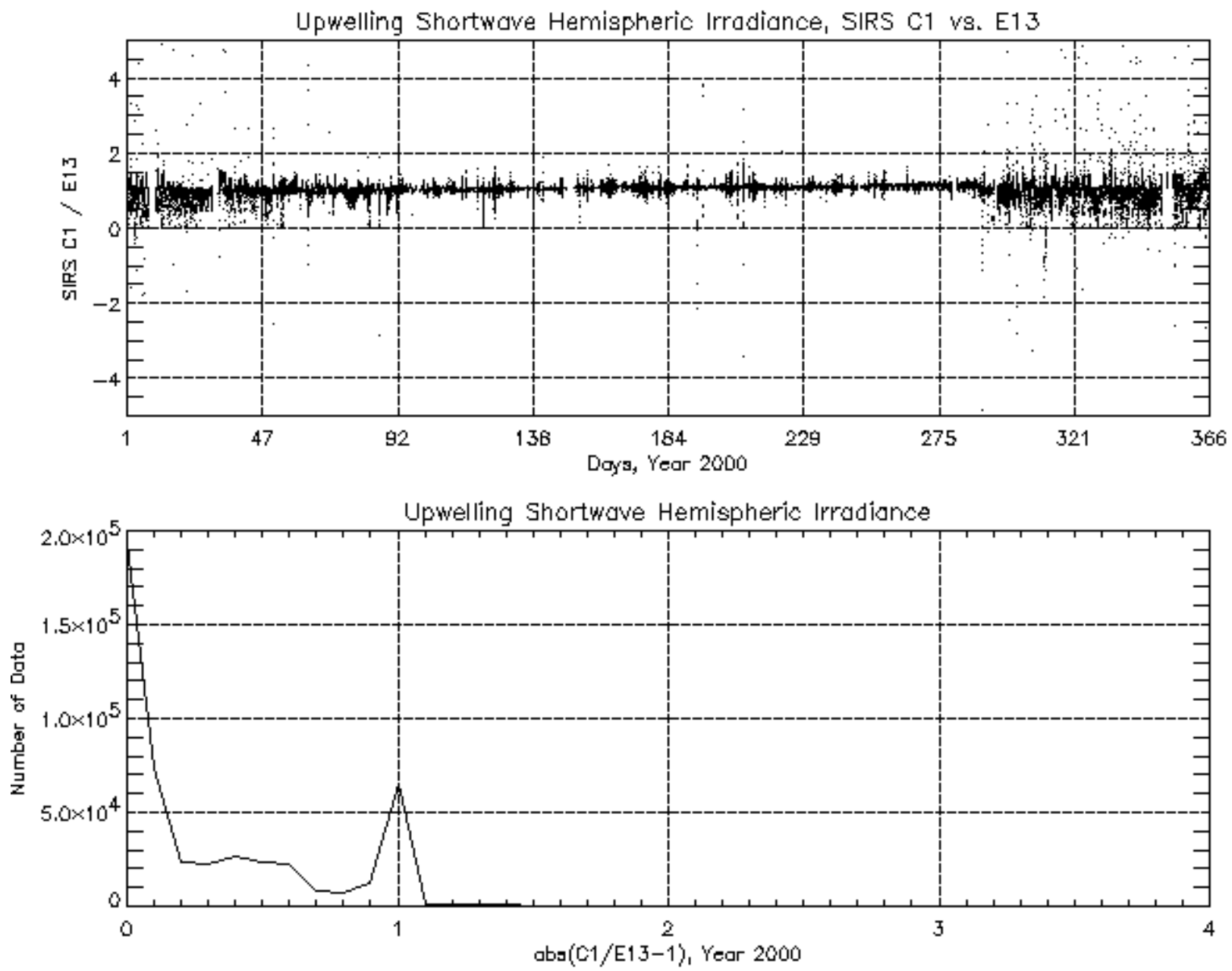

Figure 16. SIRS C1 vs. SIRS E13 Upwelling SW data for the year 2000. Top plot: the ratio of SIRS C1/E13 data; Bottom plot: probability distribution of the ratio. 

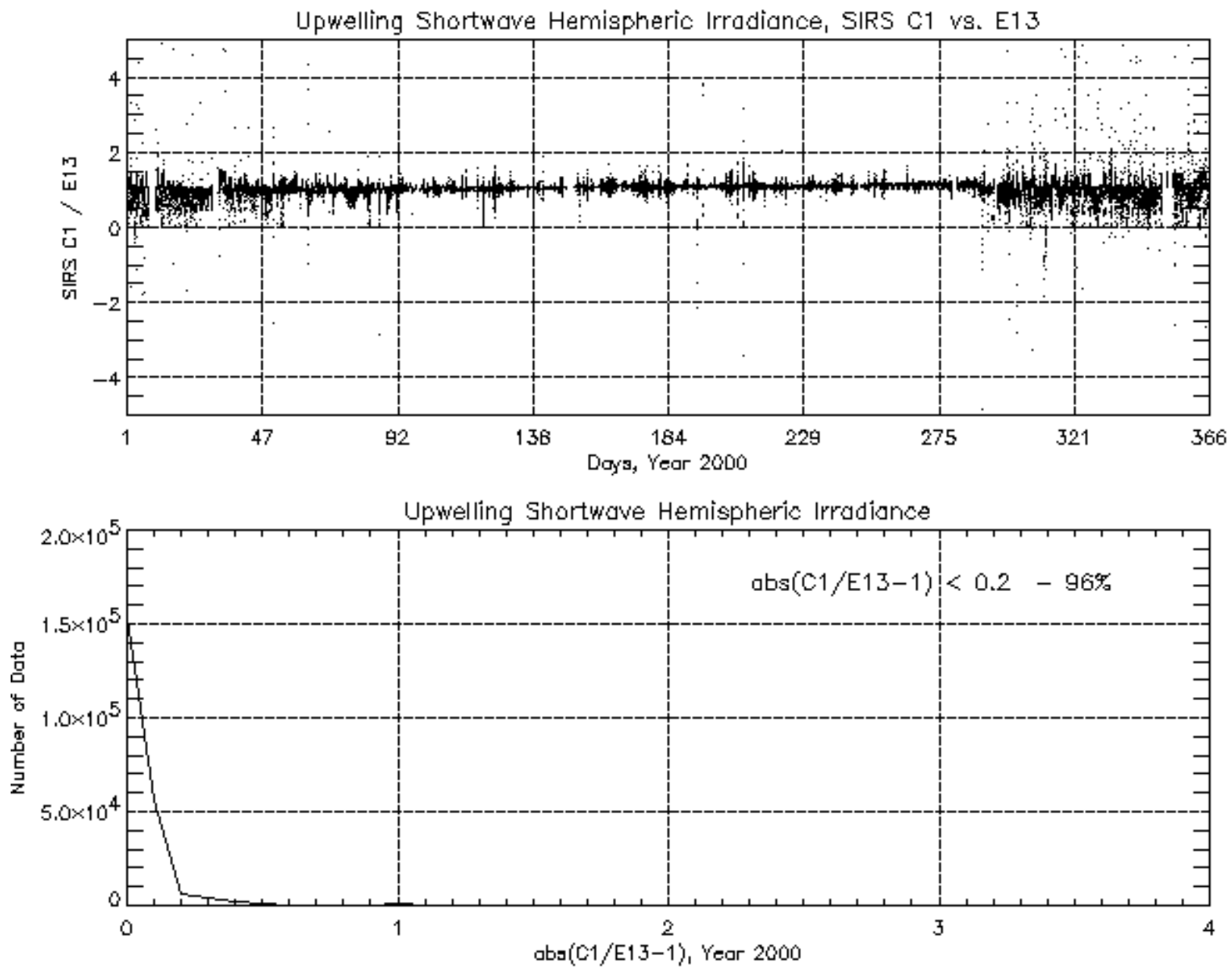

Figure 17. Same plot as Figure 16, but for SZA $<80^{\circ}$.

\subsection{Upwelling LW}

Figure 18 to Figure 20 are similar plots as Figure 2 to Figure 4, but for upwelling LW. Figure 18 shows that most data differences fall within 4\% in both 1999 and 2000. Figures 19 and 20 show the same thing except for the period of time in year 2000 (day 126 to day 216) where at least one instrument is not behaving well. Another thing to point out here is that in both years there is a sudden increase in the data difference at around day 220 then a gradual decrease until day 280 when it returned to "normal". This suggests an annual ground event happens at this time of the year (for example, the fall "browning" of the plants).

Figures 21 and 22 show the ratio of IRT flux vs. upwelling LW flux (ULF), for year 1999 and 2000, respectively. Here, we convert the IRT brightness temperature into flux using the Stephan-Boltzman relation, i.e., (flux $=\sigma \mathrm{T}_{\mathrm{b}}{ }^{4}$ ). We can see that the ratio of IRT flux over upwelling LW flux is a good indicator of the SIRS LW and IRT data agreement. Figure 22 clearly shows that something is wrong with SIRS C1 for the "bad data" period in year 2000, as shown in Figure 20. Therefore, we use the ratio as part of the criteria for best estimate calculation. Figures 23 and 24 are the upwelling LW vs. the IRT flux 
trend plots, for year 1999 and 2000, respectively. By visually inspecting the data, we found the data from May 9 to August 3, 2000, to be problematic. These data are marked as red in Figure 24. Aside from these "bad data", the results are very close for both instruments in both years; again indicating IRT flux is a useful tool for data quality determination.

Figure 25 shows the 15 -minute running standard deviation of the ratio $\sigma \mathrm{T}_{\mathrm{B}}{ }^{4} / \mathrm{ULF}$ for year 1999 . The upper plot is for SIRS C1 and the lower plot is for SIRS E13. Figure 26 is the frequency distribution of these standard deviations. We can see that for both instruments, the standard deviation falls mostly below 0.01 (98.0\% for SIRS C1 and 99.0\% for SIRS E13). Figures 27 and 28 are the same plots as Figures 25 and 26, but for year 2000. The period where SIRS C1 was problematic is shown clearly in the upper plot of Figure 27. This also accounts for the percentage drop shown in Figure 28 for SIRS C1, i.e., standard deviations below 0.01 are only $90.7 \%$, while for SIRS E13 99.9\% of the ratio data are below 0.01 .

In conclusion, we can use an irradiance data difference $<4 \%$ or an IRT flux - LW ratio standard deviation $\leq 0.01$ as criteria for upwelling LW flux data quality assessment.
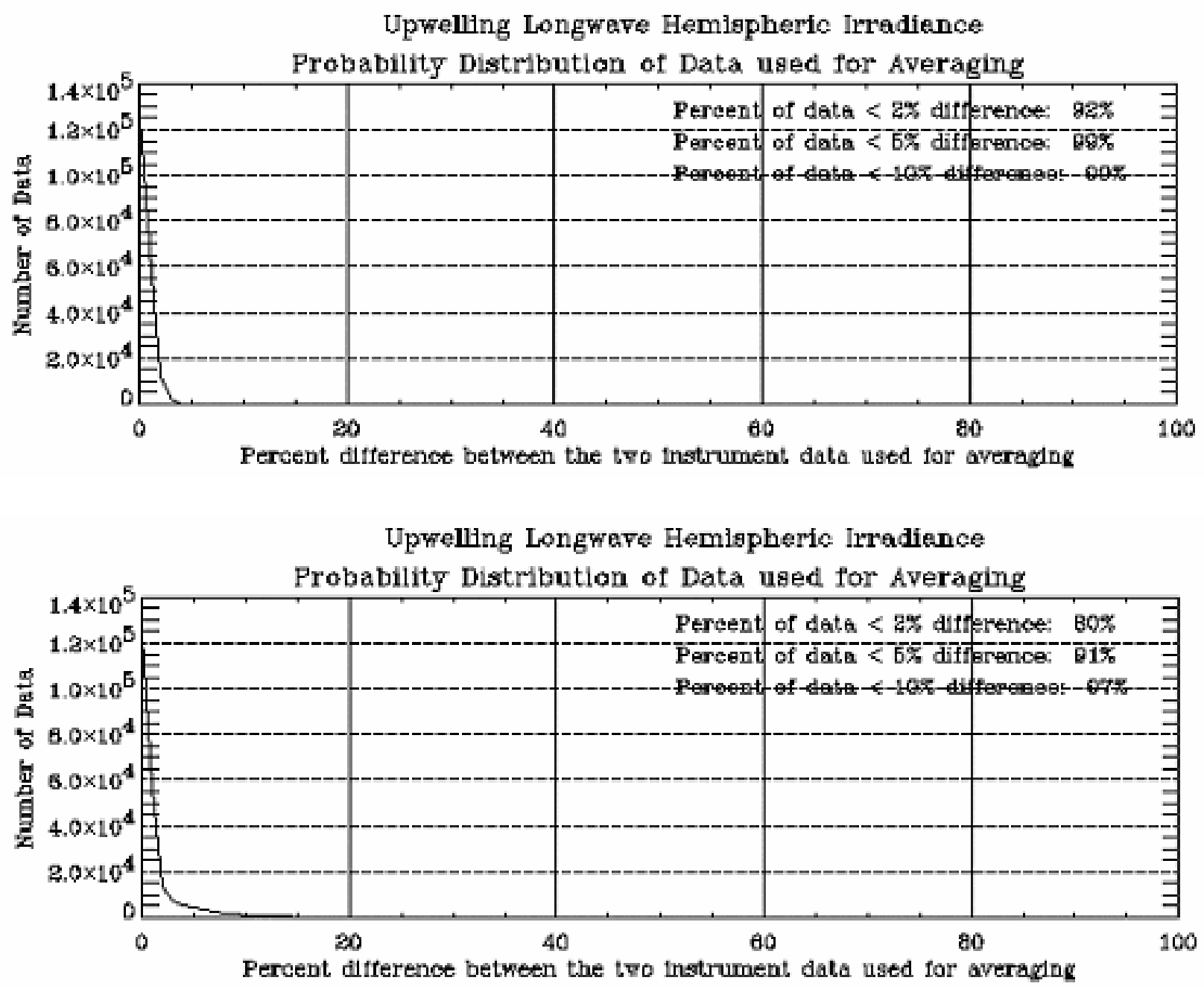

Figure 18. Probability distribution of upwelling LW percent differences for instrument pairs. Upper plot: year 1999; Lower plot: year 2000. 

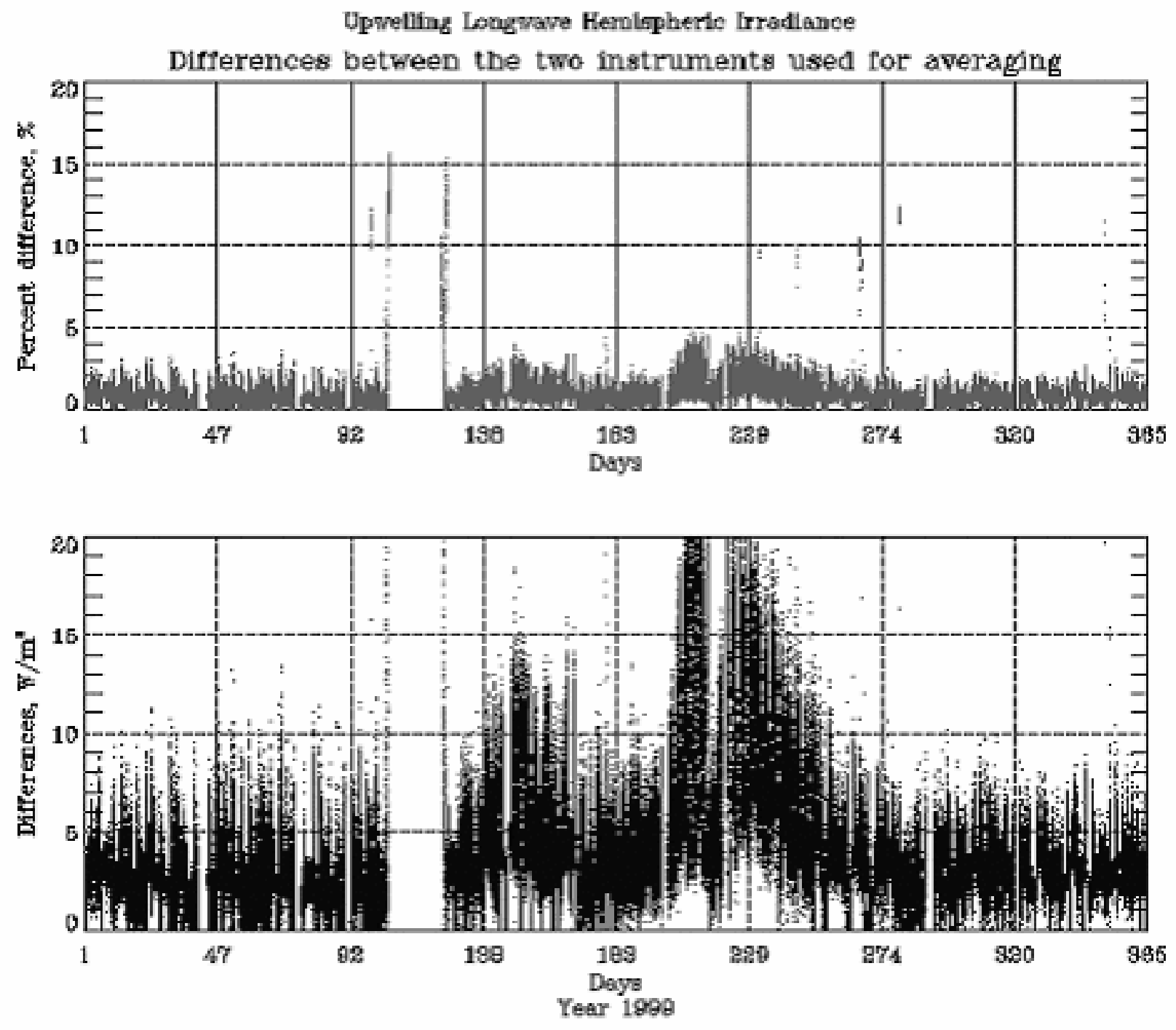

Figure 19. Differences of upwelling LW data in the year 1999. Upper plot: percent differences of the data with the SZA less than $80^{\circ}$, the data with the best estimate value greater than $20 \mathrm{Wm}^{-2}$ are colored red; Lower plot: absolute differences of the data with the SZA less than $80^{\circ}$, the data with the best estimate value less or equal to $20 \mathrm{Wm}^{-2}$ are colored blue. 
Upnelling Lobugave Hernlapherlo Irradlance

Differences between the two instruments used for averaging
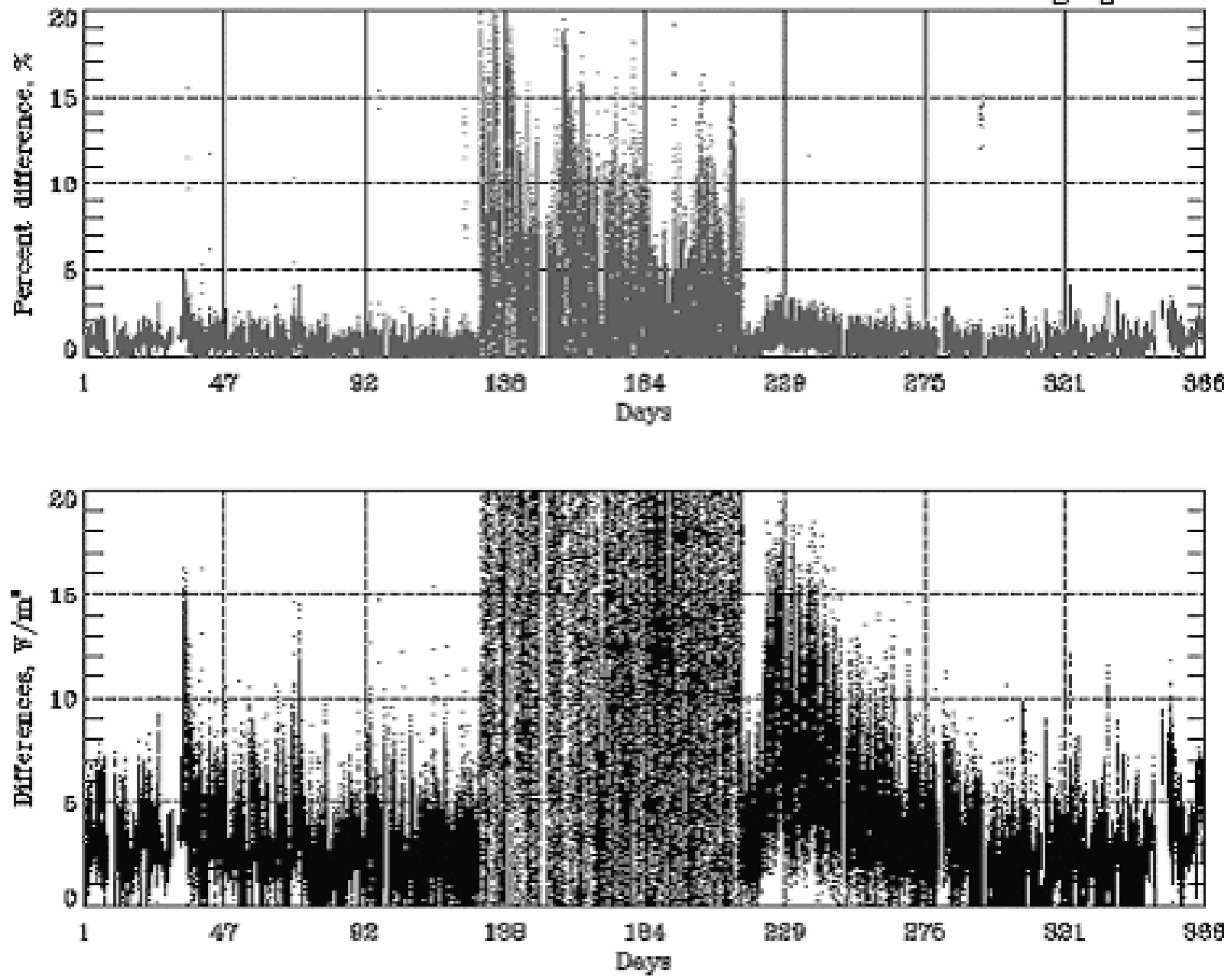

Figure 20. Same plot as Figure 19, but for the year 2000. 

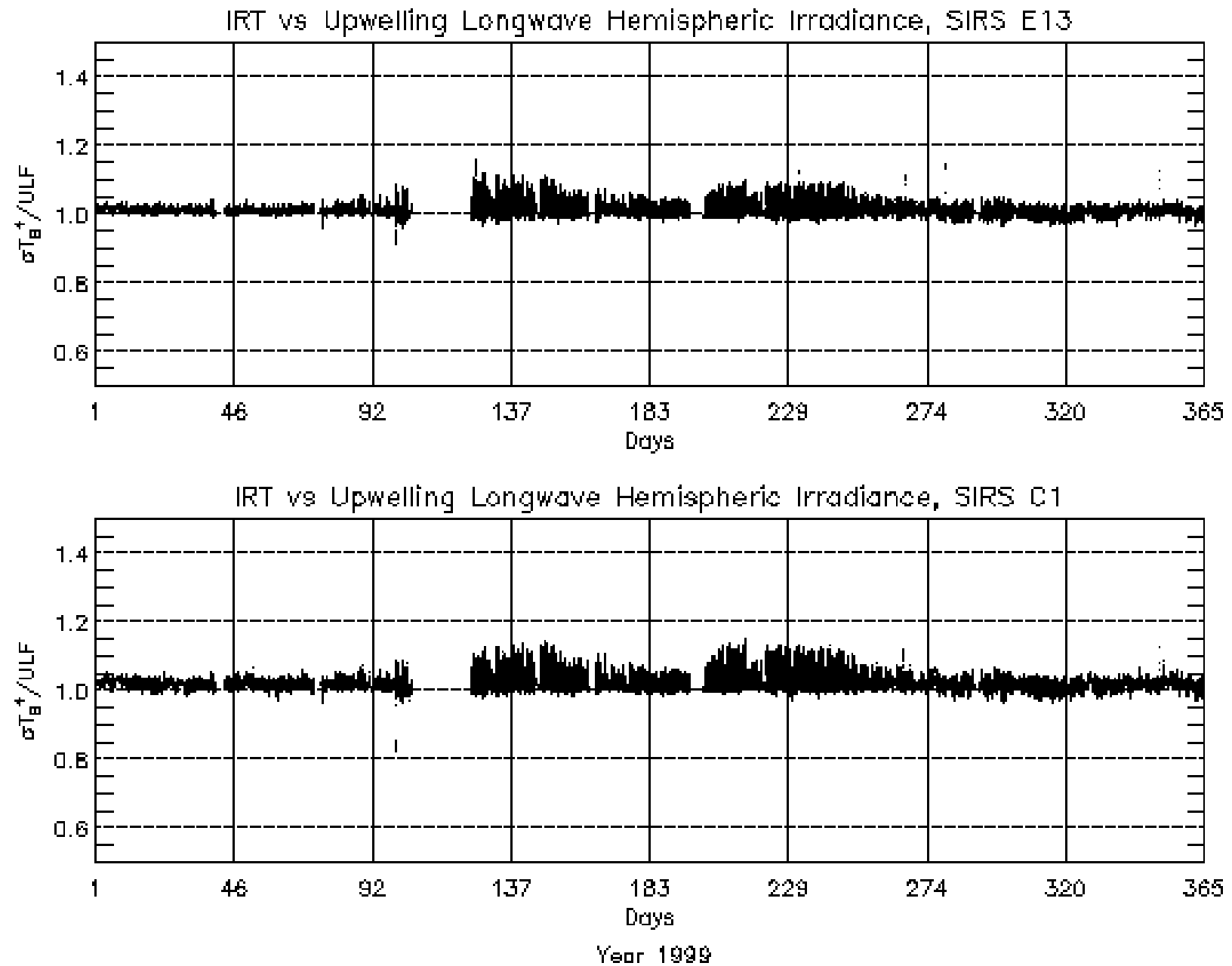

Figure 21. IRT flux vs. upwelling LW in the year 1999. Upper plot: SIRS E13 data; Lower plot: IRT flux vs. SIRS C1 data. 

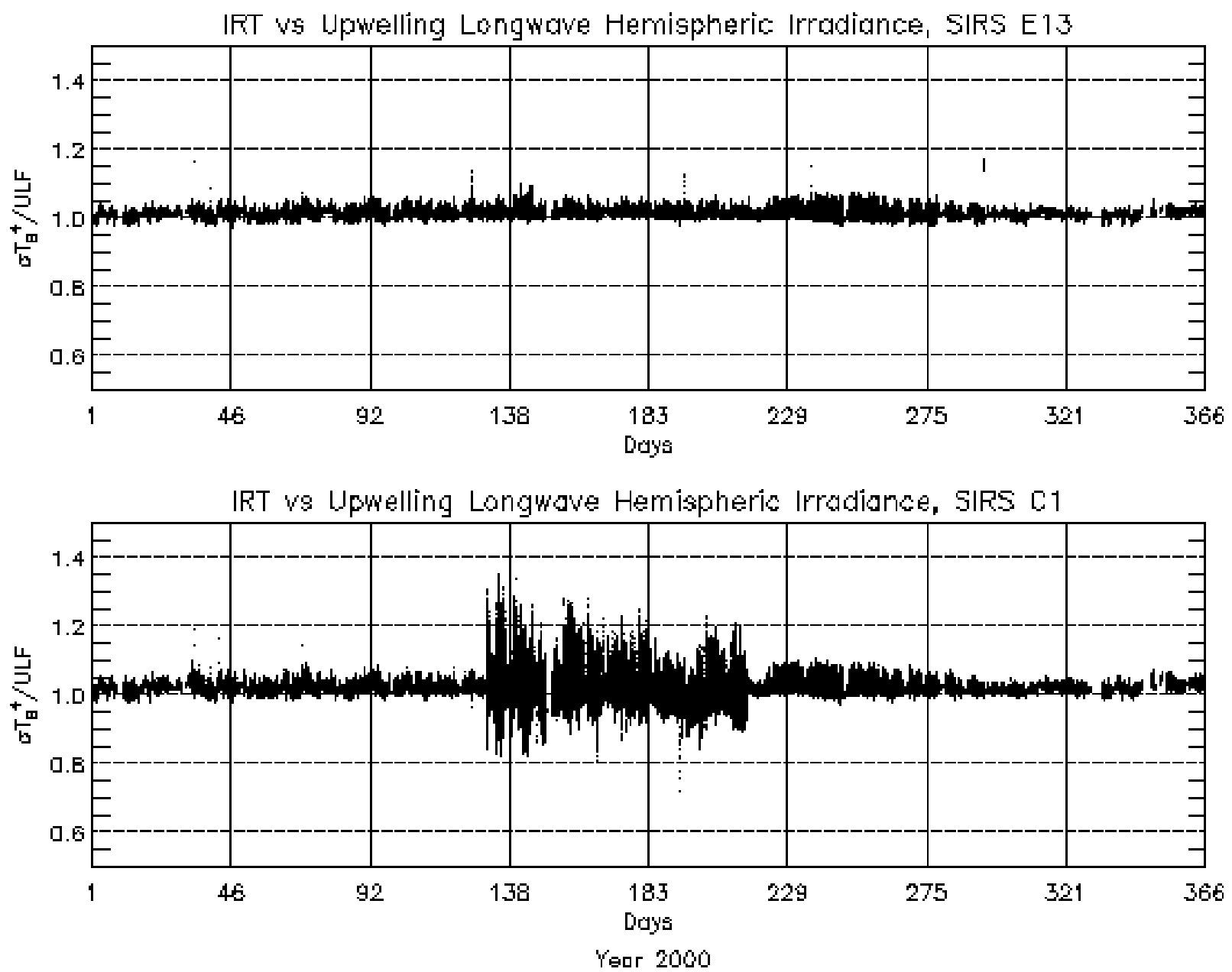

Figure 22. Same plot as Figure 21, but for the year 2000. 

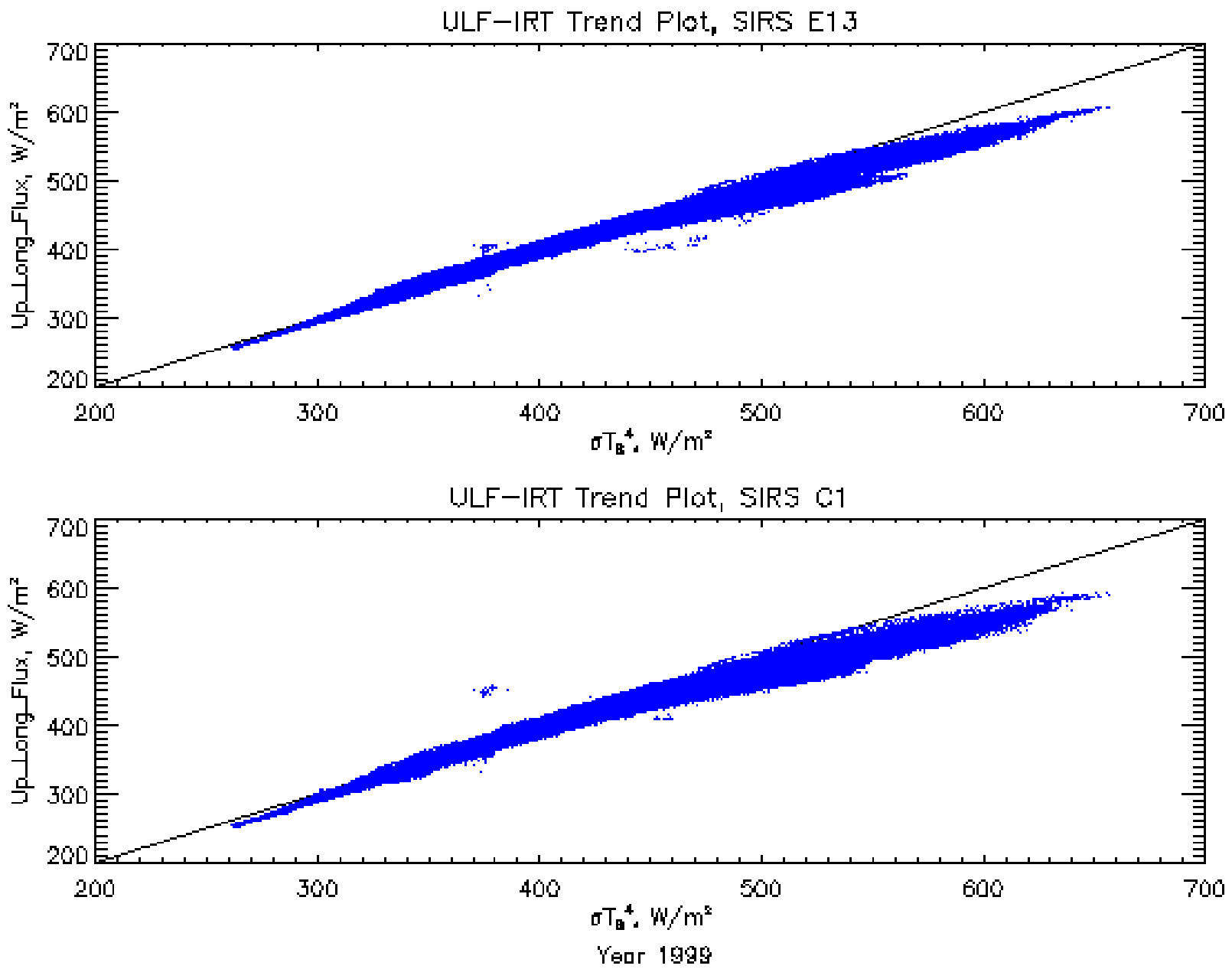

Figure 23. IRT flux vs. upwelling LW trend plot for the year 1999. 

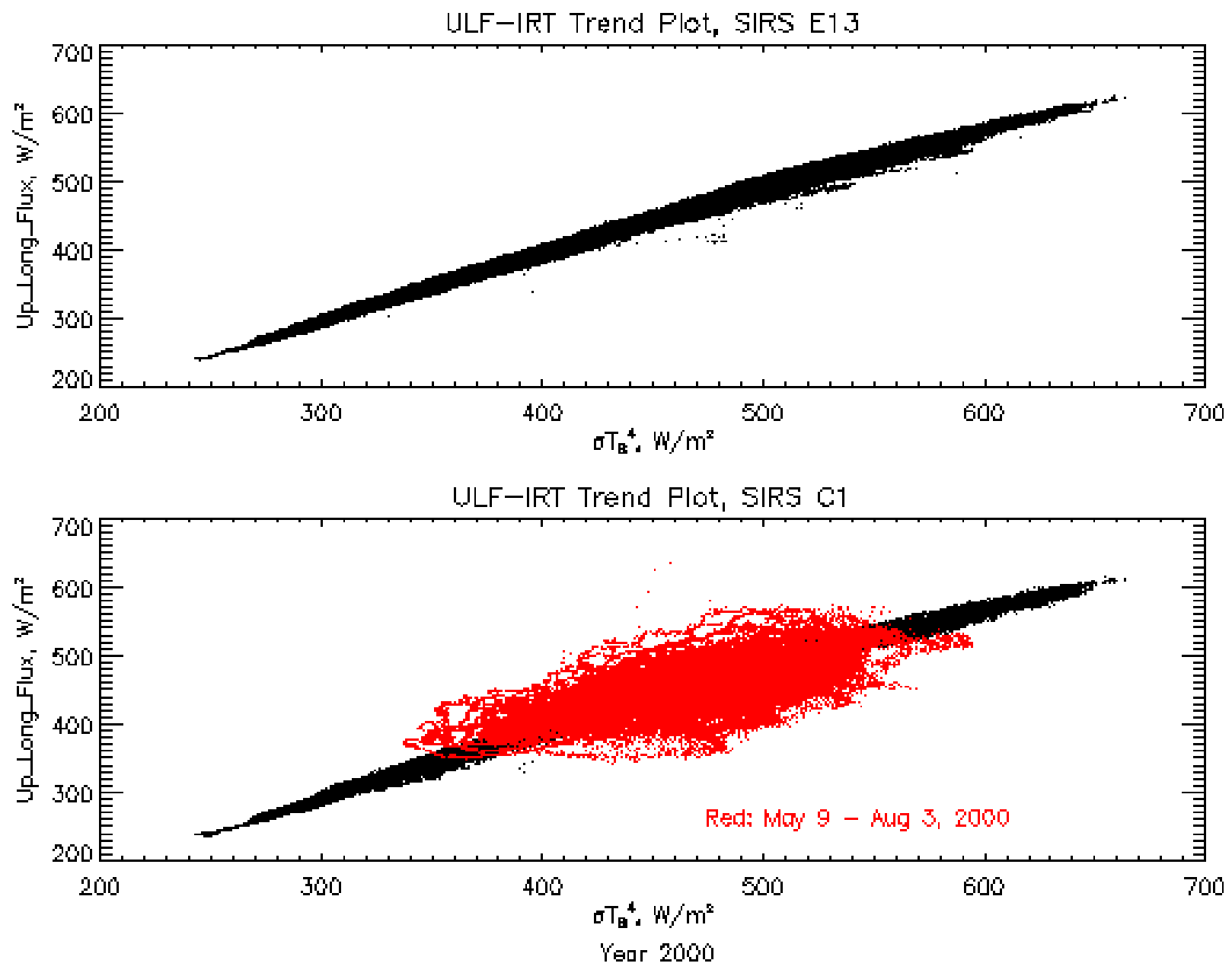

Figure 24. IRT flux vs. upwelling LW trend plot for the year 2000. 
15 Minute Running Standard Deviation of $\sigma \mathrm{T}_{\mathrm{B}}{ }^{4} / \mathrm{ULF}_{1}$ SIRS $\mathrm{C} 1$

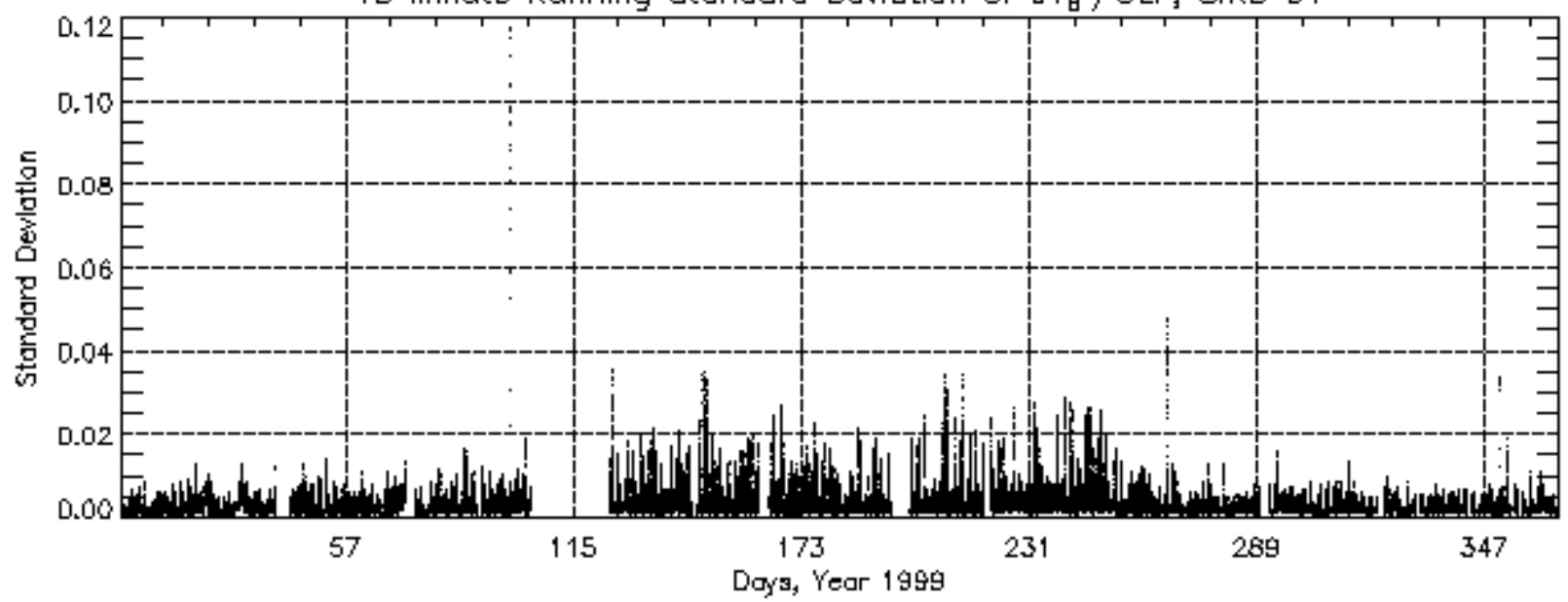

15 Minute Running Standard Deviation of $\sigma_{\mathrm{B}}{ }^{+} / \mathrm{ULF}$, SIRS E13

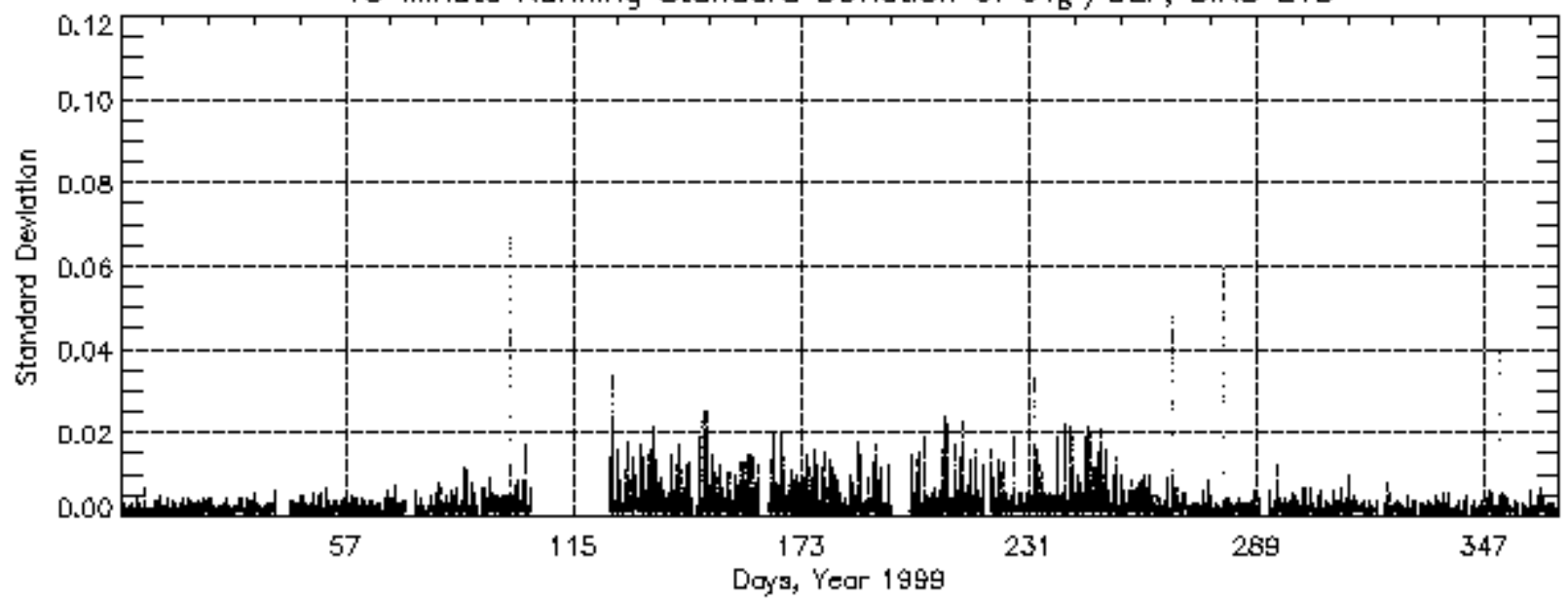

Figure 25. 15-minute running standard deviation of the ratio of IRT flux over upwelling LW in the year 1999. Upper plot: IRT Flux over SIRS C1 data; Lower plot: IRT flux over SIRS E13 data. 

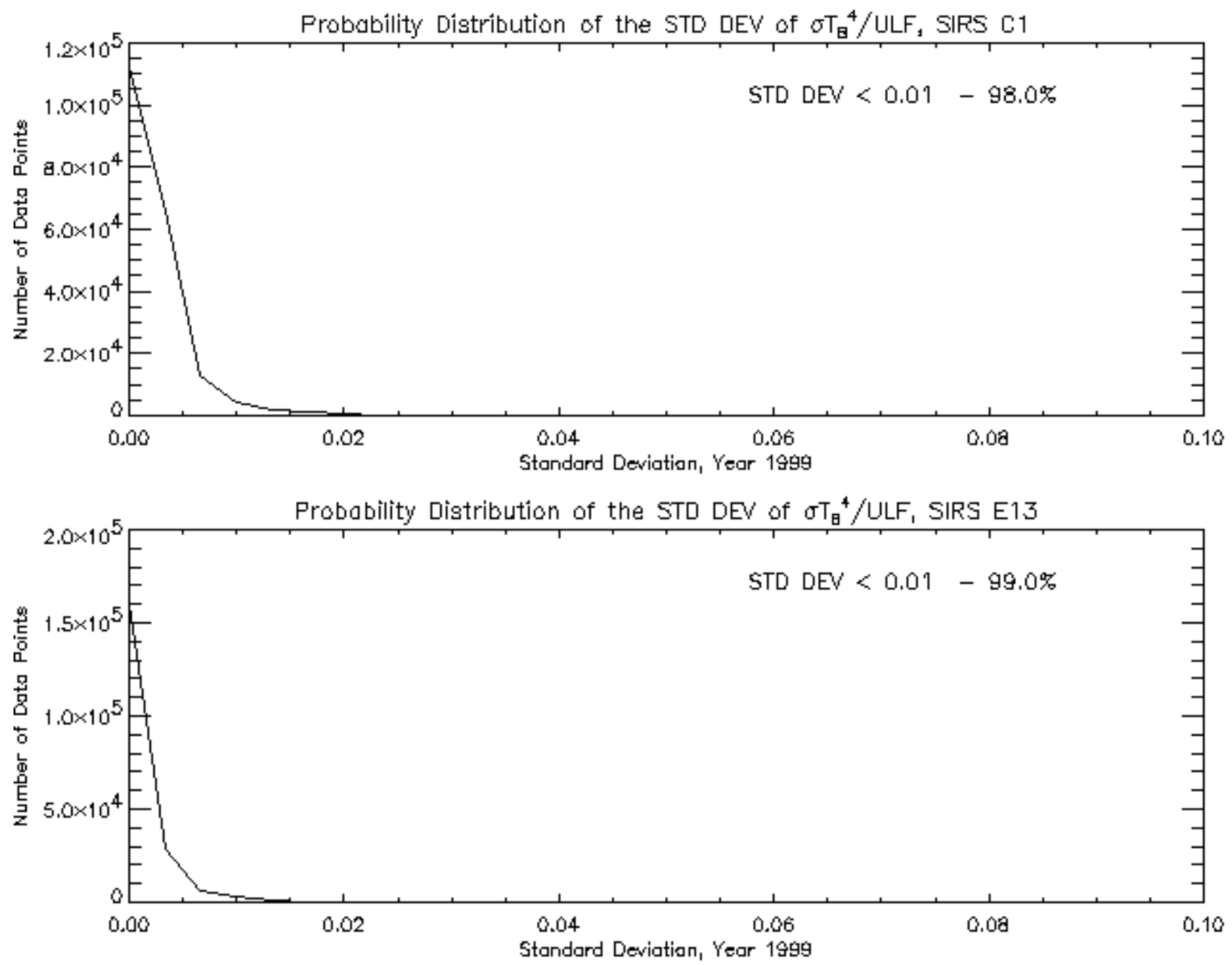

Figure 26. Frequency distribution of the standard deviation of the IRT flux vs. upwelling LW data in the year 1999. 
15 Minute Running Standard Deviation of $\sigma \mathrm{T}_{\mathrm{B}}{ }^{4} / \mathrm{ULF}_{1}$ SIRS $\mathrm{C} 1$

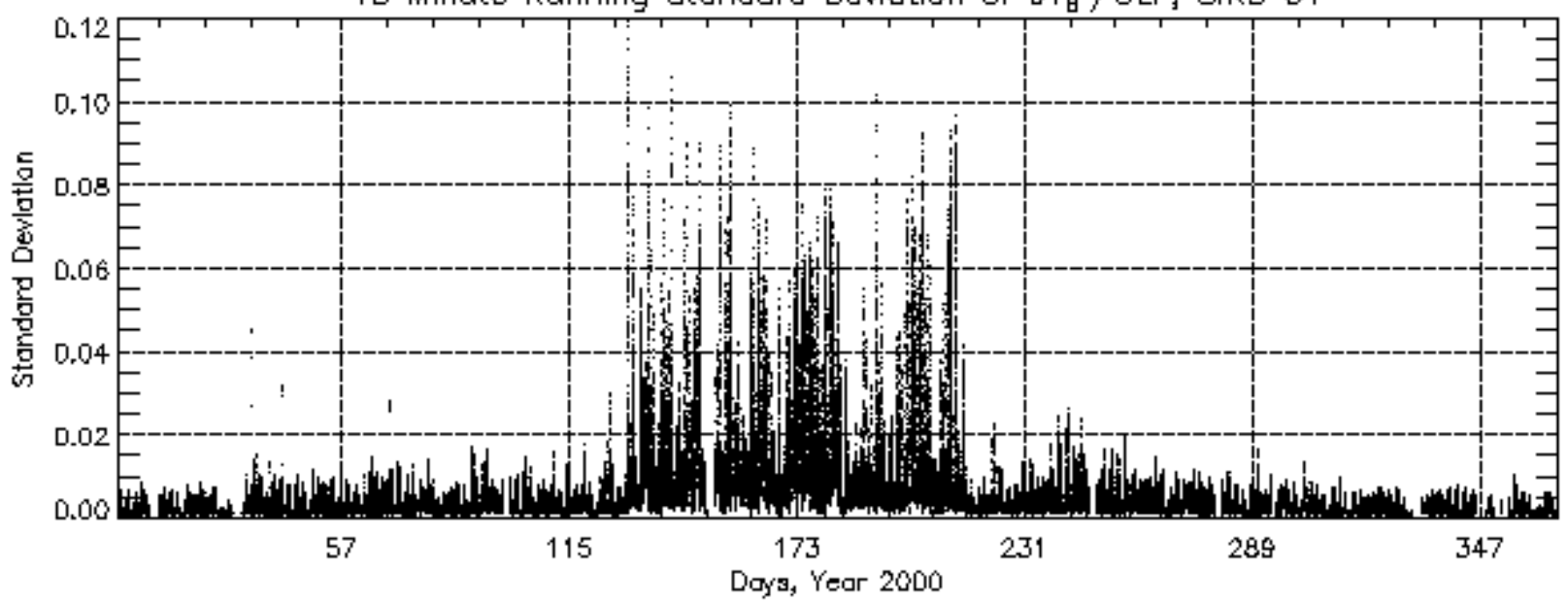

15 Minute Running Standard Deviation of $\sigma \mathrm{T}_{\mathrm{B}}^{+} / \mathrm{ULF}$, SIRS E13

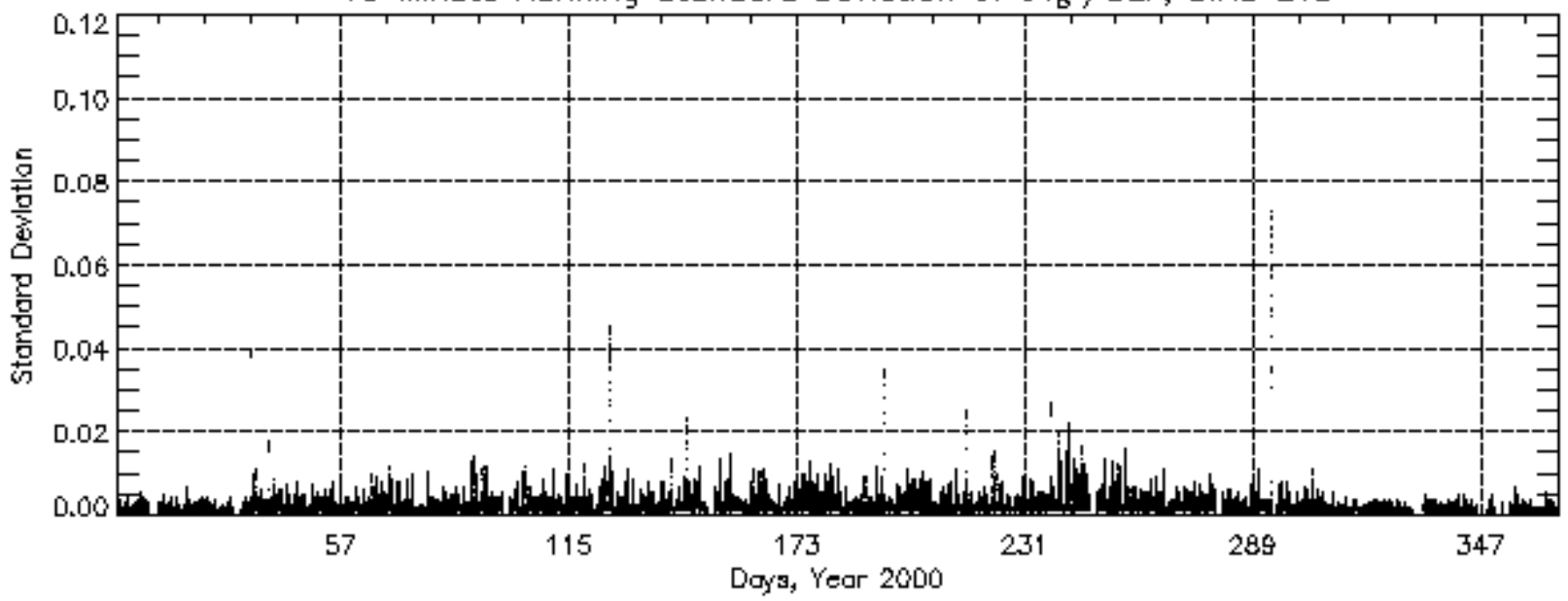

Figure 27. 15-minute running standard deviation of the ratio of IRT flux over upwelling LW in the year 2000. Upper plot: IRT Flux over SIRS C1 data; Lower plot: IRT flux over SIRS E13 data. 

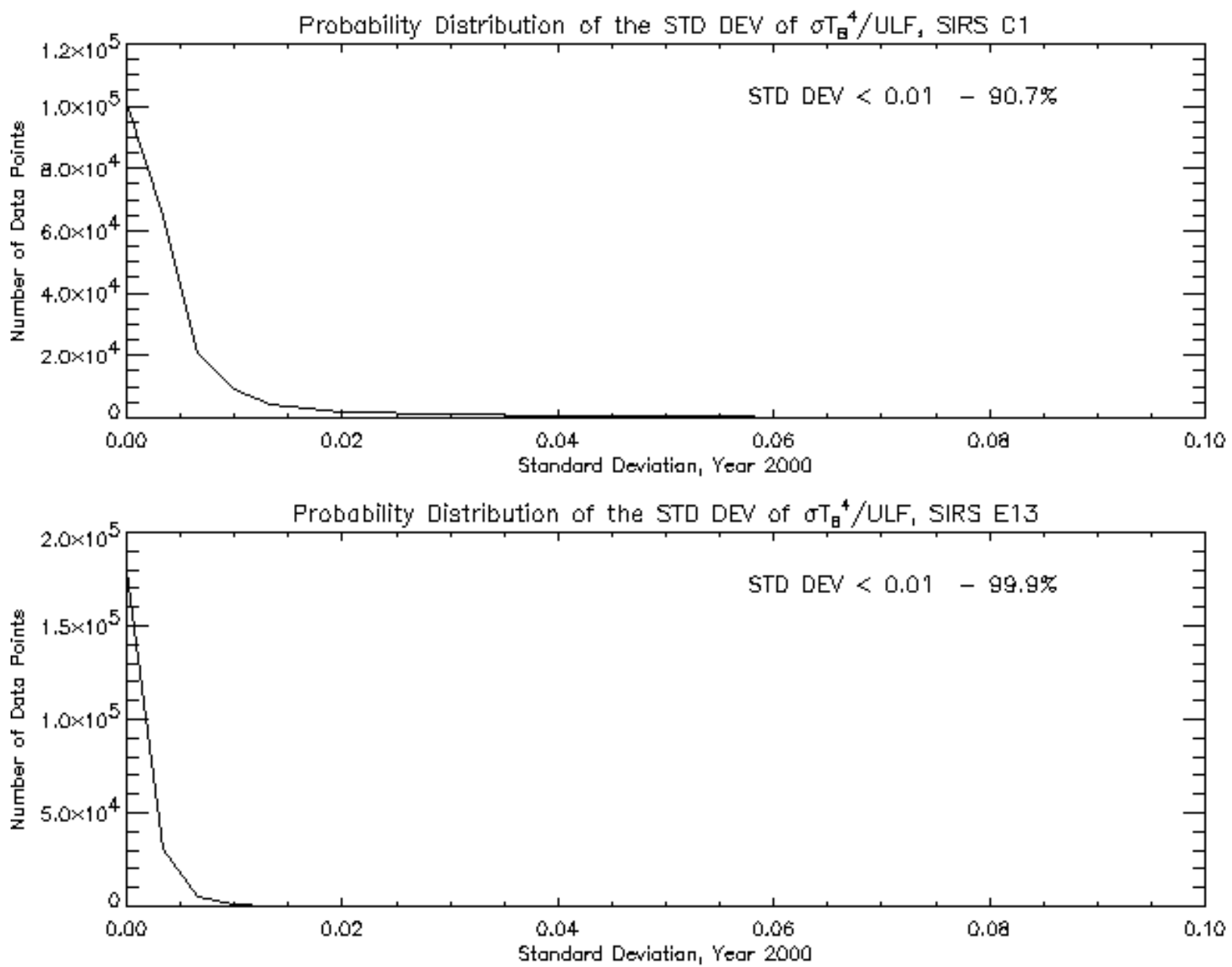

Figure 28. Frequency distribution of the standard deviation of the IRT flux vs. upwelling LW data in the year 2000.

\subsection{Analysis Summary}

Below is a summary table for the criteria/limits discussed in Sections 5.1 - 5.5 .

\begin{tabular}{|l|l||}
\hline \multicolumn{1}{|c||}{ Field } & \multicolumn{1}{c|}{ Criteria/Limits } \\
\hline Diffuse SW & Data difference $<10 \%$ or $5 \mathrm{Wm}^{-2}$, whichever is greater \\
\hline Direct Normal SW & Data difference $<5 \%$ or $5 \mathrm{Wm}^{-2}$, whichever is greater \\
\hline Downwelling LW & Data difference $<2 \%$ or $5 \mathrm{Wm}^{-2}$, whichever is greater \\
\hline Upwelling SW & $\begin{array}{l}\text { For data with SZA }<80^{\circ}, \text { abs }(\mathrm{C} 1 / \mathrm{E} 13-1)<0.2 \text {; for data with zenith angle }>=80^{\circ} \text {, data } \\
\text { difference }<10 \% \text { or } 5 \mathrm{Wm}^{-2}, \text { whichever is greater }\end{array}$ \\
\hline Upwelling LW & Data difference $<4 \%$ or IRT flux $-\mathrm{LW}$ ratio standard deviation $<0.01$ \\
\hline
\end{tabular}




\subsection{Case Studies}

In analyzing 1999 and 2000 data, the VAP detected some periods when data have apparent problems. As shown in Figure 29 for year 2000 data, the top plot shows the best estimate of downwelling diffuse SW with periods when SIRS C1 data was not used in the best estimate calculation colored red. The bottom plot is the difference between the best estimate value and each instrument data. The top plot indicates SIRS C1 data is thrown out during a period between day 92 and day 138. The bottom plot shows SIRS C1 data switched signs during this time period. By examining the monthly data we confirmed SIRS C1 B/W data stream switched signs starting from April 24, around 2000 hours, until May 9, at about 1700 hours. The same problem is also found in direct normal SW, as shown in Figure 30. To investigate this time period, we obtained Data Quality Reports for the SIRS C1 data stream. DQR-D000919.1 indicates two wires were crossed during this time period, thus, producing negative values. This finding is a good indication that the VAP is working properly, throwing out the problematic data as expected.
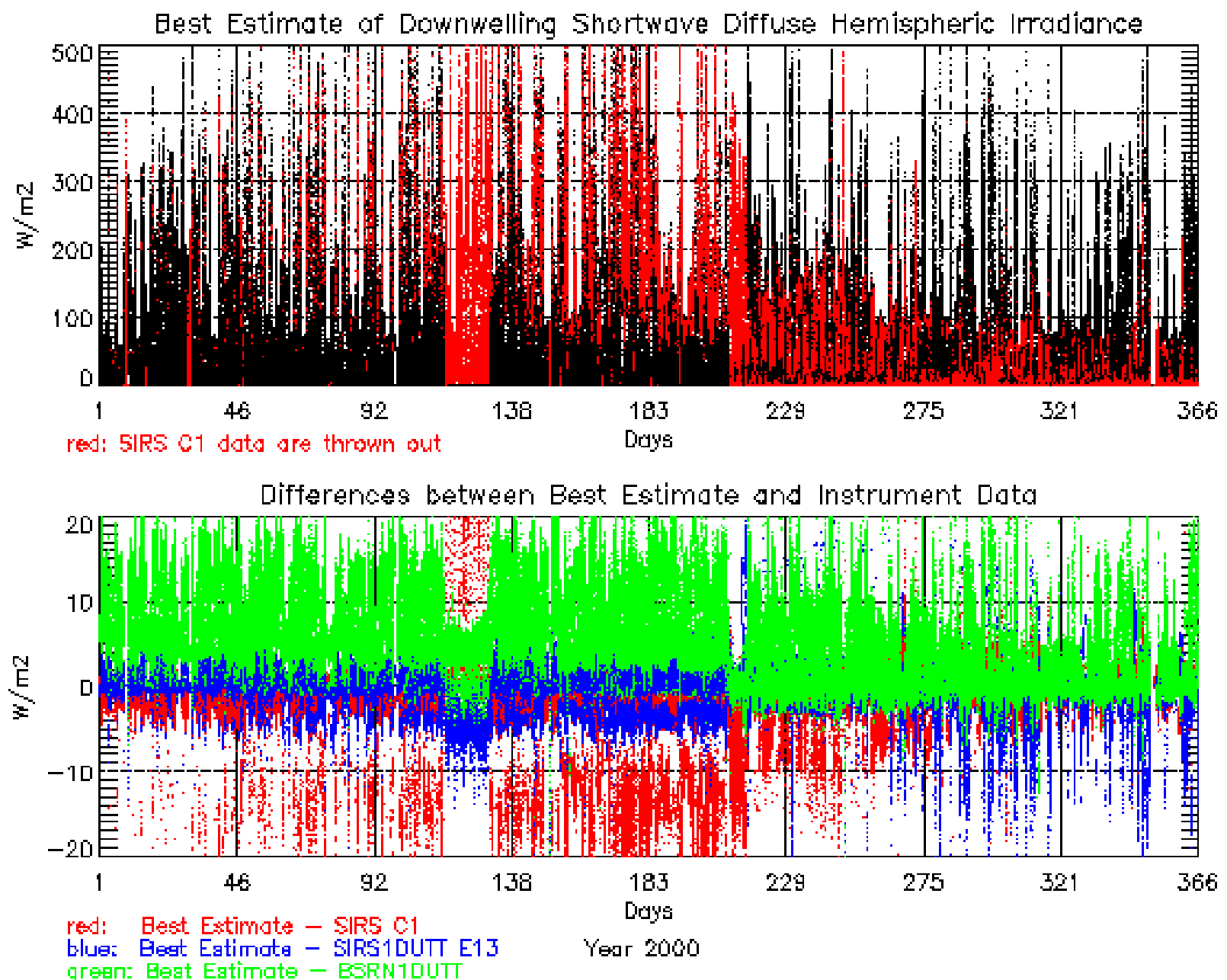

Figure 29. Best estimate of downwelling diffuse SW (top plot) and the differences between best estimate and instrument data (bottom plot) in the year 2000. In the top plot, the red curves mark the instances when SIRS C1 data was not used in the best estimate calculation. In the bottom plot, the red, blue, and green colors represent the differences of the best estimate value and the SIRS C1, E13, and BRS data, respectively. 

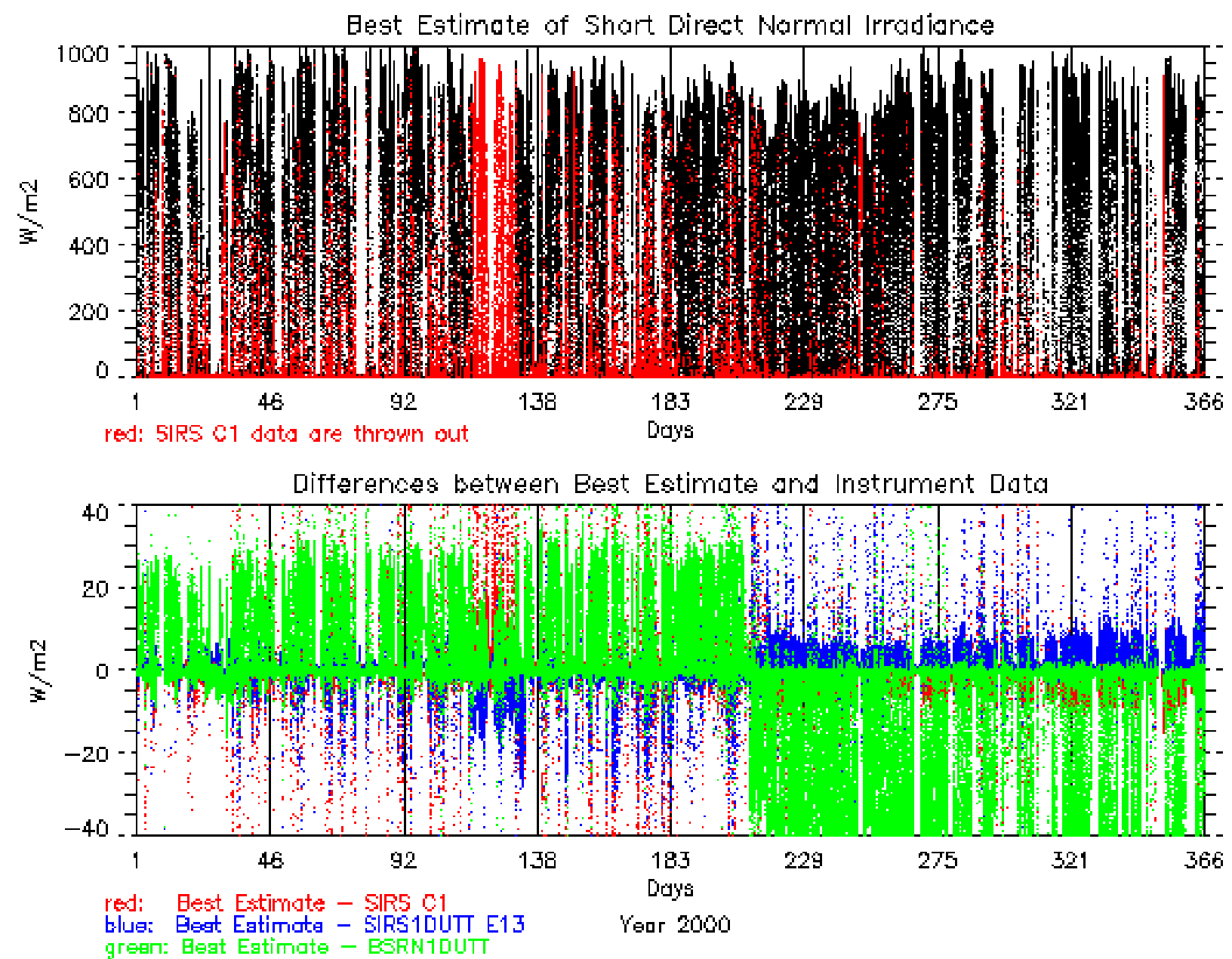

Figure 30. Best estimate of direct normal SW (top plot) and the differences between best estimate and instrument data (bottom plot) in the year 2000. In the top plot, the red curves mark the instances when SIRS C1 data was not used in the best estimate calculation. In the bottom plot, the red, blue, and green colors represent the differences of the best estimate value and the SIRS C1, E13, and BRS data, respectively.

\section{Data Qc Tests and Qc Flags}

\subsection{Input Data Availability}

Figure 31 shows the percent of possible data available during daytime for SIRS E13, SIRS C1, and BRS spanning the years 1997 to 2001 (i.e., all the data not equal to -9999 from the input netCDF files). For SIRS E13 (blue curve), the data availability varies from $94.5 \%$ in 1997 to $99.7 \%$ in 2000 . For SIRS C1 (red curve), the data availability ranges from $93.9 \%$ in 1999 to $99.4 \%$ in 2001 . BRS data (green curve) has the least data available, ranging from $82.7 \%$ in 2001 to $98.7 \%$ in 2000. 


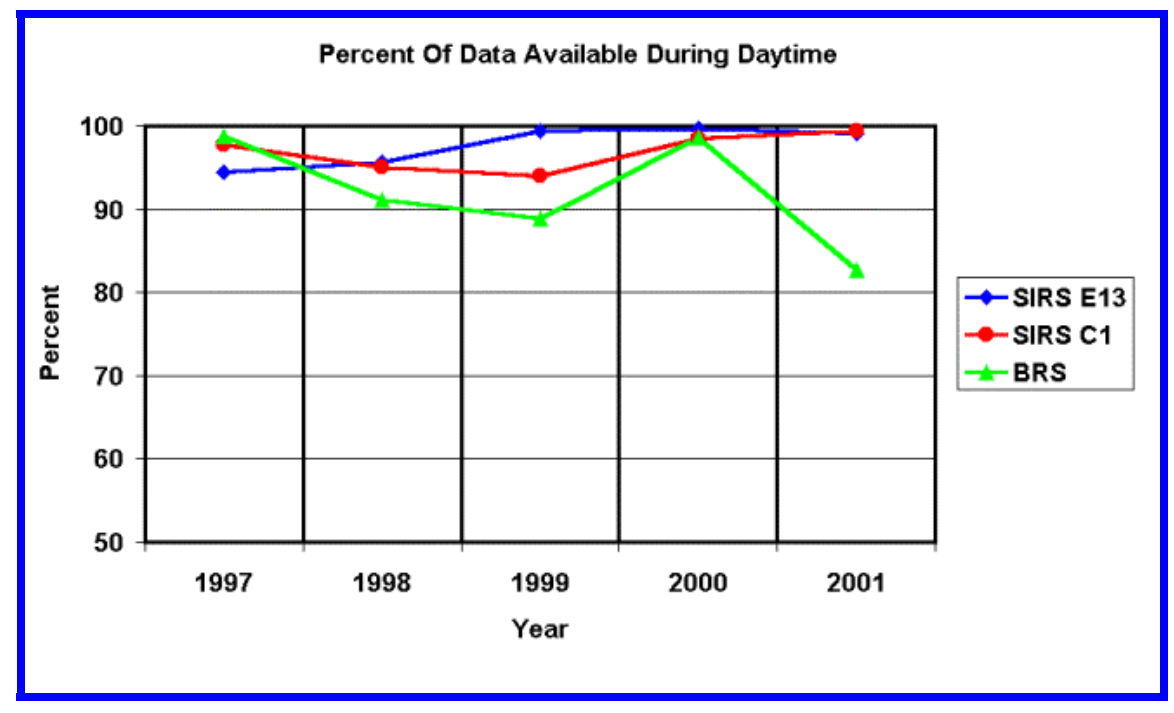

Figure 31. Percent of input data available by year and platform.

\subsection{Data Qc Tests and Qc Flags}

The ultimate goal of the BE Flux VAP is to automatically apply data quality checks to all the input data, exclude "bad" data, and decide which are the proper data to use for best estimate evaluation. The criteria/limits applied to each field are discussed in Section 5. What follows are the various values of the included data QC flags and a brief description of their meanings:

For the direct normal and diffuse SW, and downwelling LW:

$\begin{array}{ll}\text { Flag }=2 & \text { Average of E13 and C1 } \\ \text { Flag }=1 & \text { Average of BRS and C1 } \\ \text { Flag }=0 & \text { Average of BRS and E13 } \\ \text { Flag }=-1 & \text { BRS only } \\ \text { Flag }=-2 & \text { E13 only } \\ \text { Flag }=-3 & \text { C1 only } \\ \text { Flag }=4 & \text { Not enough info. } \\ \text { Flag }=-4 & \text { All instruments are down }\end{array}$

For the upwelling SW and LW:
Flag $=0$
Average of E13 and C1
Flag $=1$
E13 only
Flag $=2$
C1 only
Flag $=4 \quad$ Not enough info.
Flag $=-4 \quad$ All instruments are down 


\section{Output Files}

The SW Flux Analysis VAP produces two primary sets of 1-minute resolution output files: one contains best estimate of downwelling SW direct and diffuse, downwelling LW, upwelling SW and LW irradiances, the net surface radiation and the broadband SW surface albedo, along with the corresponding QC flag for each field, and the differences between the two instrument data used for averaging. The other file contains the above irradiance fields and the differences between the best estimate and each instrument data.

The BE Flux VAP produces two output files:

sgpbeflux1longC1.c1.YYYYMMDD.hhmmss.cdf

sgpqcflux1longC1.c1.YYYYMMDD.hhmmss.cdf

where

YYYY is the year, MM is the month of the year, DD is the day of the month, hh is the hour of the day of data start, $\mathrm{mm}$ is the minutes of the hour, and ss is the seconds of the minute. The output fields and their units and descriptions are listed in Section 3.

\section{Results}

Figure 32 shows the best estimate and differences between the best estimate and instrument data for the diffuse SW in the year 2000. The top plot is the best estimate and the bottom plot is the differences between the best estimate and instrument data. For the diffuse SW, three measurements from SIRS E13, $\mathrm{C} 1$, and BRS were used in the best estimate evaluation. In the bottom plot, the red curve is the differences between the best estimate and SIRS C1 data, the blue curve is the differences between the best estimate and SIRS E13 data, and the green curve shows the differences between the best estimate and BRS data. Note: SIRS1DUTT E13 and BSRN1DUTT marked on the plot indicate diffuse corrected data were used for platforms SIRS E13 and BRS.

Figure 33 shows the same plot as Figure 31, but for the direct normal SW. The bottom plot of Figure 32 indicates that BRS data is much lower than the best estimate data before day 210 and higher after day 210. Figure 34 shows the direct normal SW measured at the three instruments (SIRS E13, shown as black * in the plot; SIRS C1, shown as green +; and BSRN, shown as red x) from day 207 to 213. It is apparent from the plot that BRS data significantly differs from the data from the other two instruments. This indicates that something is wrong with the BRS measurement and that it should not be used in the best estimate calculation. The bottom plot of Figure 8.2 shows that the best estimate algorithm we used detected this successfully and that the BRS data was not used in the calculation of the best estimate.

Figure 35 shows the same plot as Figure 33, but for the downwelling LW. The top plot shows the typical seasonal variations of the downwelling LW, with higher irradiances in the summer months (days 180 250). The absolute differences among instruments are also higher compared to winter months, but the percentage of differences remains low, as shown in the top plot of Figure 10 in Section 5. 
Figure 36 is the same plot as Figure 35, but for upwelling SW. Since only two measurements from SIRS E13 and C1 are involved in the best estimate calculation of upwelling SW, the difference plot (bottom plot of Figure 8.5) shown here is the differences between these two instruments. The decrease of the upwelling SW irradiances at the end of the summer (around day 280) is probably due to the fall "browning" of the plants.

Figure 37 is the same plot as Figure 36, but for upwelling LW. The top plot of this figure clearly shows the seasonal variations of the upwelling LW. The bottom plot shows that the two instruments agree quite well most of the time during the year except for a period from around day 130 to 220 . The period was when SIRS C1 instrument was not behaving well, as discussed in Section 5.5.

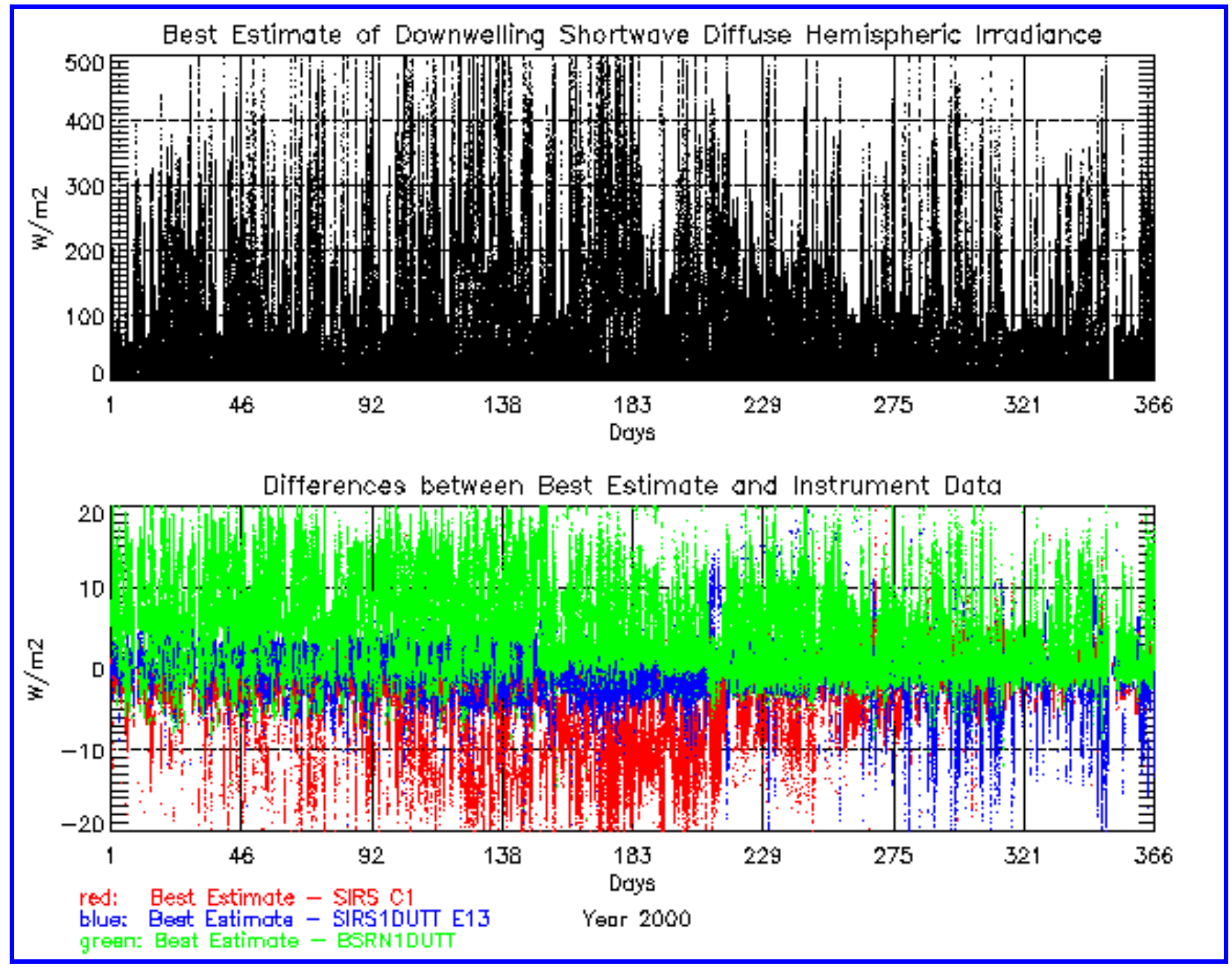

Figure 32. Best estimate of diffuse SW (top plot) and differences between best estimate and instrument data (bottom plot) in the year 2000. 


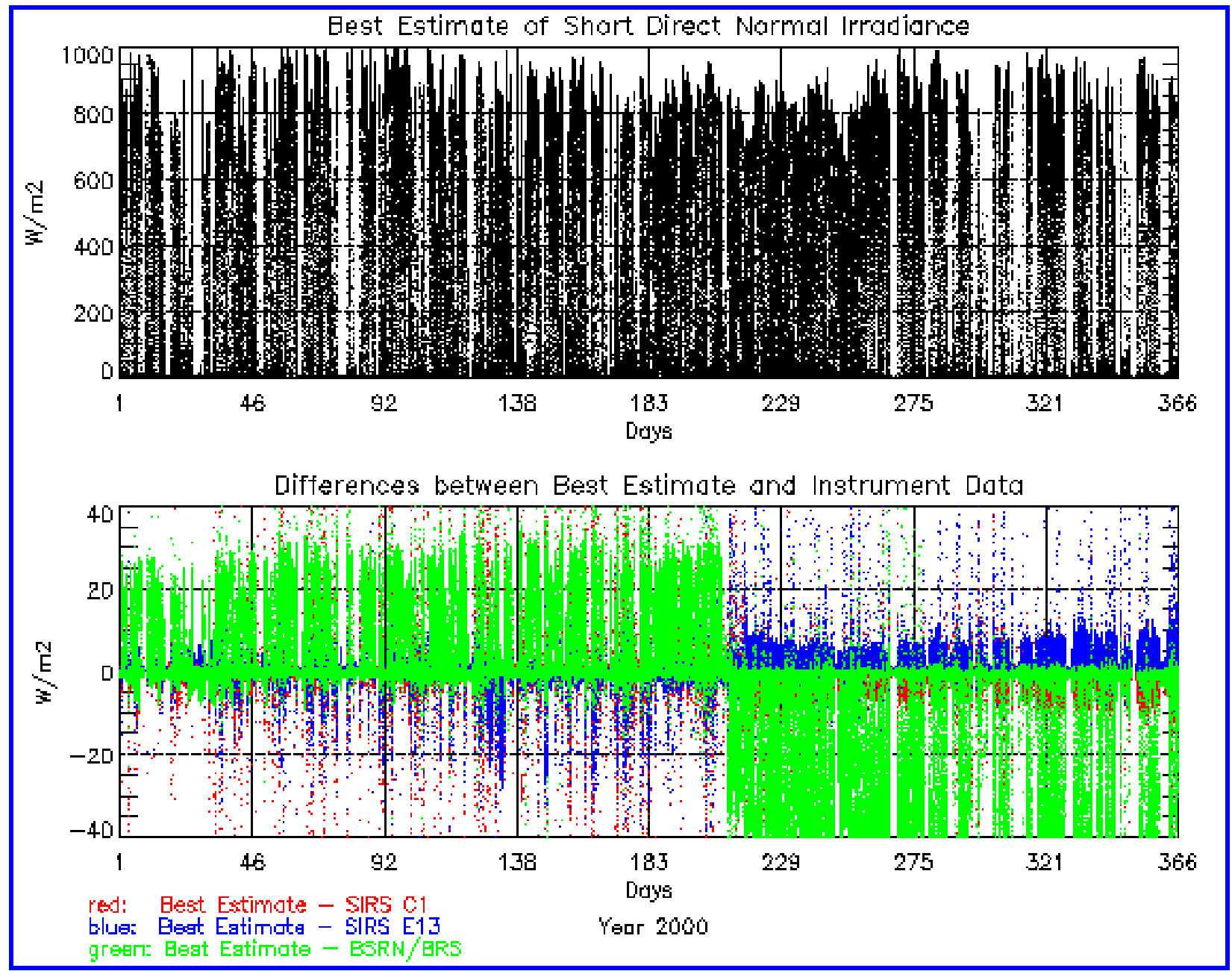

Figure 33. Same as Figure 32, but for direct normal SW. 


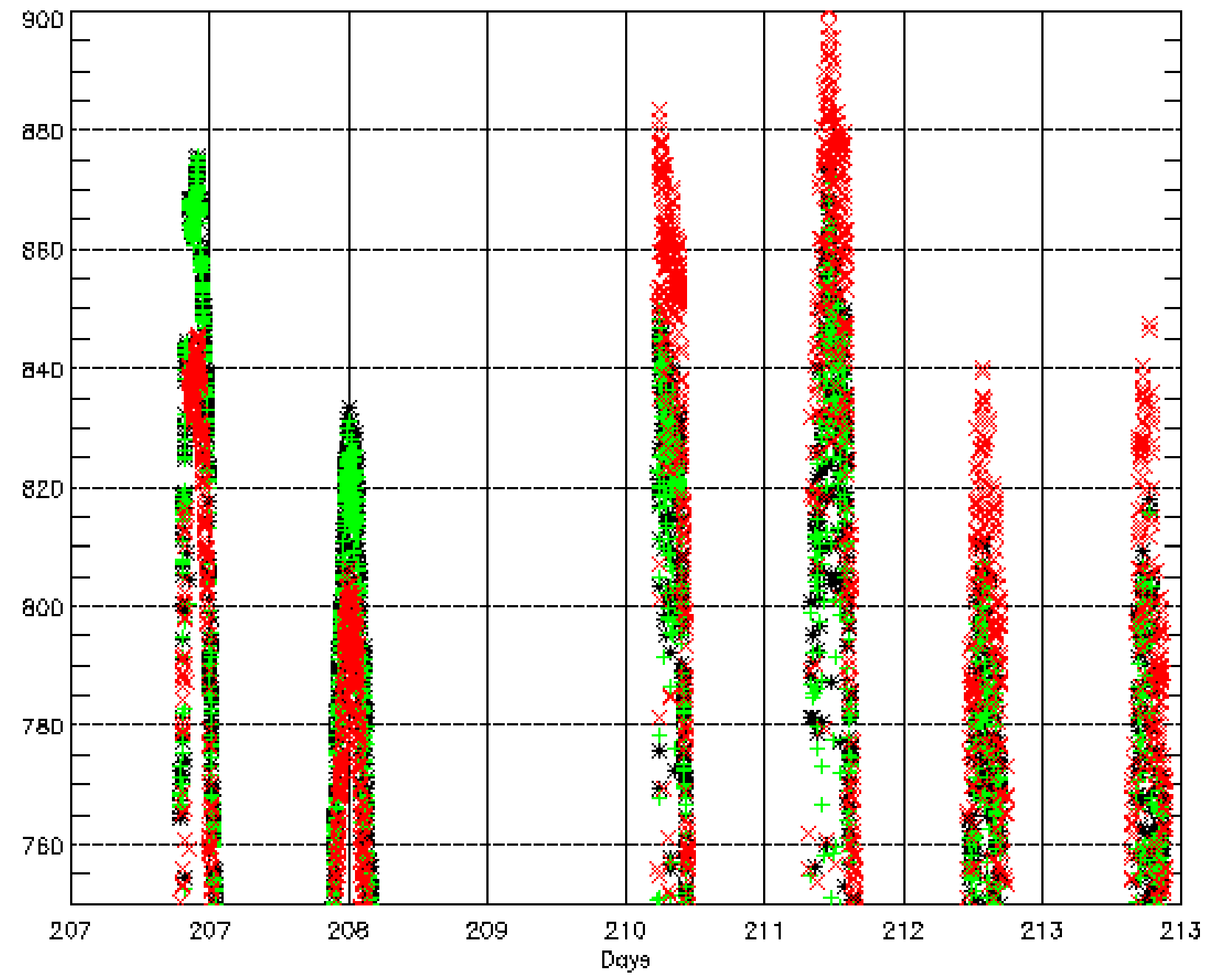

Figure 34. Short Direct Normal data measured at three instruments from day 207 to 213. SIRS E13 (black *), SIRS C1 (green +), and BSRN (red x). 


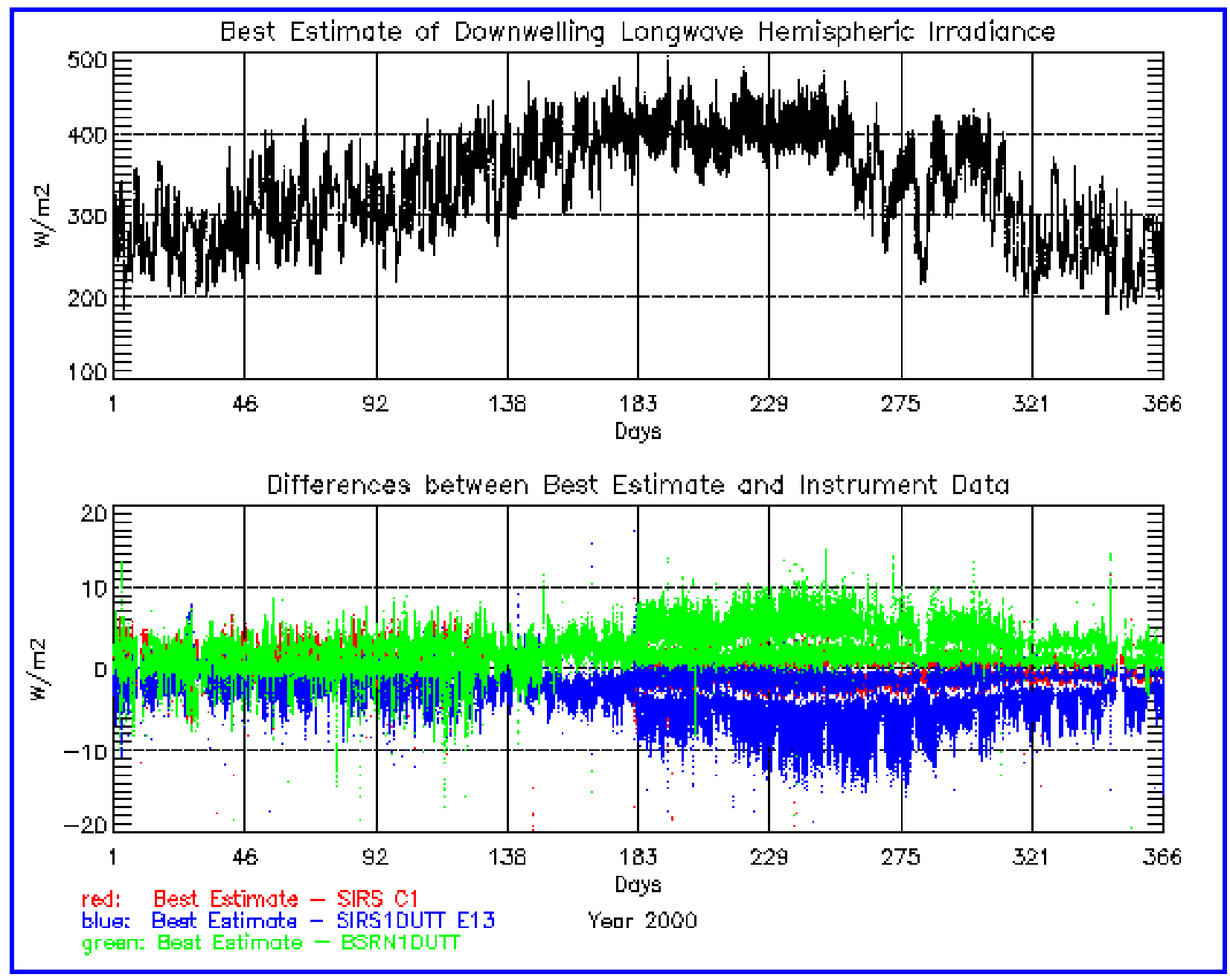

Figure 35. Same as Figure 32, but for downwelling LW. 


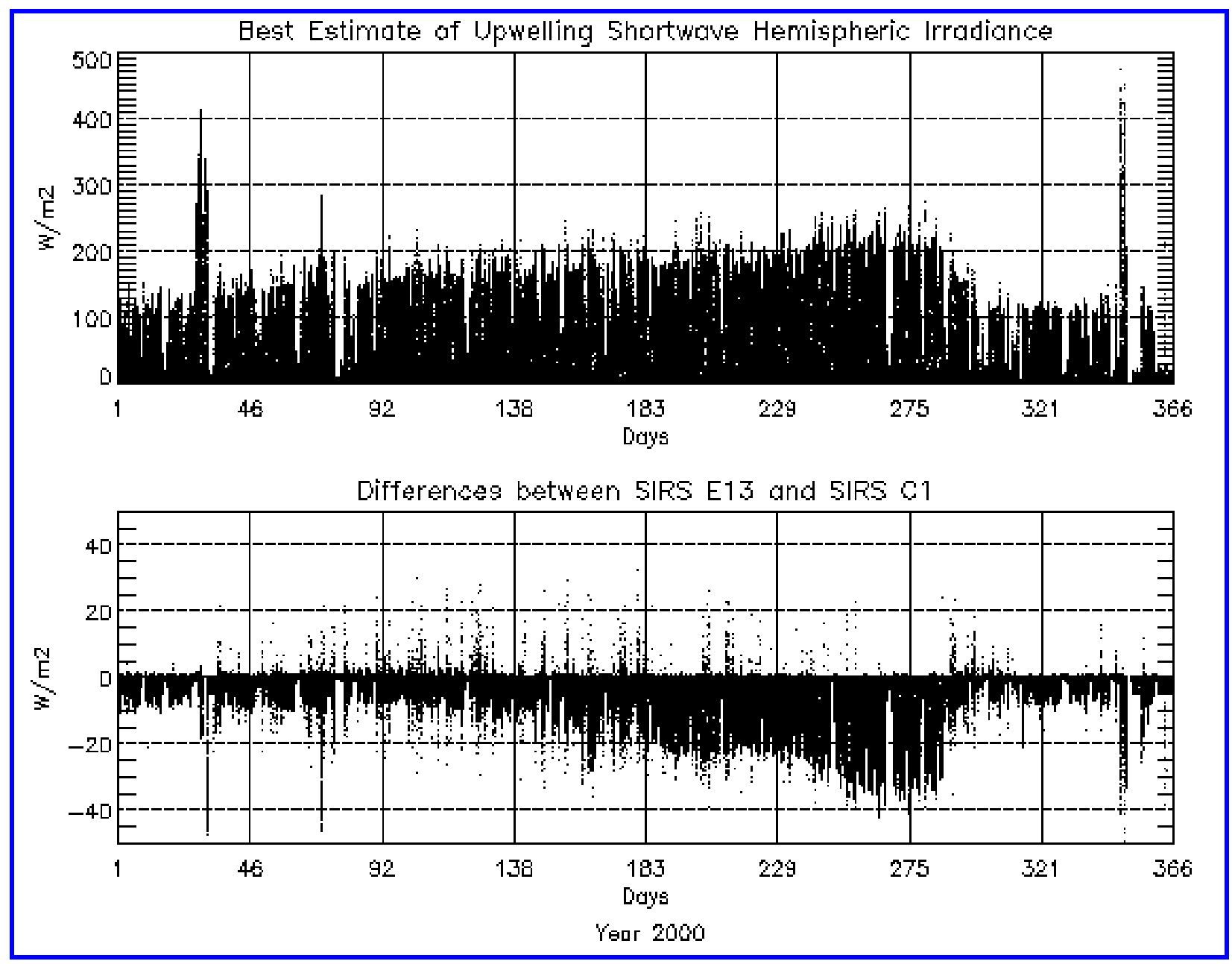

Figure 36. Same as Figure 32, but for upwelling SW. 


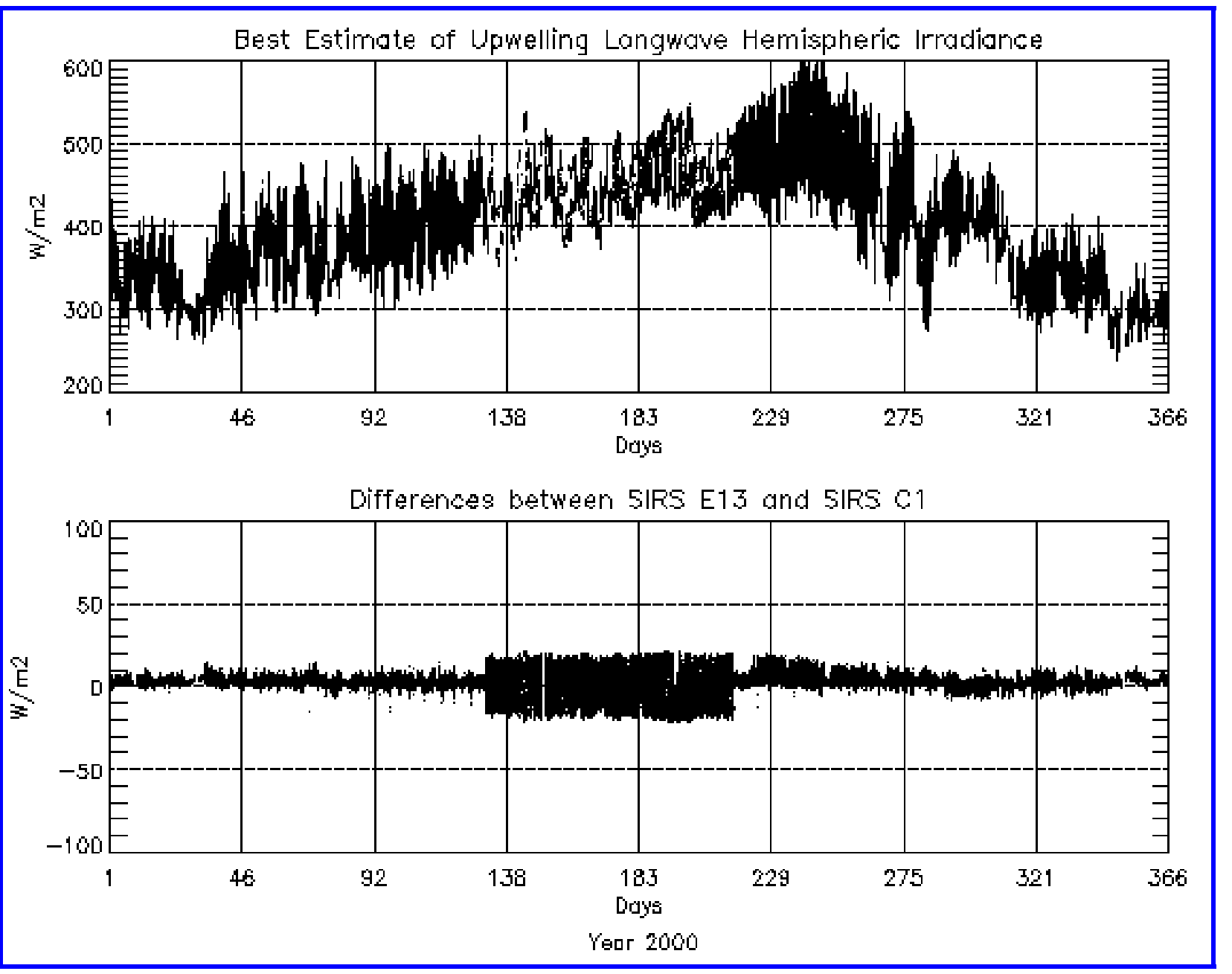

Figure 37. Same as Figure 32, but for downwelling LW.

\section{Analysis: Uncertainty Assessment}

Figure 38 shows the cumulative frequency of instrument differences for diffuse SW in the year 2000. These plots mark where the differences of the two instrument data reach the $95 \%$ level in $\mathrm{Wm}^{-2}$. The top left plot shows the result for all the available data; the top right plot is for QC Flag $=0$, in this case, the best estimate values are obtained by the average of BRS and SIRS E13 data; the bottom left plot is for QC Flag $=1$, when BRS and SIRS C1 data are averaged as the best estimate values; and the bottom right plot is for QC Flag = 2, when the best estimate values are the average of SIRS E13 and C1 data. The red curve in each plot represents the differences between SIRS E13 and SIRS C1; the blue curve represents the differences between BRS and SIRS C1; and the green curve represents the differences between BRS and SIRS E13. Figures 39 and 40 are the same plots as Figure 38, but for direct normal SW and downwelling LW, respectively.

Table 4 is the summary of the 95\% level agreement from 1997 - 2001. The accuracy estimates of Ohmura et al. (Table 3, from Ohmura et al. 1998, BAMS) are based on calibration coefficient data, and primarily represent how well we can calibrate these instruments, or operate them for short periods under 
"ideal” conditions of cloudless skies and low winds. The values in Table 4 represent operational agreement from long-term field deployed instruments, under all-sky and all ambient conditions. When added to the Ohmura et al. values, this represents the "accuracy" we can expect in our Archived data. Our assessment is that "Best" is the best we can do, "Typical" is what one might expect on average for ARM Extended Facilities and BRS data, while "Worst" is what one has to consider as the possible uncertainty of Extended Facility and BRS data.
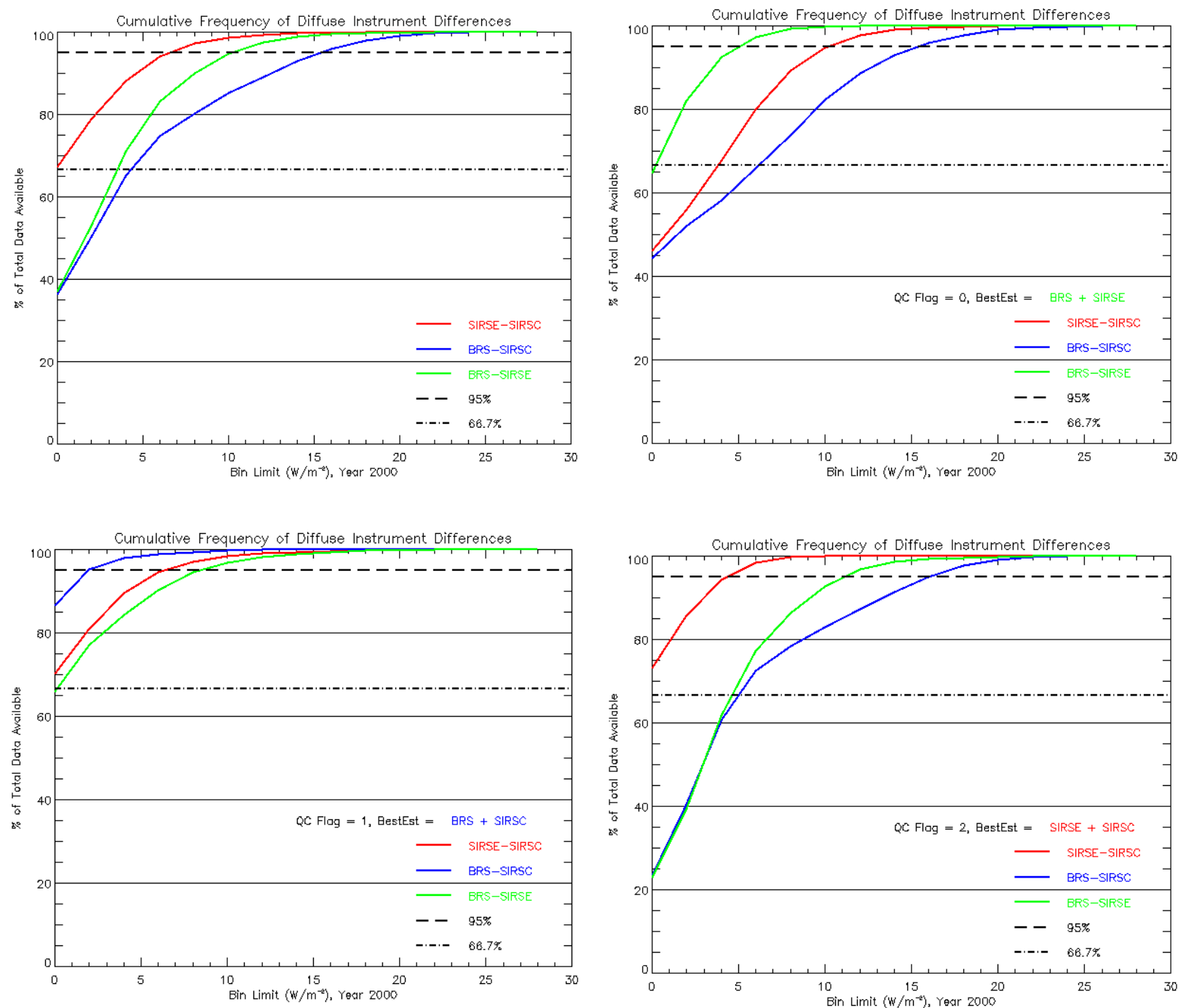

Figure 38. Cumulative frequency of instrument differences for diffuse SW in the year 2000. Top left: all the available data; Top right: QC Flag = 0; Bottom left: QC Flag = 1; Bottom right: QC Flag $=2$. Red: differences between SIRS E13 and SIRS C1; Blue: differences between BRS and SIRS C1; Green: differences between BRS and SIRS E13. 

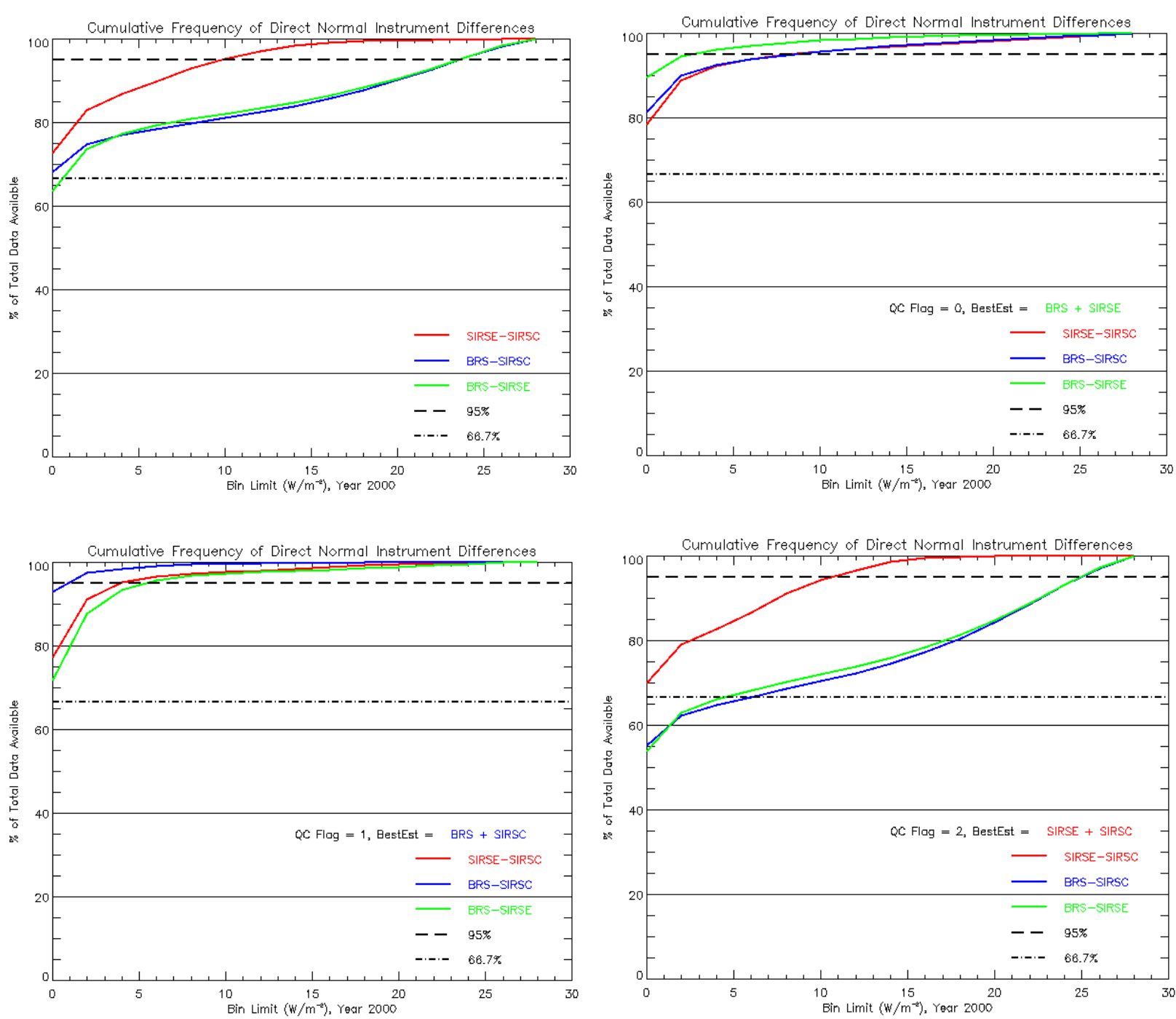

Figure 39. Cumulative frequency of instrument differences for direct normal SW in the year 2000. Top left: all the available data; Top right: QC Flag = 0; Bottom left: QC Flag = 1; Bottom right: QC Flag = 2. Red: differences between SIRS E13 and SIRS C1; Blue: differences between BRS and SIRS C1; Green: differences between BRS and SIRS E13. 

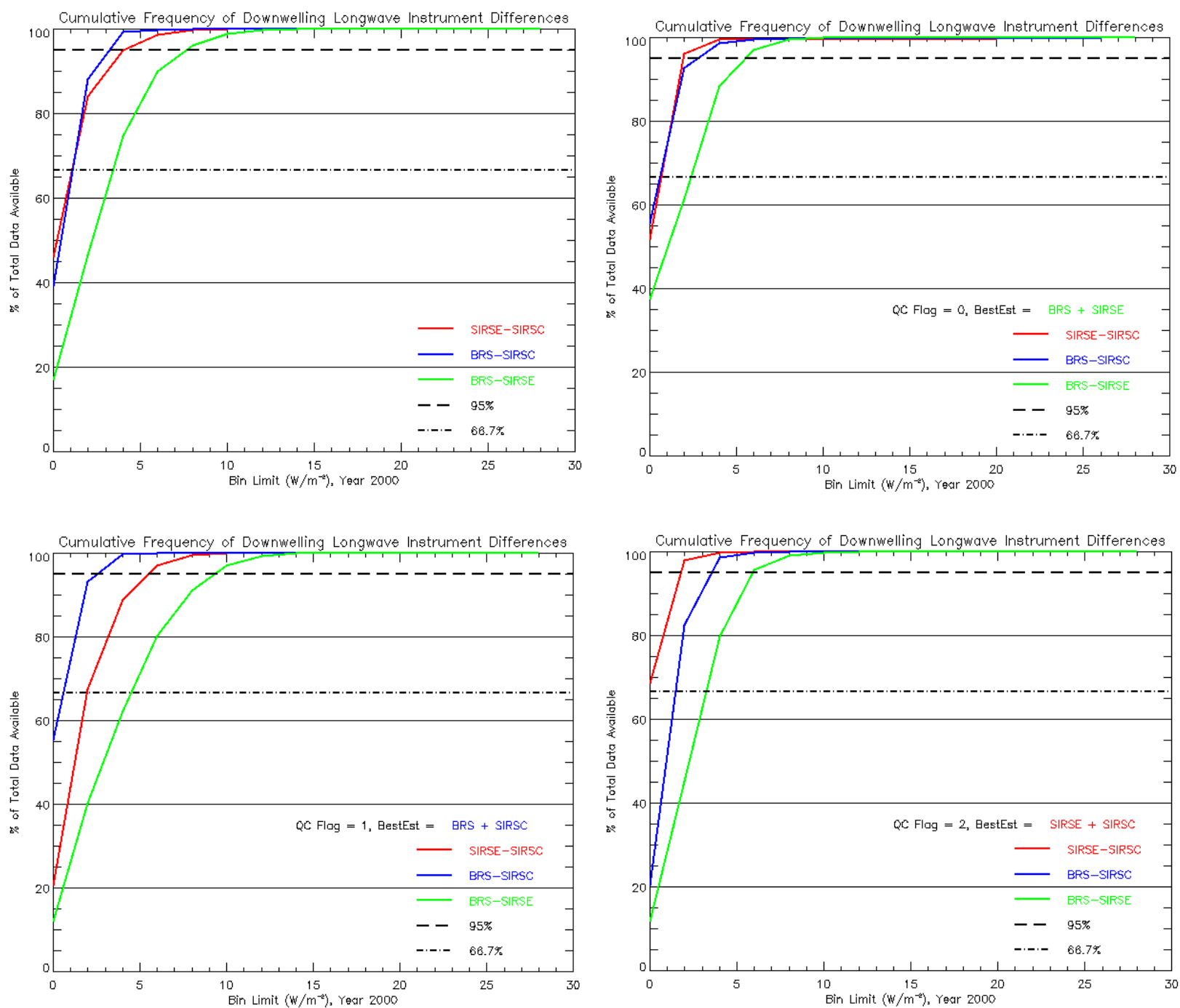

Figure 40. Cumulative frequency of instrument differences for downwelling LW in the year 2000. Top left: all the available data; Top right: QC Flag = 0; Bottom left: $Q C$ Flag $=1$; Bottom right: QC Flag=2. Red: differences between SIRS E13 and SIRS C1; Blue: differences between BRS and SIRS C1; Green: differences between BRS and SIRS E13.

Table 3. From Ohmura et al. (1998, BAMS, Vol. 79, No. 10, pp 2115-2136) Showing Calibration Accuracy

\begin{tabular}{|c|c|c|c|}
\hline \multirow[b]{2}{*}{ Irradiance } & \multirow{2}{*}{$\begin{array}{c}\text { Accuracy requirement } \\
\text { set in } 1990\left(\mathrm{~W} \mathrm{~m} \mathrm{~m}^{-2}\right)\end{array}$} & \multicolumn{2}{|c|}{ Estimated capability $\left(\mathrm{W} \mathrm{m}^{-2}\right)$} \\
\hline & & In 1990 & Achieved by 1995 \\
\hline Global irradiance & 5 & 15 & 5 \\
\hline Direct solar irradiance & 2 & 3 & 2 \\
\hline Diffuse sky irradiance & 5 & 10 & 5 \\
\hline Longwave down irradiance & 20 & 30 & 10 \\
\hline
\end{tabular}


Table 4. Summary of the 95\% Level Agreement from 1997 to 2001

\begin{tabular}{|l|c|c|c|}
\hline & Best & Typical & Worst \\
\hline Diffuse SW & $4.0 \pm 1.4$ & $9.0 \pm 3.1$ & $12.0 \pm 3.8$ \\
\hline Direct Normal SW & $6.3 \pm 3.3$ & $13.6 \pm 6.3$ & $15.0 \pm 6.7$ \\
\hline Downwelling LW & $3.1 \pm 0.4$ & $5.1 \pm 1.2$ & $7.1 \pm 1.4$ \\
\hline Upwelling SW & \multicolumn{3}{|c|}{$11.1 \pm 2.8$} \\
\hline Upwelling LW & \multicolumn{3}{|c|}{$9.6 \pm 3.0$} \\
\hline
\end{tabular}

\section{Summary}

The Best Estimate Flux VAP uses the data available from the three co-located surface radiometer platforms at the SGP CF to automatically determine the best estimate of irradiance values available. The comparative uncertainty of the resulting Best Estimate Fluxes approach the accuracy cited as the best possible for calibrating the instruments themselves by Ohmura et al.

Our analysis during development of the VAP required an assessment of typical agreement between the co-located radiometers in order to determine agreement limits for data QC. The results of this analysis serve as an assessment of the added uncertainty (in addition to the calibration accuracy) of long-term field operated BSRN-type systems, such as the ARM Extended Facilities of the SGP, and the NSA and TWP sites. This "operational uncertainty" is about 4-6 times the values cited by Ohmura et al. for the diffuse and direct normal SW, and about half the Ohmura et al. value cited for the downwelling LW.

\section{References}

Long, C. N., 2002: The ARM Southern Great Plains CF Best Estimate Radiative Flux CD. In Proceedings of the Twelfth Atmospheric Radiation Measurement (ARM) Science Team Meeting, St. Petersburg, Florida, April 8-12, 2002. Atmospheric Radiation Measurement Program Technical Report, ARM TR-007. Available URL: http://www.arm.gov/docs/documents/tech_reports/index.html

Long, C. N., K. Younkin, and D. M. Powell, 2002: Analysis of the Dutton et al. IR Loss Correction Technique Applied to ARM Diffuse SW Measurements. In Proceedings of the Eleventh Atmospheric Radiation Measurement (ARM) Science Team Meeting, Atlanta, Georgia, March 19-23, 2002. Available URL: http://www.arm.gov/docs/documents/technical/conf 0103/long-cn.pdf

Ohmura, A., E. G. Dutton, B. Forgan, C. Frohlich, H. Gilgen, H. Hegner, A. Heimo, G. Konig-Langlo, B. McArthur, G. Müller, R. Phillipona, R. Pinker, C. H. Whitlock, K. Dehne, and M. Wild, 1998: Baseline Surface Radiation Network (BSRN/WCRP): New Precision Radiometry for Climate Research. Bull. of the Amer. Meteo. Soc., 79, No, 10, pp. 2115-2136.

Shi, Y., and C. N. Long, 2002: Techniques and Methods Used to Determine the Best Estimate of Radiation Fluxes at SGP Central Facility. In Proceedings of the Twelfth Atmospheric Radiation Measurement (ARM) Science Team Meeting, St. Petersburg, Florida, April 8-12, 2002. Available URL: http://www.arm.gov/docs/documents/technical/conf_0204/shi-y.pdf 
Younkin, K., and C. N. Long, 2002: Results of the Dutton at al. IR Loss Correction VAP: Statistical Analysis of Corrected and Uncorrected SW Measurements. In Proceedings of the Twelfth Atmospheric Radiation Measurement (ARM) Science Team Meeting, St. Petersburg, Florida, April 8-12, 2002.

Available URL: http://www.arm.gov/docs/documents/technical/conf_0204/younkin-k.pdf 